\title{
Monographs on Physics
}

\section{SLRA}

\section{MODERN SEISMOLOGY}

\author{
G.W.WALKER, R, R,
}







\section{MONOGRAPHS ON PHYSICS}

EDITED BY

Sir J. J. THOMSON, O.M., F.R.S.

AND

FRANK HORTON, D.Sc., M.A.

OF THE CAVENDISH LABORATORY, CAMBRIDGE 


\title{
MONOGRAPHS ON PHYSICS
}

EDITED BY

\author{
SIR J. J. THOMSON, O.M., F.R.S. \\ AND \\ FRANK HORTON, D.Sc., M.A. \\ of the Cavendish Laboratory, Cambridge
}

8vo.

RAYS OF POSITIVE ELECTRICITY AND THEIR APPLICATION TO CHEMICAL ANALYSIS. By Sir J. J. Thomson, O.M., F.R.S. 5s. net. [In the press.

MODERN SEISMology. By G. W. Walker, A.R.C.Sc., M.A., F.R.S., formerly Fellow of Trinity College, Cambridge. With Plates and Diagrams. 5s. net.

PHOTO-ELECTRICITY, THE LIBERATION OF ELECTRONS BY LIGHT; with Chapters on Fluorescence and Phosphorescence, and Photo-Chemical Actions and Photography. By H. Stanley Allen, M.A., D.Sc., Senior Lecturer in Physics at University of London, King's College. 7s. 6d. net.

THE SPECTROSCOPY OF THE EXTREME ULTRAViOLET. By Professor Theodore Lyman, Jefferson Physical Laboratory, Harvard University, Cambridge, Mass.

[In preparation.

COLloidAl SOLUTIONS. By Professor E. F. Burton,

The Physics Department, The University, Toronto.

[In preparation.

ATMOSPHERIC IONIZATION. By Professor J. C. McLennan, Department of Physics, The University, Toronto.

[In preparation.

THE EMISSION OF ELECTRICITY FROM HOT BODIES. By O. W. Richardson, F.R.S., Wheatstone, Professor of Physics, King's College, London.

[In preparation.

Electric Waves. By Professor G. W. Pierce, Harvard University, Cambridge, Mass.

[In preparation.

LONGMANS, GREEN AND CO. 39 PATERNOSTER ROW, LONDON NEW YORK, BOMBAY AND CALCUTTA 



\section{PREFATORY NOTE.}

ONLY a week after this book had been handed to the publisher, the scientific world had to mourn the loss of Dr. John Milne, who entered into Rest on 3 I July, I9I3.

It was my melancholy privilege on 5 August to pay a last tribute to one who had proved a very kind friend.

The assembling of a large congregation in St. Thomas's Church, Newport, Isle of Wight, was an eloquent testimony to the love and esteem with which Milne was regarded by those among whom his daily life was spent.

No one will deny that Milne was truly the father of modern Seismology. He founded the subject, he developed it well-nigh single-handed, and he lived to see the importance of his life work recognised not only by his fellow-countrymen but by the whole civilized world.

The credit for several important points in modern Seismology is sometimes assigned to others, and it was only Milne's greatness of heart that prevented him from claiming the priority that was rightly his.

But his claim to scientific fame rests not on details, for he made the whole subject. As Prince Galitzin remarked at Cambridge only a year ago, "There are not many questions of modern Seismology that have not been attacked by Milne long before any other person had thought about them".

G. W. W. 


\section{CONTENTS.}

\section{SEISMOMETRY.}

CHAP.

I. General Dynamical Theory of Seismographs . . . .

II. Methods of attaining Sensitiveness, Damping, Registration . 7

III. Chief Types of Seismographs in Actual Use. Milne, Omori, Bosch, Wiechert, Galitzin . . . . . • . I6

IV. Standardization of Seismographs, including Theory of Mechanical and Electromagnetic Registration. . . 2 I

V. Installation of Seismographs and Consideration of Develop-

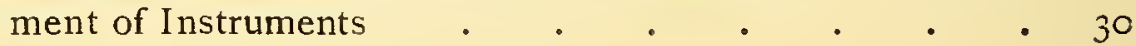

\section{SEISMOGEOPHYSICS.}

VI. Theory of a Solid Isotropic Earth . . . . . 37

VII. Interpretation of Seismograms and the Interior of the Earth . 48

VIII. Determination of Epicentre and Focus . . . . . . 63

IX. Seismic Effects other than those due to Earthquakes . $\quad 72$

X. Statistical . . . . . . . . . . 83 


\section{LIST OF PLATES}

PLATE

I. Milne Horizontal Seismograph for one Horizontal Component . . . . . facingpage 16

2. Wiechert Astatic Inverted Pendulum Seismograph for two Horizontal Components . , , , I7

3. Galitzin Aperiodic Horizontal Component Seismograph, with Galvanometer and Recording Cylinder for electromagnetic registration .

4. Galitzin Aperiodic Vertical Component Seismograph for electromagnetic registration

5. Time Curves (after Zöppritz) . . . . . . page

6. Showing Maximum Depth of Seismic Rays as Function of Epicentral Distance (after Zöppritz) . • . • . • . " "

7A. Portion of Record at Pulkowa, September I8, I9I0, showing microseismic movement . facing page

7B. Specimen Record (reduced) showing Tilt produced by Tidal loading . . . . " " "

8. Earthquake in Yap, West Caroline Islands. Milne Seismogram, Eskdalemuir, August I6-I7, I9II. N-S Component

9. Earthquake in Yap, West Caroline Islands. Milne Seismogram, Eskdalemuir, August I6-I7, I9II. E-W Component . . " , "

Io. Specimen Record-Wiechert Seismograph ( $1200 \mathrm{~kg}$.) with ink registration . . , ",

II. Specimen Record-Galitzin Horizontal Component Seismograph . . . .

12. Specimen Record-Galitzin Horizontal Component Seismograph

13. Specimen Record-Galitzin Vertical Component Seismograph . . . . . 


\section{INTRODUCTION.}

THE present volume owes its existence to Sir J. J. Thomson's suggestion that I should write an account of the present position of seismological investigation.

The book is written from the point of view of Seismology as a branch of Physics, and particularly as it is determined by observatory conditions. My qualification to deal with the subject in this aspect rests on what is probably the unique experience of having set up at Eskdalemuir and having had under daily personal observation a Milne twin-boom seismograph, a Wiechert $80 \mathrm{kgm}$. two horizontal components astatic inverted pendulum seismograph, a complete Galitzin installation of seismographs with galvanometric registration for three components, and an Omori seismograph for one horizontal component. Simultaneous records of the magnetographs and autographic meteorological instruments were also available for comparative study.

This limited treatment of the subject is determined as much by conditions of available space, as by my ignorance of the geological side and of the practical application of earthquake study to building construction which is of so much importance to those who live under the daily danger of the "earthquake". But the limitation is no disadvantage since we already have Dr. Milne's works on "Earthquakes" and "Seismology" (International Science Series), which deal with the subject in its wider aspects and with the authority of Milne's unrivalled personal experience.

The history of Seismology has been traced back to the viii 
earliest times. It would be interesting to know whether the ancients possessed any accurate knowledge of the subject, as they did in the case of Astronomy.

The literature of the subject is very extensive, but until comparatively recent years it contains much that is speculative, much that is inaccurate, and much that is false, as is ever the case with a science until it becomes quantitative. One recalls Lord Kelvin's first criterion of knowledge of a phenomenon, viz., "How much of it is there?"

If the newest literature is not entirely free from speculation and inaccuracy, the study now proceeds on a quantitative basis which enables the reader to judge for himself as to the value of the conclusions.

The new Seismology as a quantitative physical science may be said to have started about thirty years ago, and with a small band of British scientists in Japan. These pioneers were Milne, Gray, Ewing, Perry and Knott.

Germany and Italy may also claim pioneers about the same time.

The horizontal pendulum adopted by Milne appears to have been independently invented in slightly different forms by different investigators, and it is difficult to assign priority. The horizontal pendulum in the forms used by Zöllner and Rebeur-Paschwitz deserve special mention.

The experimental discovery that an earthquake could be registered by a delicate pendulum at great distances from the focus marks the first step in the new science. It is undoubtedly to Milne's vigorous personality that we owe the application of this fact to the study of earthquakes. On his return to this country in 1895 he set up his observatory at Shide in the Isle of Wight, and by the installation of his instruments at a number of stations distributed all over the earth, he inaugurated the first Seismological Service. Comparative data were thus obtained, and rapidly led to an increased knowledge of the properties of the earth. 
That the seismogram of a distant earthquake represents elastic waves that have travelled through the earth from focus to station was early recognised, but the well-known result that a solid body transmits longitudinal and transversal waves with different speeds did not at first seem to throw much light on the meaning of the seismogram, which by its apparent complexity suggested a highly heterogeneous earth.

It was, however, an important thing for seismological theory when Lord Rayleigh (see "Collected Papers") showed in I 885 that there could be propagated along the surface of an elastic solid a set of waves travelling with speed rather less than that for transversal waves. Such waves play an important part in the long wave phase of a seismogram which develops some time after the first indication of a disturbance. Milne applied this in I 895 to show that the interval of time between the apparent "start" and the occurrence of the long waves on the record provided a means of estimating the distance of the epicentre. Although the estimate is not very accurate, it was really the first step in the interpretation of seismograms and in the location of the epicentre from observations made at distant points.

About this time we owe to Rudski ("Physik der Erde") and to von Kövesligethy ("Seismonomia") theoretical investigations of the path of seismic rays within the earth. The first application of the well-known theory of longitudinal and transversal waves to Milne seismograms appears to have been made by Oldham ("Phil. Trans. R.S.," I 900). Milne seismograms, however, partly because of the presence of instrumental vibration and partly because of the comparatively slow speed of registration, do not readily lend themselves to an accurate $a$ priori estimate of the occurrence of the second or transverse phase. Thus no great progress to accuracy seems to have been made until the interpretation of seismograms was taken up by Wiechert and his pupils at Göttingen.

Wiechert's investigations began about I900, when, at the 
request of the German Government, he made a tour to the Italian observatories, and as a result of his studies designed and set up the inverted astatic pendulum now known by his name. By the introduction of fairly large damping, of increased magnification, and of increased speed of registration, the accuracy was greatly increased, and the division of a seismogram into three principal phases corresponding to the longitudinal, transversal, and long waves became a comparatively simple and definite process.

In I903 Wiechert published a most important memoir on the "Theory of Automatic Seismographs" ("Abhand. der König. Gesell. der Wiss. Göttingen") showing, among other things, the quantitative relation of the recorded movement of the instrument to that impressed on the pier. This memoir was followed in 1907 by a paper on "Earthquake Waves" by Wiechert and Zöppritz ("Gött. Nachrichten," I907). On the experimental side greatly improved time curves giving the time of arrival of the longitudinal and transversal waves as functions of the epicentral distance are obtained. The results have been used by Zeissig in the preparation of his interpolated tables giving the epicentral distance for the time interval between the arrival of the two sets of waves now known as $\mathrm{P}$ and $\mathrm{S}$. These tables (published by the Imper. Academy of Sciences, St. Petersburg) are now in general use and are the most accurate we have. On the theoretical side Wiechert adds greatly to the interpretation of seismograms, and shows how the time curves lead to a knowledge of the physical properties of the interior of the earth. Wiechert and his pupils are still actively engaged in the extension of our knowledge in this direction.

Galitzin's investigations began about the same time as those of Wiechert and have proceeded on somewhat different lines. It may be said that the problem he set himself was to make instrumental seismometry a truly quantitative art as 
measured by the standard of modern experimental physics in the laboratory.

He was led to adopt electromagnetic damping up to the limit of aperiodicity and to introduce electromagnetic registration to get increased magnification. Each point of construction or of theory was submitted to the most rigorous tests in the physical laboratory until success was attained, and the observatory of Pulkowa started continuous recording and publication of observations on I January, I 9 I 2.

His separate memoirs have appeared in the "C.R. of the Imperial Academy of Sciences, St. Petersburg," and the results are embodied in his book published last year ("Lectures on Seismometry"). The whole investigation is a most instructive and masterly application of physical principles to observational seismometry.

Perhaps the most striking result attained by Galitzin is a complete experimental proof that his instruments determine not only the distance of the epicentre, but also the azimuth from the observing station, so that it is now possible from observations at a single station to determine the epicentre within the limits that must be assigned to the epicentral region itself.

Seismographs reveal the existence of earth movements other than those due to earthquakes. Chief of these are the movements known as microseisms and earth-tides. Seismology is thus brought into intimate connexion with Astronomy and Geodesy.

It may truly be claimed that during the close of the nineteenth and the beginning of the twentieth century seismologists, among whom the names of Milne, Wiechert and Galitzin stand pre-eminent, have succeeded in dragging the study of earthquakes from the region of ignorance and superstition and in making it a quantitative science proceeding on the principles of physical philosophy. 


\section{ERRATA.}

P. 3, Equation B, read " $-\ddot{x} / l "$.

P. 43, line 17 , for " $\tan \vec{e}=-\cos 2 \ell^{\prime} "$ read $" \tan \bar{c}=-\cot 2 e^{\prime}$ ".

P. 44 , line 23 , for " $t$ "read " $\mathrm{T}$ ".

P. 47 , line 2 , read "increasing to $\pi / 2$ for $\theta=\pi$ ".

P. 5\%, line 8 , for "E" read " $e$ ".

P. 65, line 3I, for "August "read "September".

P. 69, line 22, after " $400 \mathrm{~km}$." introduce "it". 



\section{CHAPTER I.}

GENERAL DYNAMICAL THEORY OF SEISMOGRAPHS.

THE most general movement of the ground in the vicinity of a point on the earth's surface may be regarded as made up of the components of a linear displacement resolved along three mutually perpendicular axes and the components of a rotation resolved about these three axes. It is convenient to choose the geographical axes at the point, viz. North, East, and Vertical.

In practical seismometry the horizontal components have been mainly the subject of measurement, and it is but recently that the vertical movement has been carefully studied. The rotations are not at present recorded, although experiments directed to that end are now in progress at Pulkowa. The principal seismic waves recorded are, however, many kilometres in wave length, while the amplitude at some distance from even a devastating earthquake is but a fraction of a millimetre, so that the twisting movement is practically small except in the vicinity of the earthquake, where actual measurements are for obvious reasons of a rough and hazardous nature.

Thus the objective of a seismological station being primarily the recording of the earth movement experienced there, we have to consider the instruments by means of which records are obtained.

The instruments are called seismographs, and each seismograph measures, or is supposed to measure, one component. Thus six instruments are theoretically required to determine the complete motion, but at present only a few stations are fitted with three seismographs for the three linear components, while most stations possess only two instruments for recording the two horizontal components. 
Any stable dynamical system which is set into relative movement when its supporting platform is moved may be called a seismometer, because it is purely a matter of dynamics to determine the quantitative relation between the observed movement of the instrument and the motion of the platform, which is also that of the ground and is the object of investigation.

The simplest seismometer for horizontal

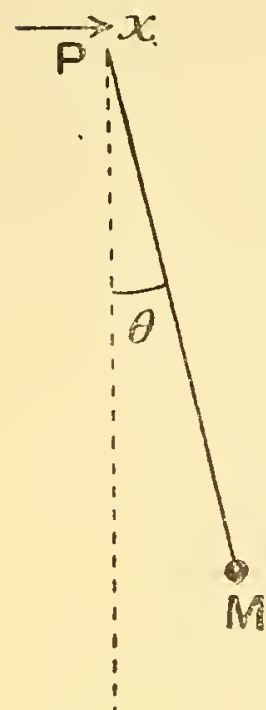

FIG. I. motion is a simple pendulum supported from a point on a rigid framework fixed to the ground. We may imagine that a pencil fixed to the bob writes on a sheet of paper held horizontally beneath it, so as to give a record of the movement experienced by the bob when the earth moves. This simple apparatus would register on a small scale the horizontal components of the earth movement; and since the equation of motion is in form precisely that which applies to any seismograph, we shall do well to examine it before proceeding to consideration of seismographs actually used.

Let $l=$ length of string,

$g=$ acceleration of gravity,

$y=$ displacement of bob horizontally,

$x=$ prescribed horizontal motion of $\mathrm{P}$ the point of support ;

then for small motions we have

$$
\ddot{y}+(y-x) g / l=0
$$

as the equation of motion. (Rayleigh "Theory of Sound," p. 63.)

Now the movement registered is not $y$ but $y-x$ since the paper must also be supposed to have the motion $x$.

Hence if $\xi=y-x$ the equation becomes

$$
\ddot{\xi}+\xi g / l=-\ddot{x} .
$$

If $\theta$ be the angular displacement of the string we have $\theta=\xi / l$ and hence

$$
\ddot{\theta}+g \theta / l=-\ddot{x} / l
$$

The distinction between the actual movement of the bob and the movement recorded is important, as in all seismographs the 
registering apparatus, etc., must participate in the earth movement $x$ and thus it is the relative movement that is registered.

The equation may be obtained otherwise by superposing on the whole system the reversed earth movement and then taking moments about $\mathrm{P}$ now regarded as a fixed point.

We should get an equation of the same form as (A) for a compound pendulum, $l$ being now the length of the equivalent simple pendulum.

The simple pendulum has another feature in common with all horizontal component seismographs, namely, that it records not only linear horizontal displacement of the ground but also rotation about a horizontal axis. Thus if $\psi$ represents the angular displacement of platform, etc., about. an axis through $\mathrm{P}$ perpendicular to the plane of the paper measured positive in the clock-ivise direction the equation becomes

$$
\ddot{\theta}+\theta g / l=-\ddot{x} / l+\psi g / l+\ddot{\psi}
$$

where $\theta$ is now the apparent angular displacement of the string. We may incorporate $\ddot{\psi}$ with $\ddot{x}$, if the latter is now regarded as the horizontal linear acceleration that would be experienced by a point coinciding with the nul position of $M I$ and rigidly connected to the earth, while the axis of rotation of $\psi$ is moved to $M$. As has already been stated the rotation is, in the case of an earthquake at some distance, so small that the seismograph is usually regarded as measuring solely the linear motion.

All vibrating systems are subject to frictional forces and we must now introduce the necessary modification of the fundamental equation on this account. The assumption is usually made that the frictional forces can be represented by a term proportional to the angular velocity $\dot{\theta}$. The mathematical convenience of the assumption is enormous, and in some cases the assumption is in sufficiently good agreement with fact.

The equation then takes the form

$$
\ddot{\theta}+2 \epsilon \dot{\theta}+n^{2} \theta=\quad \ddot{x} / l \text {. }
$$

and this is the fundamental equation in instrumental seismometry.

Wiechert has remarked ("Theory of Autographic Seismographs," "Abhand, Kön. Gesell. d. Wiss.," Göttingen, 1903), 
that all seismographs are fundamentally the same, and if the frictional term could always be expressed as above no objection could be taken to the statement. The different behaviour of instruments in actual practice is, however, mainly due to the fact that the frictional term is not of this simple form in all cases.

The equation (B) is of well-known form, and full treatment may be found in any treatise on differential equations (e.g. Forsyth).

The free motion is given by

$$
\ddot{\theta}+2 \epsilon \dot{\theta}+n^{2} \theta=0
$$

and the solution of this is of the form

$$
\begin{array}{ll}
\theta=\mathrm{A} e^{-\epsilon t} \sin \left\{\left(n^{2}-\epsilon^{2}\right)^{\frac{1}{2}}(t-\eta)\right\} & \text { for } n>\epsilon \\
\theta=\mathrm{A} e^{-\epsilon t} \sinh \left\{\left(\epsilon^{2}-n^{2}\right)^{\frac{1}{2}}(t-\eta)\right\} & \text { for } n<\epsilon \\
\theta=\mathrm{A} e^{-\epsilon t}(t-\eta) & \text { for } n=\epsilon
\end{array}
$$

where $\mathrm{A}$ and $\eta$ are arbitrary constants.

The last case is of special importance in modern seismometry, and the instrument is then described as "dead-beat" or "aperiodic".

In any case the quantities $n$ and $\epsilon$ are instrumental constants which may be determined experimentally by methods well recognized in ordinary laboratory practice. The quantity $x$ is in general a function of time and the recorded movement $\theta$ then consists of two parts: (I) depending on the special form of $x$, and (2) depending on the free movement of the instrument with constants depending on the initial conditions. The complete solution when $x$ is any prescribed function is given by Rayleigh ("Theory of Sound," p. 74).

We shall consider only the case in which $x$ is a simple periodic function of the time, say $x_{0} \sin (p t)$.

The forced movement $\theta$ corresponding to this is

$$
\theta=\frac{p^{2} x_{0} \sin (p t-\eta)}{l\left\{\left(n^{2}-p^{2}\right)^{2}+4 \epsilon^{2} p^{2}\right\}^{\frac{1}{2}}} \text { where tan } \eta=\frac{2 \epsilon p}{n^{2}-p^{2}} .
$$

The recorded movement thus differs in phase from the impressed movement. As the actual recorded quantity will be proportional to $\theta$ say $\mathrm{L} \theta$

the expression 


$$
\frac{\mathrm{L}}{l} p^{2}\left\{\left(n^{2}-p^{2}\right)^{2}+4 \epsilon^{2} p^{2}\right\}^{-\frac{1}{2}}
$$

represents the "magnification" of the amplitude of the earth movement.

Terms representing the free motion will also appear on the record, and these have a diminishing amplitude. Now the practical problem is to determine the earth movement $x$ from the recorded movement so that even this simple case shows us how important it is that the "free" terms should be made to subside with rapidity. A fortiori it is evident that if $x$ is undergoing complicated changes, it is difficult to form any true conception of the earth movement from the seismogram unless the "free" terms are made to subside quickly. Thus the necessity for a large value of $\epsilon$, that is very great damping, becomes apparent.

The expression for the magnification may be written

$$
\mathrm{M}=\frac{\mathrm{L}}{l} \cdot \frac{\mathrm{I}}{\mathrm{U}}
$$

where $U=\left\{\left(u^{2}-\mathrm{I}\right)^{2}+4 u^{2} \epsilon^{2} / n^{2}\right\}^{\frac{1}{2}}$ and $u=n / p$.

Thus $\mathrm{U}$ is unity when $p=\infty$ that is for infinitely rapid vibrations, while $\mathrm{U}$ is infinite when $p=0$. Thus the magnification is nil for infinitely slow vibrations.

$U$ clearly becomes a minimum for different values of $u$ when

$$
u^{2}=\mathrm{I}-2 \epsilon^{2} / n^{2}
$$

and we may choose $\epsilon / n$ so as to get the minimum for any prescribed values of $u$.

If $\varepsilon / n=\mathrm{I} / 2^{\frac{1}{2}}$ we get $u=0$ as the minimum, and this value has the advantage of making the magnification for rapid waves more nearly constant for different periods than would otherwise be the case.

If the instrument is aperiodic $\epsilon / n=I$ and there is then a minimum at $u=0$ for $\mathrm{U}$, which now takes the form $\left(u^{2}+\mathrm{I}\right)$. In certain theoretical investigations it is convenient to use quantities related to $n$ and $\epsilon$ as follows :-

$$
h=\epsilon / n, \mu^{2}=\mathrm{I}-h^{2} \text { and } \gamma=\left(n^{2}-\epsilon^{2}\right)^{\frac{1}{2}}=\mu n
$$

so that $h=\mathrm{I}$ or $\mu^{2}=0$ expresses the condition for aperiodicity. 
The simplest seismometer for vertical motion is a small mass suspended by a light elastic string so that vibration may take place vertically.

It is unnecessary to prove here that the equation of motion takes precisely the form we have already considered, so that the expressions already obtained are equally applicable to seismographs for measuring horizontal or vertical motion. 


\section{CHAPTER II.}

\section{METHODS OF ATTAINING SENSITIVENESS, DAMPING, REGISTRATION.}

IN general the amplitude of the earth movement to be measured at a seismological station is small, the convenient unit being $\frac{1}{1000}$ th $\mathrm{mm}$. or micron. Thus a seismograph to be of practical value must give a large magnification of the earth movement.

The expression for the magnification

$$
\begin{aligned}
\mathrm{M} & =\frac{\mathrm{L}}{\mathrm{l}} \cdot \frac{\mathrm{I}}{\mathrm{U}} \\
\text { where } \mathrm{U} & =\left\{\left(u^{2}-\mathrm{I}\right)^{2}+4 \epsilon^{2} u^{2} / n^{2}\right\}^{\frac{1}{2}} \text { and } u=n / p
\end{aligned}
$$

shows that it depends on the indicating length $\mathrm{L}$. It will be convenient to consider $\mathrm{L}$ in connexion with registration and for the present to treat it as prescribed.

For very rapid vibrations $U$ equals $I$ and thus we should gain by making $l$ small. I do not think the question of the best dimensions for a pendulum has received much attention, but it deserves consideration.

For very slow waves we find that we gain by making $n^{2} l$ small. Now $n^{2}=g / l$ and so $n^{2} l=g$ and thus the dimensions are without influence. Thus attention has been directed to making what we may call the static sensitiveness great, by reducing the effective control.

It is possible to reduce the influence of gravity in controlling a compound pendulum. If the axis of rotation is gradually altered from the horizontal to the vertical direction the effective part of gravity in controlling rotation of the body about that axis becomes less and less, until when the axis is vertical the influence is nil. This is the principle on which horizontal pendulums are constructed. The name horizontal 
pendulum is used because the length of the pendulum lies nearly along a horizontal axis, not because the pendulum measures the horizontal component of motion.

It is not possible to make the axis of rotation strictly vertical as a practical limit is reached at which the instrument becomes unstable. This depends on the limit of accuracy of mechanical workmanship.

The arrangement is represented in the diagram (fig. 2) where $\mathrm{BV}$ is the vertical, $\mathrm{AB}$ the axis of rotation making an angle $i$ with $\mathrm{BV}$, while $\mathrm{CM}$ is the rod and mass.

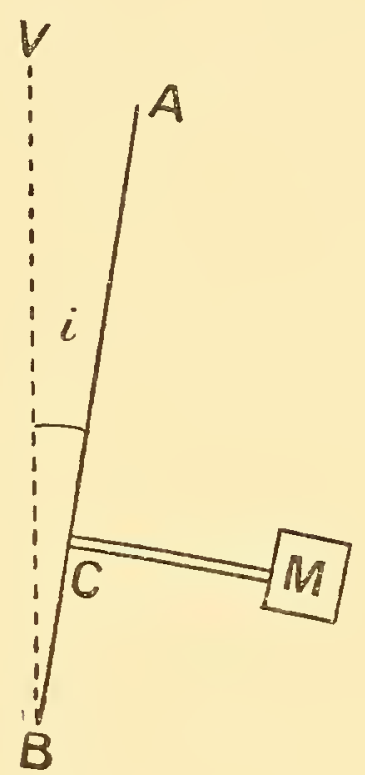

FIG. 2.

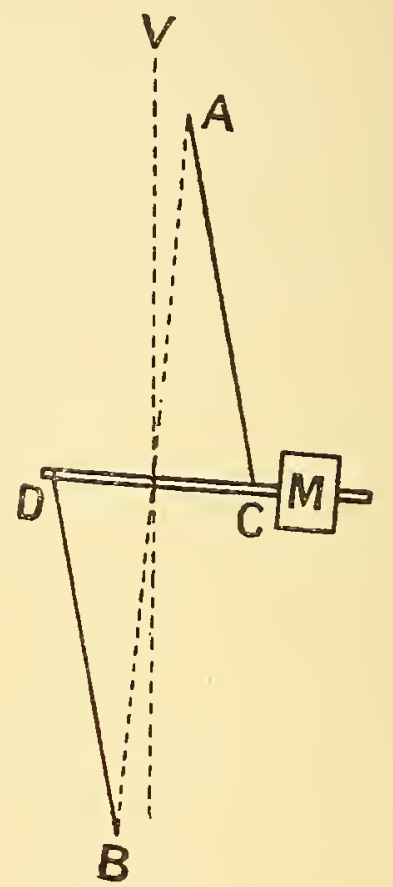

FIG. 3.

The line $A B$ may be actually a rigid rod pivoted at fixed points $A$ and $B$ about which the rotation takes place. The instruments of Hecker and Rebeur-Paschwitz are of this type. But we can also make $C$ a fixed pivot and support $M$ by a string or wire to a fixed point $A$, and $A B$ is then an ideal line about which the system turns. Types of these are the Milne, Bosch, and Omori Seismographs. Pivots are liable to become defective, and in any case introduce frictional effect that cannot be adequately considered theoretically, so that flat steel springs replace the pivot $\mathrm{C}$ in the pendulums of Mainka and Galitzin pendulums for second order stations.

Another form of suspension that possesses great advantages 
is due to Zöllner and is shown in the diagram (fig. 3). The pendulum rod $C D$ is supported by wires $A C$ and $B D$ both under tension on account of the mass $\mathrm{M}$, and clamped to fixed points $A$ and $B$ so that $A B$ is the axis of rotation.

This method is used by Galitzin in his aperiodic pendulums. In all these cases the angle $i$ is practically very small, and clearly we may regard the system as a compound pendulum controlled by "reduced gravity" of amount of $g \sin i$ or $g i$.

In this way periods of 20 seconds or more can readily be attained implying a large increase of magnification.

If $M k^{2}$ represents the moment of inertia of the system about $\mathrm{AB}$,

$$
h=\text { distance of the C.G. from AB }
$$

and $\theta$ in the apparent angular motion, we get the equation

$$
\mathrm{M} k^{2} \ddot{\theta}+\mathrm{Mg} h i \theta=-\mathrm{M} h \ddot{x}
$$

while if we introduce a frictional term proportional to $\dot{\theta}$ we may reduce the equation to the form

$$
\ddot{\theta}+2 \epsilon \dot{\theta}+n^{2} \theta=-\ddot{x} / l
$$

wherein $n^{2}=g h i / k^{2}$, and $l=k^{2} / h$ is as before the length of the equivalent simple pendulum. CM the axis of the pendulum is very nearly horizontal, and we have to observe that the pendulum will record not only horizontal motion of the ground represented by $x$ but also tilting represented by rotation $\psi$ about a horizontal axis coinciding with $\mathrm{CM}$ and rotation represented by $\chi$ about a vertical axis.

It is easy to show that the complete equation is

$$
\ddot{\theta}+2 \epsilon \dot{\theta}+n^{2} \theta=-\ddot{x} / l+g \psi / l+\ddot{\chi}(l-h) / l
$$

but in obtaining the equation it is important to remember precisely what the quantities are, viz. :-

$\theta$ is the apparent angular movement.

$\ddot{x}$ is the acceleration that would have been experienced by a point coinciding with the C.G. of the pendulum but rigidly attached to the earth.

$\psi$ is the angular rotation about the horizontal axis through the C.G. coinciding with the nul position of CM and

$\chi$ is the angular rotation about the vertical axis through the C.G. 
It is assumed that squares and products of $i, \theta, x, \psi$, and $\chi$ are neglected.

The signs are such that if $\mathrm{OY}$ coincides with the length of the pendulum, then (fig. 4)

$$
\begin{aligned}
& x \text { is +along } \mathrm{OX} \\
& \theta \text { is +round } \mathrm{OZ} \text { from } \mathrm{Y} \text { to } \mathrm{X} \\
& \chi \text { is - round } \mathrm{OZ} \text { from } \mathrm{Y} \text { to } \mathrm{X} \\
& \text { and } \psi \text { is +round } \mathrm{OY} \text { from } \mathrm{Z} \text { to } \mathrm{X} \text {. }
\end{aligned}
$$

We have already remarked that except in the immediate vicinity of an earthquake terms arising from $\psi$ and $\chi$ are so small as to be negligible in comparison with those arising from $x$. In that case we may then simply take $x$ as the horizontal

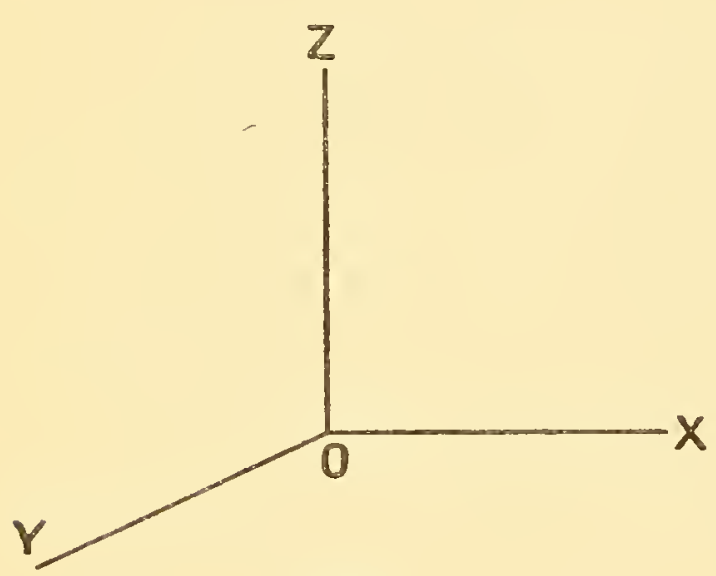

FIG. 4 .

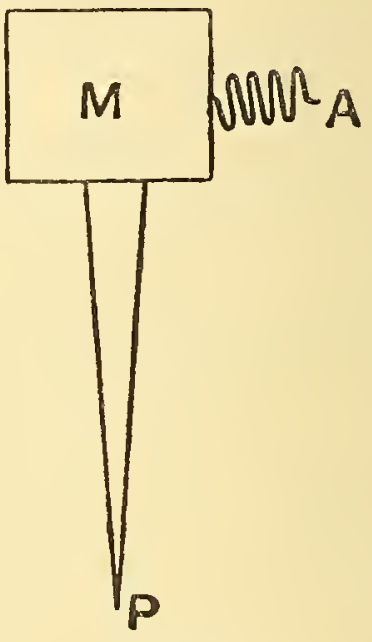

FIG. 5 .

movement of the ground in the vicinity of the seismograph. As showing how small $i$ is in actual instruments we may calculate the value assuming a period of twenty seconds and a length of equivalent simple pendulum ro $\mathrm{cm}$.

$$
\begin{gathered}
\text { We have } \sin i=\frac{4 \pi^{2} l}{T^{2}} \frac{l}{g}=\frac{4 \pi^{2} 10}{400 \times 98 \mathrm{I}} \\
\text { or } i=0^{\circ} 3^{\prime} 5 .
\end{gathered}
$$

The diagram (fig. 5) shows the principle adopted by Wiechert in his seismograph for measuring horizontal movement. It is known as the inverted pendulum.

The mass $M$ is supported by a rigid rod from a fixed point $\mathrm{P}$ about which it can rotate in the plane of the paper. Flat Cardan springs are actually used so as to avoid friction. The 
arrangement would normally be unstable, but it is rendered stable by means of a spring attached to a fixed point $A$ as shown acting horizontally through the C.G. of $M$.

$$
\text { If } \begin{aligned}
M k^{2} & =\text { the moment of inertia about } \mathrm{P} \\
h & =\text { the height of the C.G. of } \mathrm{M} \\
\mu & =\text { the strength of the spring, }
\end{aligned}
$$

then the apparent angular motion $\theta$ of the pendulum is given by

$$
M k^{2} \ddot{\theta}+\left(\mu h^{2}-\mathrm{M} g h\right) \theta=-\mathrm{M} h \ddot{x}
$$

which may be reduced as before to the standard form.

By a suitable choice of $\mu$ sensitiveness can be obtained. In practice the larger pendulums with a mass of $1000 \mathrm{~kg}$. give good results, but with the smaller form in which $M$ is only $80 \mathrm{~kg}$. it is difficult to get a period exceeding eight or nine seconds, as instability occurs when longer periods are attempted.

We may note that this instrument also registers tilting, but not rotation about a vertical axis.

Passing now to the measurement of the vertical motion of the ground, the diagram (fig.

6) represents the principle on which sensitiveness can be obtained. The mass $\mathrm{M}$ carried on a rigid rod PM is capable of moving in a vertical plane about the fixed point P. Flat steel springs

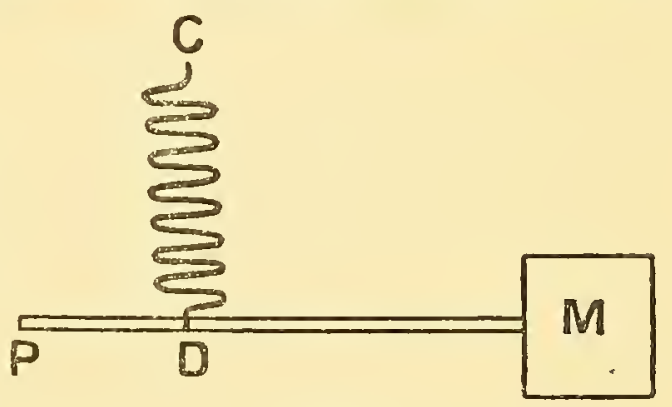

FIG. 6. are actually used to give an axis of rotation through $\mathrm{P}$. The pendulum is supported by a spring $\mathrm{C} \mathrm{D}$ attached to the pendulum at $\mathrm{D}$ and to a fixed point at $\mathrm{C}$.

If $\mathrm{P} \mathrm{D}=d$ and $\mu$ is the strength of the spring, then if $z$ is the vertical earth movement and $\theta$ the apparent angular movement of $\mathrm{P} M$ in the vertical plane we obtain the equation

$$
\mathrm{M} k^{2} \ddot{\theta}+\mu d^{2} \theta=-\mathrm{M} h \ddot{z} \text {. }
$$

By a suitable choice of the quantities $\mu d^{2}$ can be made small and sensitiveness thereby attained.

Introducing a frictional term the equation can be reduced to the standard form. 
We have to note that rotation about an axis perpendicular to the plane of the paper would also be recorded, and that it is most important that the line joining the C.G. and P should be accurately horizontal, otherwise horizontal motion of the ground in the plane of the paper would also contribute to the observed motion.

The best known types are those of Wiechert and Galitzin.

Those familiar with the practical difficulties of mechanical construction will understand that long periods combined with stability are far more difficult to attain for vertical than for horizontal motion. The best result obtained by Galitzin was a period of thirteen seconds. While this is a remarkable practical achievement, it is only about half what can be obtained with horizontal seismographs.

We have found it desirable to retain a frictional term in the equation of motion and we have now to consider this matter more fully.

The Milne Seismograph is the best known throughout the world, and in that instrument no artificial damping is introduced. It is, however, subject to such friction as may exist at the pivot and to the natural damping action of the air. As the pendulum is comparatively light (only about I kg.) we need hardly expect that with reasonable care the effect of the pivot should be serious; and my own experience confirms this. The natural air damping is comparatively small, but conforms as nearly as one can measure on experimental decay curves to the law of proportionality to the velocity. It may be expected to vary somewhat with the temperature and humidity of the air. The Omori Seismograph is also without artificial damping, but as the mass is very great, trouble does arise with the pivot in this case, and the trouble can only be avoided by the use of Cardan springs.

We have observed that when the damping is very slight, the record of an earthquake is largely influenced by instrumental vibration, making it difficult to determine the period and absolute magnitude of the seismic waves, especially when these happen to possess, as they often do, a period nearly that of the pendulum, viz. about eighteen seconds. Thus for 
instance the first phase of an earthquake on a Milne seismogram indicates distinct periodicity, whereas on a Galitzin seismogram the first phase appears extremely irregular. A most interesting confirmation of this came under my notice at Eskdalemuir where, owing to the action of some spiders' threads, the east component of the Milne Seismograph was rendered nearly aperiodic while the north component remained periodic. An earthquake of considerable magnitude occurred, and the profound difference in the appearance of the component records was exceedingly instructive.

For these reasons it has appeared desirable to most investigators to introduce large artificial damping in the seismograph so that the absolute measurement and analysis of seismograms should be rendered easier. Inasmuch as increased damping on a given pendulum would reduce its effective magnification, we must obviously increase the sensitiveness to start with.

The Bosch and Wiechert pendulums are arranged with artificial air damping. This is done by attaching to the pendulum a multiplying arrangement with a piston at the end, so that the piston moves inside a fixed cylinder. The ends of the cylinder are connected by air passages with each other and with the external air, so that the amount of resistance offered to the piston can be varied within certain limits. In this way a frictional term is introduced in the equation of motion and it is possible to attain aperiodicity if so desired.

The results obtained by the use of artificial air damping appear to indicate that it is only as an approximation that the frictional term introduced is proportional to the velocity.

The most important advance in recent years has been made by Galitzin, who successfully introduced electromagnetic damping. A horizontal copper plate attached to the Zöllner pendulum moves in the field produced by a pair of very strong permanent horse-shoe magnets fixed above and below it. The eddy currents induced in the plate when it moves retard the motion, and here there can be no doubt that the retarding force is proportional to the velocity.

Aperiodicity can readily be obtained. The magnets have proved remarkably constant and it is only at intervals of 
several months that they have to be moved a little closer, so
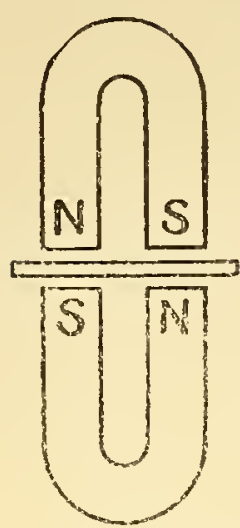

FIG. 7 .

as to increase the field and maintain strict aperiodicity.

We should expect that light pendulums would be used in attempting to get aperiodicity, and it is somewhat curious that the Bosch, Wiechert, and Galitzin pendulums are actually heavier than even the Milne pendulum. We shall consider this in a later chapter.

The values of the angular quantity $\theta$ are small and we have now to consider the manner in which a permanent record of the changes of $\theta$ are obtained on a linear scale of sufficient magnitude. The indicator length $\mathrm{L}$ thus determines the final scale of the record, i.e. the sensitiveness, but I have preferred to keep this separate from the problem of the relation of $\theta$ to the earth movement.

We may imagine a very light but rigid rod of length $\mathrm{L}$ attached to the pendulum, so that the end of the rod gives the linear quantity which is to be registered. This is the method actually used by Milne, an aluminium rod of about I metre length being attached to the pendulum mass.

We cannot, however, practically proceed to great length of a straight rod, so that in some instruments that aim at higher magnification a multiplying arrangement of light levers is introduced. These involve the use of heavier pendulums, and where pivots are used give serious trouble by introducing solid friction and often lead to dislocation of the record owing to loose joints. Galitzin's arrangement of replacing pivots by fine wire and spring control gets rid of this objection.

The indicating end may be made to write by means of a style on smoked paper or by a pen with ink on smooth white paper. Of the sharpness of the lines so obtained there is no question and its cheapness is a great recommendation. It is used in the Wiechert and Omori Seismographs. Unfortunately solid friction is introduced by mechanical registration, the fundamental equation of motion has to be modified, and recent investigation has made it doubtful whether the matter can be dealt with in a satisfactory way. 
But to Milne we owe the application of photography to the problem of recording, without the introduction of any friction or backlash of multiplying levers. The precise method used by Milne will be described in the next chapter.

The newer method of photographic registration used in the Bosch and Galitzin instruments depends on the principle that a pencil of light from a strong source of illumination may be reflected from a mirror attached to the pendulum and concentrated at a point on the surface of the sensitive paper. An indicating point is thus obtained without introducing the slightest friction, and so the simple mathematical form of the fundamental equation is preserved.

The most recent method of multiplying the motion of a pendulum before applying photographic registration we owe to Galitzin. It occurred to him that if a coil of fine wire was attached to the pendulum, so as to cut across the lines of a strong permanent magnetic field when the pendulum moved, electrical currents would be set up in the coil, strictly proportional to the angular velocity of the pendulum. These currents could be carried by wires to a recording galvanometer, so that the movement of the needle would register photographically on a large scale the motion of the pendulum and hence of the earth movement.

The motion of the pendulum being given as before by

$$
\ddot{\theta}+2 \epsilon \dot{\theta}+n^{2} \theta=-\ddot{x} / l
$$

then the equation for the angular motion $\phi$ of the galvanometer needle can be written in the form

$$
\ddot{\phi}+2 \epsilon_{1} \dot{\phi}+n_{1}{ }^{2} \phi=-k \dot{\theta}
$$

where $e_{1}, n_{1}$, and $k$ are certain instrumental constants. There is thus linear relation between $\phi$ and $x$.

This is the principle of Galitzin's electromagnetic registration method, where in practice both pendulum and galvanometer are made to have the same period and be aperiodic within very narrow limits. 


\section{CHAPTER III.}

THE CHIEF TYPES OF SEISMOGRAPHS IN ACTUAL USE.

The Milne Seismograph is made by Mr. R. W. Munro, London, and to him and to Dr. Milne I am indebted for permission to use the photograph shown on Plate I. The supporting frame of the pendulum consists of a vertical iron pillar cast in one piece with a triangular bed-plate supported on three levelling screws, which rest by hole, slot, and plane on three glass studs imbedded in the pier. The pendulum boom is a light rod of aluminium nearly I metre long, and at the inner end it is fitted with an agate cup which presses against a steel pivot point screwed into the pillar. The boom is supported at a point a little beyond the stationary mass by means of a fine steel wire ending in unspun silk which passes to a pin at the top of the pillar. The mass (about I kg.) itself is not rigidly attached to the boom, but is balanced on a steel pivot. The object of this appears to be to reduce the effective moment of inertia of the pendulum. The adjustments provide for bringing the boom into the horizontal position along a prescribed line, and so as to have the desired period of say eighteen seconds. One of the levelling screws, having a pitch $0.5 \mathrm{~mm}$., carries an arm moving over a graduated arc, and provides the means of giving a known tilt to the instrument, so that its static sensitiveness may be determined. The boom is prevented from sagging at its outer end by a silk cord as shown.

The registration is carried out as follows: The boom carries at its end a small transverse plate of aluminium with a narrow slit parallel to the boom. This moves over a fixed slit at right angles to it in the top of the registration casing. This arrangement is illuminated from above so that a small dot of light corresponding to the intersection of the slits is cast 


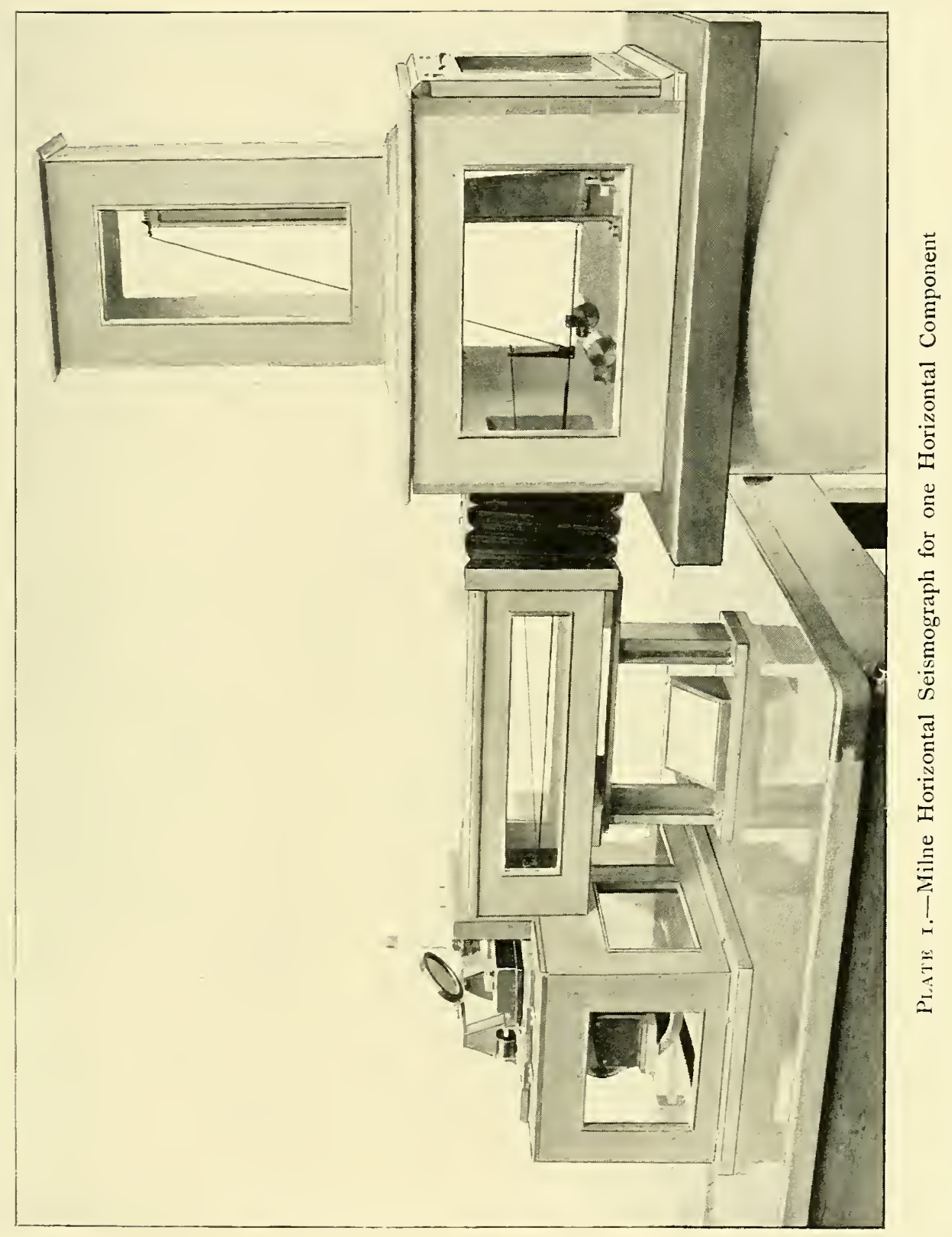






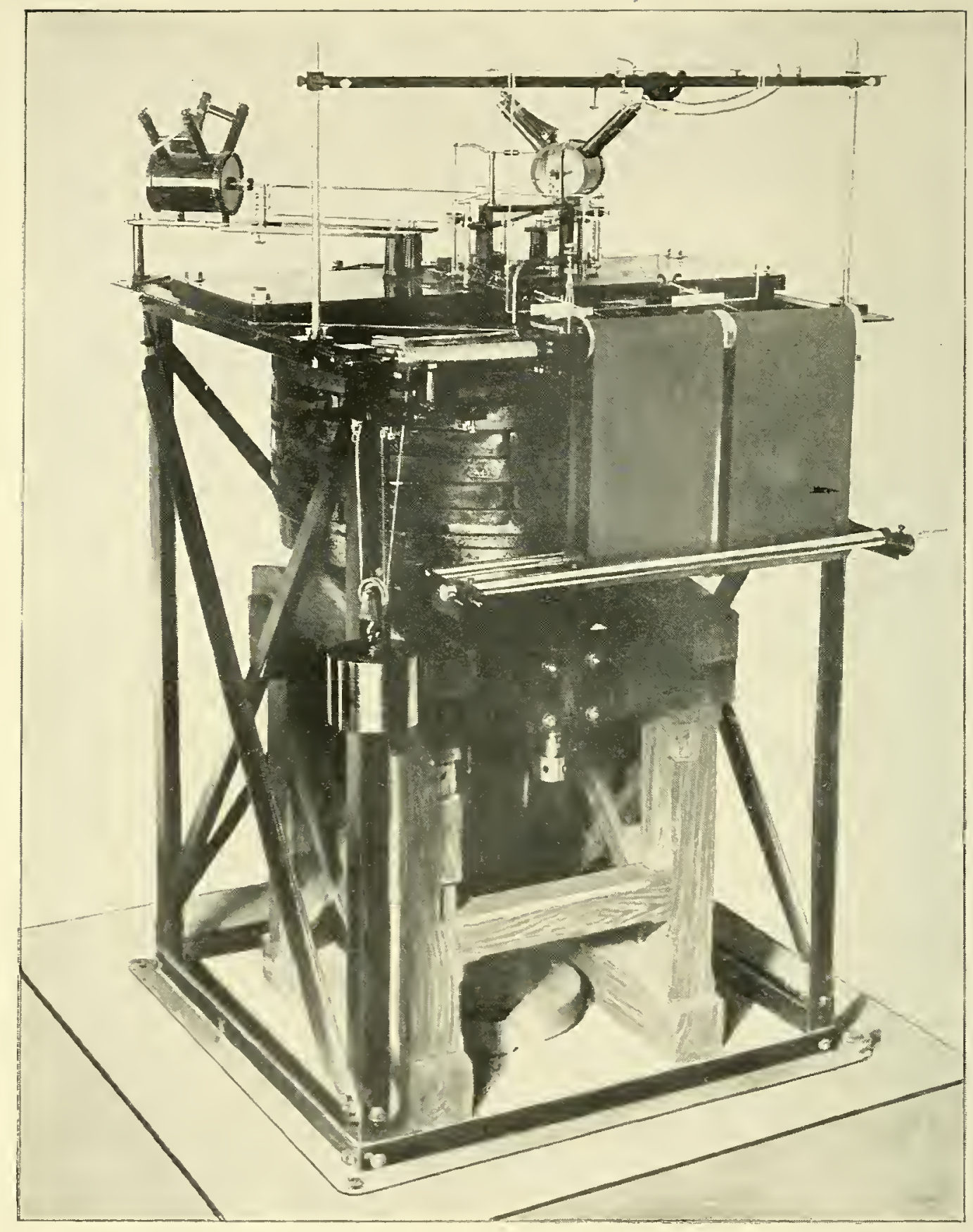

Plate 2.-Wiechert Astatic Inverted Pendulum Seismograph for two Horizontal Components 
on the surface of the bromide paper wound on the surface of the recording cylinder. The cylinder driven by a spring clockwork rotates in about four hours, the paper speed being about $250 \mathrm{~mm}$. per hour or nearly $4 \mathrm{~mm}$. per minute. By means of a deep helix cut in the spindle the cylinder is made to move sideways as it revolves by about $6 \mathrm{~mm}$. per revolution, so that the paper is available to run for one day or two days as the case may be. Every hour the light is cut off by an electric shutter operated by a good pendulum clock, so that accurate time marks are thus put directly on the record and eliminate any irregularity in the driving spring clock, which cannot be depended on to give sufficiently accurate time.

In the twin boom instrument the two pendulums are carried at right angles by the vertical pillar, but the booms are brought out parallel to each other so that the two horizontal components are recorded on one sheet.

The instrument is subject only to such natural damping as may occur, and this is very small.

The Omori Seismograph resembles the Milne instrument, but is much larger, the stationary mass being about Ioo $\mathrm{kg}$. A multiplying lever and registration on smoked paper is also used.

The Bosch Seismograph is also similar to the Milne, but of a somewhat heavier build. It is fitted with artificial air damping and registers photographically by means of a mirror attached to the pendulum.

The Wiechert Iooo $\mathrm{kg}$. astatic pendulum is made by Herr Bartels, Göttingen. To him and to Prof. Wiechert I am indebted for permission to use the photograph, Plate 2. We must refer also to the diagrammatic sketch in Chapter II.

The stationary mass is built up of iron plates and supported by a strong iron pillar from the pier. The support consists of a double set of Cardan springs so as to avoid friction and allow the pillar and mass to rotate about two horizontal axes at right angles to each other. A rigid framework carries the registering apparatus, supports for the damping boxes, etc. Stops are also provided to prevent any large motion of the mass, which would be fatal to the instrument. 
Two light arms engage with points on the top of the mass so as to give the components of motion in the horizontal direction. These two arms are connected up to the writing points by means of a system of similar multiplying levers. We must refer to the "Phys. Zeit.," p. 82I, I903, for full details as to these. It must suffice here to say that connexions are provided by steel points working in agate cups while axes of rotation of levers are provided by small Cardan springs which also provide the small controlling force required to make the pendulum stable. Air-damping boxes are also attached so as to provide any required degree of damping. The registration is made on smoked paper in a manner clearly indicated in the photograph, the writing point being a glass style with a small ball point.

The whole arrangement is of great delicacy and requires very careful handling so as to avoid damage to any part.

The speed of registration is about $10 \mathrm{~mm}$. per minute, and automatic time marks are put on the record every minute from a good pendulum clock which operates an electrical arrangement for lightly raising the writing points and then lowering them, so that a small break occurs on the trace. The dimensions of the enclosing case are $186 \mathrm{~cm}$. high, I 38 $\mathrm{cm}$. broad, and $176 \mathrm{~cm}$. long. It is unfortunately true that in this seismograph the two components are not independent.

The Galitzin Seismographs are made by Mr. Massing, mechanic in the Imperial Academy of Sciences, St. Petersburg. I am indebted to His Excellency Prince Galitzin for permission to use the photographs, Plates 3 and 4 .

Plate 3 shows the horizontal component seismograph with galvanometer and recording drum. The general dimensions of the seismograph may be judged from the fact that the casing is about I $\mathrm{m}$. high and rather less than I $\mathrm{m}$. diameter.

The base is a substantial iron casting supported on three strong levelling screws. Bolted to this is a rigid iron framework which carries the horse-shoe magnets and clamps for the supporting wires.

The pendulum which is of Zöllner type consists of a strong brass rod to which is rigidly keyed the stationary mass of $7 \mathrm{~kg}$. 


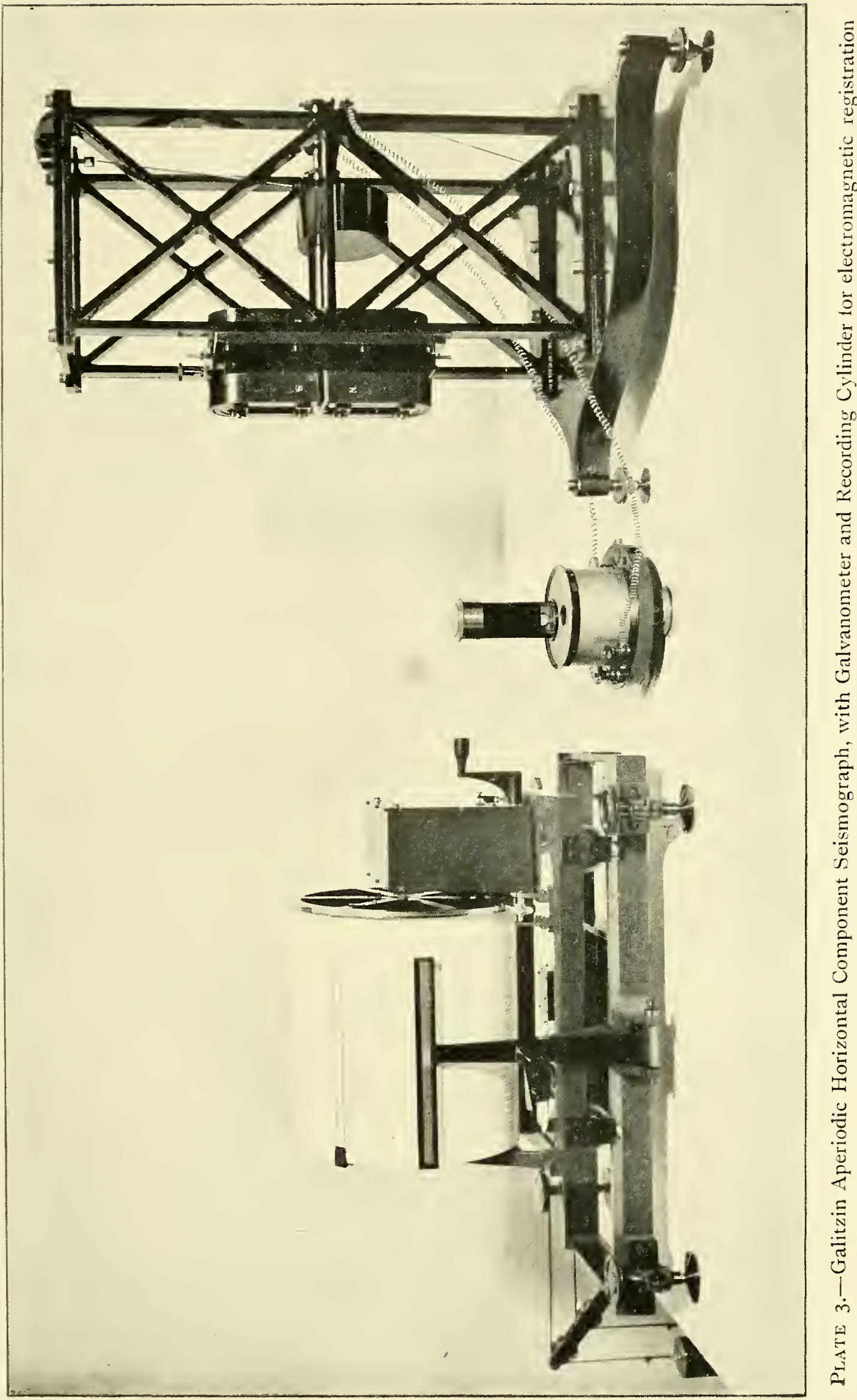



The supporting wires are of steel and platinum-iridium and pass one from the top of the frame to a point just behind the mass, and the other from the inner end of the rod to the foot of the frame.

At the outer end of the pendulum rod are seen the copper plate and damping magnets, while just behind these are seen the similar pair of magnets which excite the currents in the flat coils attached to the pendulum. These coils are connected to the stout leads of the galvanometer, the connexion being made by fine bronze strips so as not to interfere with the free movement of the pendulum. Both pairs of magnets are provided with screw adjustments so that the damping and magnification may be controlled to the desired extent. Small mirrors are attached to the pendulum and frame, and these with a small electric hammer for giving the pendulum a slight blow are required in the process of standardizing.

The galvanometer is of the Deprez-D'arsonval moving coil type.

The registration of the movements of the galvanometer mirror is made photographically. The galvanometer is set at a convenient distance, say $\mathrm{I} \mathrm{m}$., from the recording cylinder, and the mirror is illuminated by means of collimator and slit, so that the reflected beam falls normally on the cylinder, while the image is focussed for that distance. The image is concentrated to a small luminous point by the intervention of a cylindrical lens. The cylinder has a circumference of nearly I $\mathrm{m}$. and revolves in about half an hour, so that the actual paper speed is $30 \mathrm{~mm}$. per minute. The cylinder also moves sideways about I $\mathrm{cm}$. per revolution so that the record runs for twelve hours. In practice we may arrange for both horizontal components, from duplicate pendulums set at right angles, to be recorded side by side on the same sheet. This is an economy of expense and possesses certain distinct advantages. But against this must be set the fact that when a large earthquake occurs the confusion of the record may become very troublesome. Thus at Pulkowa the practice is to record each component on a separate sheet, while two scales of registration are used. 
Plate 4 shows the Galitzin vertical component seismograph for electromagnetic registration. The general dimensions of the casing are rather over $\mathrm{I} \mathrm{m}$. in length and less than $\mathrm{I} \mathrm{m}$. in breadth and height. The pendulum rod is now replaced by a framing to avoid bending. The axis of rotation is very neatly arranged to avoid friction by using crossed Cardan springs screwed to the fixed framing and to the pendulum framing.

The strong supporting spiral spring is fitted with a screw to get rough adjustment, while final adjustment is made by a small gravity weight shown to the left of the stationary mass. Another adjustable mass shown above the axis of rotation is provided to get the centre of gravity of the pendulum in the same horizontal line with the axis of support.

The arrangement of horse-shoe magnets is similar to that in the horizontal seismographs, but they have to be twice as large, as the attainable period is about half that of the horizontal instrument. Mirrors and electric hammer are provided for standardization and the registration is made exactly as in the case of the horizontal components.

Although these seismographs are far more sensitive than either the Milne or Wiechert Seismograph, they are by no means difficult to handle. The chief danger to avoid is making any adjustment of the pendulum while the galvanometer is in circuit with it.

Further details will be found in Prince Galitzin's “ Lectures on Seismometry," published (in Russian) by the Imperial Academy of Sciences, St. Petersburg. These lectures embody the chief results of separate investigations published (in German) in the Comptes Rendus, Imperial Academy of Sciences, St. Petersburg, and the Permanent Seismological Commission, St. Petersburg. 


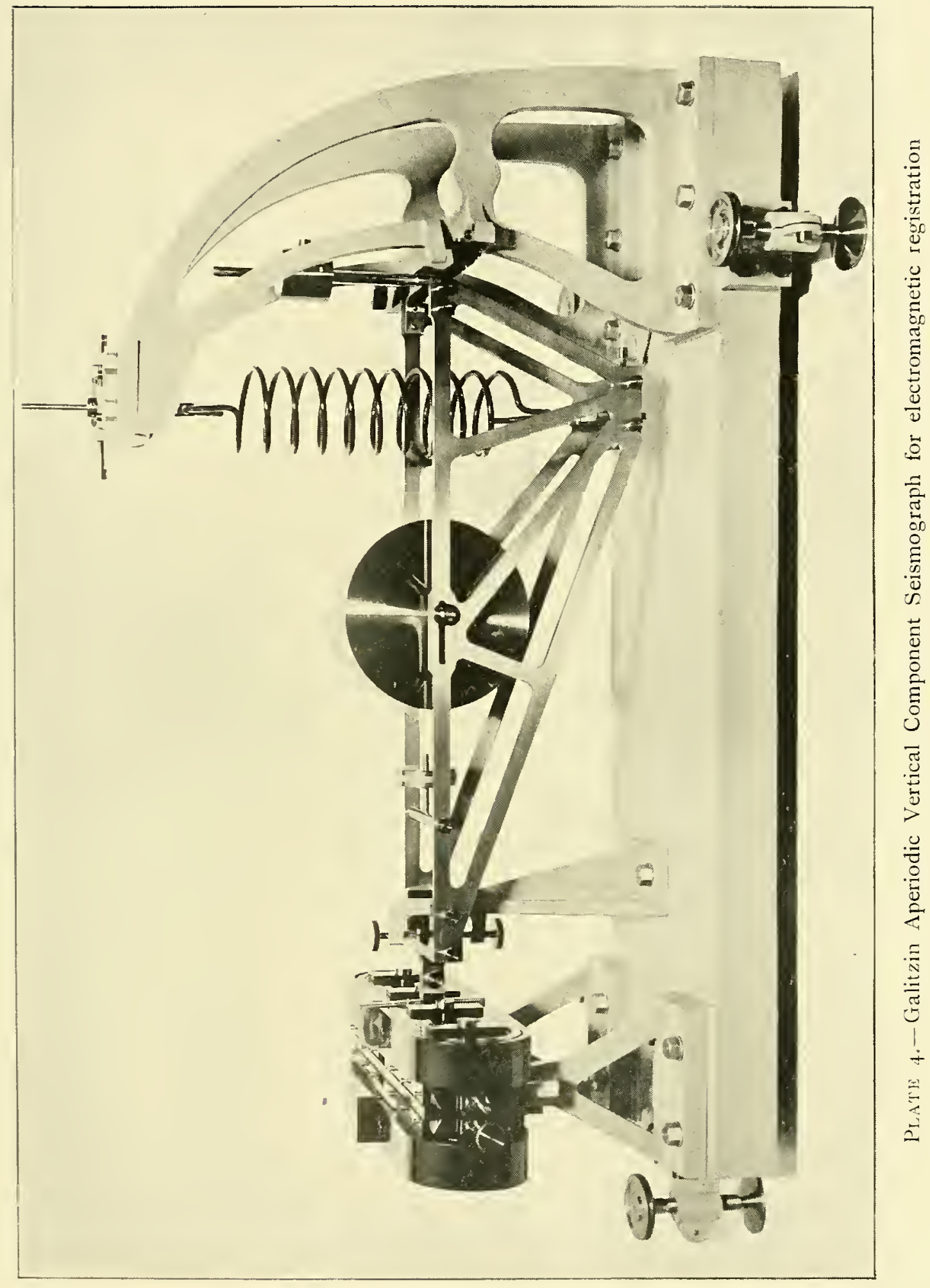





\section{CHAPTER IV.}

STANDARDIZATION OF SEISMOGRAPHS INCLUDING THEORY OF MECHANICAL AND ELECTROMAGNETIC REGISTRATION.

IN order that the study of seismograms should contribute in real measure to a knowledge of geophysics, it is essential that the results obtained should be expressed in absolute measure. When we remember that we have to compare records obtained at different stations with instruments, it may be, of the same or even of different types, the necessity for standardization becomes evident. Not only so, but since any individual instrument undergoes secular change and requires readjustment from time to time, it must be possible to determine the constants of the instrument in situ at suitable intervals.

In the first instance it is, however, important that each instrument should be tested in order to ascertain whether it conforms to the fundamental equation supposed to represent its motion. This can only be done on a properly equipped experimental table by some central recognized authority, which would then issue with the instrument a certificate giving any data of importance.

We shall consider how the constants are to be obtained at the station for the three well-known types, Milne, Wiechert, and Galitzin.

The latest form of Milne's twin-boom Seismograph readily lends itself to the determination of the quantities $\epsilon$ and $n$ on the photographic sheet itself, a point of great practical convenience. If the end of the boom is given a suitable initial displacement and then left to itself, excellent decay curves are obtained. The diagram (fig. 8) is an exact reproduction of an actual curve obtained in this way. 
The paper speed is $4 \mathrm{~mm}$. per minute, and for a period of I $8^{s}$ this implies $24 \mathrm{~mm}$. on the paper for twenty vibrations.

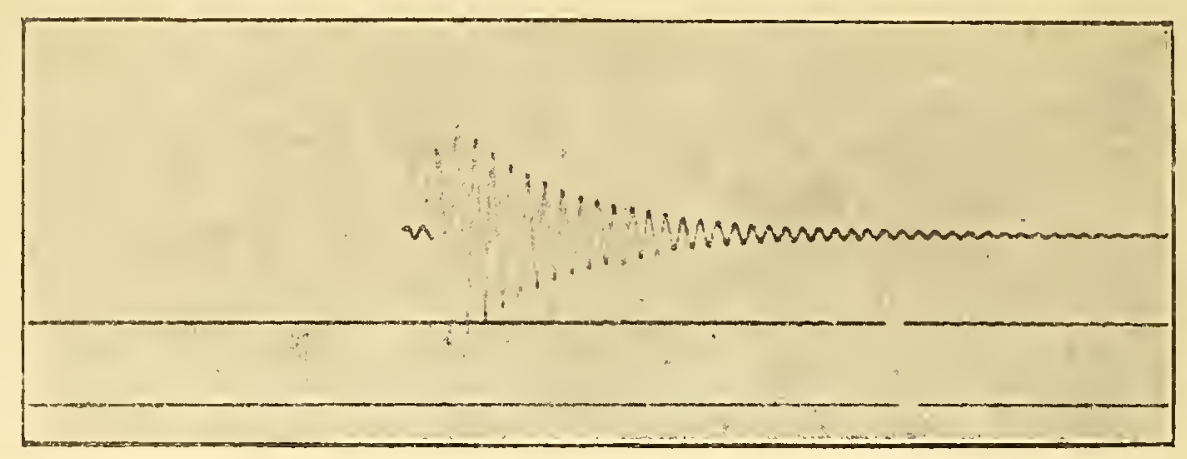

Fig. 8.

There is thus no difficulty in measuring the apparent period $\mathrm{T}^{\prime}$ to $\mathrm{O}^{s \cdot} \mathbf{I}$. We may also determine with considerable accuracy the ratio of successive amplitudes by measuring the ratio for say ten vibrations. We may then compute $n$ and $\epsilon$ as follows :-

Since

$$
\mathrm{T}^{\prime}=2 \pi /\left(n^{2}-\epsilon^{2}\right)^{\frac{1}{2}}
$$

and the ratio of successive amplitudes

$$
v=e^{\epsilon \mathrm{T}^{\prime} / 2}
$$

we find that

$$
\epsilon=\frac{2}{\log _{10} e \mathrm{~T}^{\prime}}=4^{\circ} 605 \lambda / \mathrm{T}^{\prime}
$$

where

$$
\lambda=\log _{10} v
$$

and

$$
n=\frac{2 \pi}{\mathrm{T}}=\frac{2 \pi}{\mathrm{T}^{\prime}}\left\{\mathrm{I}+\lambda^{2} /(\pi \log e)^{2}\right\}^{\frac{1}{2}}=\frac{2 \pi}{\mathrm{T}^{\prime}}\left\{\mathrm{I}+0.5372 \lambda^{2}\right\}^{2} .
$$

From the curve (fig. 8) I found fifteen vibrations in $19 \mathrm{~mm}$. and the amplitude fell from $5 \mathrm{~mm}$. to $I \mathrm{~mm}$. in ten vibrations, so that we get $\mathrm{T}^{\prime}=\mathrm{I} 9^{\mathrm{s}} \cdot \mathrm{O}$ and $v=\mathrm{I} \cdot 084$ and so $\mathrm{T}=\mathrm{I} 9^{\mathrm{s}} \cdot \mathrm{O}$, $n=0.33 \mathrm{I}$, and $\epsilon=0.0085$.

We have pointed out that Milne has always provided a screw by which a known static tilt can be given to the instru- 
ment and the observed deflection on the paper noted, and with his published data of amplitudes in millimetres he gives the angular tilt required to produce $\mathrm{I} \mathrm{mm}$. deflection.

Now the fundamental equation

$$
\ddot{\theta}+2 \epsilon \dot{\theta}+n^{2} \theta=-\ddot{x} / l+g \psi / l
$$

shows that for a steady tilt $\psi_{1}$ we get

$$
\theta_{1}=\frac{g \psi_{1}}{n^{2} l}
$$

and since the deflection on the paper say $y_{1}$ is $\mathrm{L} \theta_{1}$ where $\mathrm{L}$ is the length of the boom we may calculate $l$ by the formula

$$
l=\frac{\operatorname{Lg} \psi_{1}}{n^{2} y_{1}}
$$

As an actual example we have

$$
\mathrm{L}=100 \quad n=\frac{2 \pi}{\mathrm{I} 8}=0.349
$$

and the experiment gave a deflection of I mm. for $\mathrm{O}^{\prime \prime} \cdot 43$ tilt so that $l=\mathrm{I} 6 \cdot 8$ and $\mathrm{L} / l=6$.

$L$ and $l$ are of course constants that may be determined once for all. Thus while we must admit that in a complicated record it would be practically impossible on account of the "free" terms to assign the true magnitude of the horizontal earth movement in absolute measure, there are certain cases (notably sharp impulses) in which the earth movement can be determined from the record. This point has not always been recognized with regard to the Milne Seismograph.

In the Wiechert Seismograph artificial air damping is introduced. We shall first suppose that the friction introduced enables us to write the equation in the form

$$
\begin{gathered}
\mathrm{M} k^{2} \ddot{\theta}+\lambda \dot{\theta}+\left(\mu h^{2}-\mathrm{Mg} h\right) \theta=-\mathrm{M} h \ddot{x} \\
\text { or } \ddot{\theta}+2 \epsilon \dot{\theta}+n^{2} \theta=-\ddot{x} / l .
\end{gathered}
$$

When the expression $\mathrm{I} / \mathrm{U}$ which determines the magnification is plotted for different values of $u$ and of the damping ratio $v$, it appears that the magnification remains more nearly constant from $u=0$ to $u=\mathrm{I}$ when $v$ is about 5 than for other values of $v$, and this ratio is aimed at in practice. The corresponding value of $\epsilon / n$ is about 0.45 . This comparatively large damping ratio makes it difficult in practice to get a sufficient 
number of vibrations on the paper when an artificial disturbance is given, to determine the apparent period $\mathrm{T}^{\prime}$ exactly. Hence the artificial damping is cut out so that $\epsilon$ is reduced considerably to $\epsilon^{\prime}$, and $\epsilon^{\prime}$ and $n$ are then determined exactly as we should do in the case of the Milne instrument. As the paper speed is one minute $=10 \mathrm{~mm}$. it is generally possible to determine $\mathrm{T}^{\prime}$ to $\mathrm{O}^{6} \cdot \mathrm{I}$.

The damping is again introduced, and after getting $v$ nearly 5 by trial, its exact value is obtained and $n$ being now known $\epsilon$ is determined. The period $\mathrm{T}=2 \pi / n$ aimed at is from $10^{s}$ to $12^{s}$ in the $1000 \mathrm{~kg}$. instruments.

Since the instrument also measures tilt we might theoretically now get $l$, by giving a known static tilt. But it is not easy practically to give a known tilt to the $80 \mathrm{~kg}$. pendulum, and in the case of $17,000 \mathrm{~kg}$. pendulum it is out of the question. Hence resort is had to another way (cf. "Phys. Zeit.," I 903, 1.c.). If a small known mass $m$ say Io gm. is placed on the large mass $M$ at a distance $p$ of say $I 0 \mathrm{~cm}$. horizontally from the centre of gravity, a small couple is produced which gives an angular deflexion that may be conveniently measured on the paper.

We have

and

$$
\left(\mu h^{2}-\mathrm{M} g h\right) \theta_{1}=m g p
$$

$$
\mathrm{L} \theta_{1}=y_{1}
$$

where $y_{1}$ is the deflexion on the paper and $L$ is known from the linear dimensions of the multiplying levers. Hence we obtain $\left(\mu h^{2}-\mathrm{Mgh}\right)$ and hence knowing $n$ we get $l$. As $l$ is proportional to $\mathrm{L}$ we really do not require to know $\mathrm{L}$ in getting the magnification. The value of $\mathrm{L} / l$ is readily made several hundred units.

Wiechert, however, recognized that the mechanical registration introduced frictional forces that are not properly allowed for by a term proportional to $\dot{\theta}$. He assumes (Theorie Auto. Seis.) that the solid friction was such that the equation took the form

$$
\ddot{y}+2 \epsilon \dot{y}+n^{2}(y \pm r)=0
$$

where the sign of $r$ is such as to oppose the motion, reversing 
whenever $y$ reverses. If now $y_{1}, y_{2}, y_{3}$ represent successive amplitudes while $v$ is the ratio of successive amplitudes of the periodic term, it is easy to see that

$$
v=\frac{y_{1}-r}{y_{2}+r}=\frac{y_{2}-r}{y_{3}+r}=\text { etc. }
$$

and hence

$$
v=\frac{y_{1}-y_{2}}{y_{2}-y_{3}}=\frac{y_{1}+y_{2}-2 r}{y_{2}+y_{3}+2 r}
$$

while

$$
r=\left(y_{2}^{2}-y_{1} y_{3}\right) /\left(y_{1}-y_{3}\right)
$$

which suffice to determine $r$ and $\epsilon / n$. In practice it is best to obtain $r$ when the damping is cut out, so that $v$ being nearly I we get approximately $r=\frac{1}{4}\left(\gamma_{1}-y_{3}\right)$.

The writing point may remain at rest anywhere within a range $2 r$, and discontinuities of this magnitude may occur in the trace.

It is also clear that the motion can never start unless the impressed acceleration exceeds a certain amount, and this explains the fact that so many more earthquakes are recorded on instruments that use photographic registration even with smaller magnification.

But a more serious matter arises. Experiments of my own on an $80 \mathrm{~kg}$. Wiechert showed clearly that $r$ was not a constant, but depended on the state of the smoked surface and the amplitude of the movement. This has been more recently established by Galitzin (Vorles. ii. Seis.) whose elaborate experiments show that a more complex and non-linear equation corresponds better with the facts. This, however, rather suggests that if cases arise where the solid friction is so great as to seriously vitiate the records, we should do well frankly to abandon mechanical registration. With reasonable care the magnitude of $r$ does not exceed a few tenth millimetres in the Wiechert instruments.

The motion of the Galitzin horizontal pendulum is given by the usual equation

$$
\ddot{\theta}+2 \epsilon \dot{\theta}+n^{2} \theta=-\ddot{x} / l \text {. }
$$

In practice $T=$ nearly $24^{s}$ or $n=0.26$ I 8 , and when in use we make $\epsilon$ as nearly $=n$ as possible. The length $l$ is a definite 
constant for each instrument and is about $\mathrm{I} 20 \mathrm{~mm}$. It may be determined by observing the static sensitiveness when $n$ has some known value.

Another method used by Galitzin depends on the principle that $n^{2}=g i \mid l$, so that by changing $i$ by known amounts and determining the corresponding values of $n$ we get data for calculating $l$.

Thus $l=g\left(i_{1}-i_{2}\right) /\left(n_{1}^{2}-n_{2}^{2}\right)$.

The artificial damping is cut out by removing the magnets, and observation of the apparent period and damping ratio (now nearly unity) is made, so that the values of $n$ can be computed. The changes of $i$ are determined by observation of a mirror attached to the frame, by means of telescope and scale.

The values of $\epsilon$ and $n$ do not remain quite constant, so that it becomes important to determine them at any time in situ.

The differences of $\epsilon$ and $n$ from their theoretical values are small, and are determined by observations on the recording galvanometer, the theory of which we have yet to consider.

The galvanometer is of the moving coil type in which the suspended system is controlled by the torsion of a fine wire. When a current I exists in the coil a couple strictly proportional to $I$ arises, due to the strong magnetic field in which the coil turns. If we neglect the self-induction of the circuit the current $\mathrm{I}=\mathrm{E} / \mathrm{R}$ where $\mathrm{E}$ is the electromotive force in the circuit and $\mathrm{R}$ the resistance. When connected to the coil in the pendulum $\mathrm{E}$ consists of two parts: (I) due to the pendulum motion and proportional to $\dot{\theta}$, and (2) due to the motion of the galvanometer coil and proportional to $\dot{\phi}$.

Thus we see that the equation of motion of the galvanometer coil is given by

where $\epsilon_{1}=c_{0}+c / \mathrm{R}$.

$$
\ddot{\phi}+2 \epsilon_{1} \dot{\phi}+n_{1}^{2} \phi=-k \dot{\theta}
$$

When on open circuit $(R=\infty)$ the free motion of the coil is given by

$$
\ddot{\phi}+2 c_{0} \dot{\phi}+n_{1}^{2} \phi=0
$$

so that $c_{0}$ arises from a small damping action to which the system is naturally subject. 
The quantities $c_{0}$ and $n_{1}$ are determined for the galvanometer in the usual way. The period is about $24^{5}$ while $c_{0}$ turns out to be a very small quantity of order 0.0005 .

When the circuit is closed we see that $\epsilon_{1}$ increases as $\mathrm{R}$ diminishes and thus the condition of true aperiodicity $\epsilon_{1}=n_{1}$ can be secured by suitable choice of $R$. The value is determined by experiment thus: Different values of $R$ are introduced and the corresponding values of $\epsilon_{1}$ determined. In this way $c$ is found and is a quantity of order 6 units. We may then calculate the value of $\mathrm{R}$ required to make $\epsilon_{1}=n_{1}$, and find it to be about 25 ohms. The galvanometer resistance is about 4 ohms and thus the remainder of the circuit must be made up to the required value. When this is done the galvanometer is assumed to be aperiodic $\left(\epsilon_{1}=n_{1}\right)$ and to remain so as the quantities involved are not subject to changes that have any appreciable effect.

Having granted the desirability of great damping, the passage to the limit of aperiodicity seems obvious as it simplifies the relation between the quantities. Thus the ideal is to have the pendulum and galvanometer truly aperiodic and to have the same primary period in the absence of damping, i.e. $\epsilon=\epsilon_{1}=n=n_{1}$. Assuming then that $\epsilon_{1}=n_{1}$ we proceed by trial to make $\epsilon=n=n_{1}$, or $\mu^{2}=0$, and it is easy to get quite near it. The adjustment would in fact not be considered good if $\mu^{2}$ rose to $O \cdot I$ or if the primary periods differed by more than a few tenths of a second. The pendulum does, however, undergo small secular changes, and we have now to explain how Galitzin determines how far $n$ differs from $n_{1}$, and $\mu^{2}$ from $o$, and also the value of $k$ the transference factor of order about forty units.

To simplify matters, suppose the ideal condition secured and that a small impulse is given to the pendulum. Then

$$
\theta=n_{1} e \theta_{m} e^{-n_{1} t} t
$$

so that $\theta_{m}$ is the maximum value of $\theta$.

The corresponding motion of the galvanometer needle is given by

so that $\phi=\dot{\phi}=0$ when $t=0$.

$$
\phi=-k n_{1} e \theta_{m} e^{-n_{1} t}\left(\frac{t^{2}}{2}-\frac{n_{1} t^{3}}{2 \cdot 3}\right)
$$


We note that $\phi$ is again o when $t=t_{0}=3 / n_{1}$.

Again $\phi$ is a maximum when

$$
\begin{aligned}
& n_{1}{ }^{2} t^{2}-6 n_{1} t+6=0 \\
& \text { or } n_{1} t=3 \pm \sqrt{3}
\end{aligned}
$$

Thus the first maximum is

$$
\phi_{1}=\frac{k \theta_{m}}{n_{1}}(2 \sqrt{3}-3) e^{-2+\sqrt{3}} \text { when } t=(3-\sqrt{3}) / n_{1}
$$

$\phi$ then passes through $\circ$ when $t=3 / n_{1}$ and attains a maximum on the other side

$$
\phi_{2}=\frac{k \theta_{m}}{n_{1}}(2 \sqrt{3}+3) e^{-2-\sqrt{3}} \text { when } t=(3+\sqrt{3}) / n_{1}
$$

and then $\phi$ gradually diminishes to $O$ when $t=\infty$. Hence

$$
k=n_{1} \frac{\phi_{1}}{\theta_{m}} \frac{e^{2}-\sqrt{3}}{(2 \sqrt{3}-3)}=n_{1} \frac{\phi_{2}}{\theta_{m}} \frac{e^{2+\sqrt{3}}}{(2 \sqrt{3}+3)} .
$$

Now if $\epsilon$ and $n$ differ a little from $n_{1}$, the motion of $\phi$ will differ from the above, but without altering the essential feature that $\phi$ attains maximum throws on opposite sides. The complete equations can be written down and observation of $\theta_{n n}, \phi_{1}, \phi_{2}$, and $t_{0}$ then provide material for calculating $k, \mu^{2}$, and $\left(n-n_{1}\right)$. The necessary formulæ and numerical tables have been obtained by Galitzin (1.c. ante). It must suffice here to point out that for all practical purposes the following are quite accurate enough, viz. :-

$$
\begin{aligned}
\frac{n_{1}-n}{n_{1}} & =\xi=\frac{2}{3}\left(n_{1} t_{0}-3\right) \\
\mu^{2} & =2.94\left(2.294 \frac{\phi_{2}}{\phi_{1}}-\mathrm{I}\right) \\
k & =2.8 \mathrm{I} 7 n_{1} \phi_{1} / \theta_{m}=6.46 n_{1}\left(\mathrm{I}-0.34 \mu^{2}\right) \phi_{2} / \theta_{m}
\end{aligned}
$$

when $\xi$ and $\mu^{2}$ do not exceed $0 \cdot 1$. It is of interest that the effect of $\mu^{2}$ is much greater in changing $\phi_{2} / \theta_{m}$ than it is in changing $\phi_{1} / \theta_{m}$.

Having determined $n_{1}$, with the galvanometer on open circuit, the procedure is to give the pendulum a small impulse with a small electrically controlled hammer, and then to observe by aid of telescopes and scales the quantities $\theta_{n}, \phi_{1}, \phi_{2}$, while $t_{0}$ is determined by a chronograph. Two observers are required 
and considerable skill is necessary. About ten experiments are made so as to improve the accuracy.

The standardization of the vertical component seismograph proceeds in precisely the same manner as regards $\xi, \mu^{2}$, and $k$. In this case the primary period is about $\mathrm{I} 3^{s}$ while $k$ is of order 240 units. The quantity $l$ of order $400 \mathrm{~mm}$. is determined before the instrument is set up by removing the controlling spring, turning the instrument so that the pendulum may hang vertically, and observing the period of vibration. When in use a small correction may be made on account of the position of the small adjustable gravity weight.

In the Galitzin Seismographs the indicating length is $2 \mathrm{~A}$ where $A$ is the distance from the galvanometer to the recording drum, usually chosen to be about $100 \mathrm{~cm}$. Thus if the theoretical adjustment has been secured the magnification for periodic waves is

$$
\frac{\mathrm{A} k \mathrm{~T}_{1}}{\pi l} \frac{u}{\left(\mathrm{I}+u^{2}\right)^{2}} \text { where } u=n / p=\mathrm{T} / \mathrm{T}_{1}
$$

The expression is a maximum for $u=1 / 3^{\frac{1}{2}}=0.577$ and the value of $u /\left(I+u^{2}\right)^{2}$ is then 0.325 . Thus the magnification is nil for very rapid vibrations, rises to a maximum, and then falls again to $O$ for very slow vibrations. As an example, if $\mathrm{T}_{1}=24 \mathrm{sec}$, $\mathrm{A}=\mathrm{I} 00 \mathrm{~cm} ., l=\mathrm{I} 2 \mathrm{~cm} ., k=40$ the maximum magnification would be 828 when $\mathrm{T}=\mathrm{I} 4 \mathrm{sec}$. 


\section{CHAPTER V.}

INSTALLATION OF SEISMOGRAPHS AND CONSIDERATION OF DEVELOPMENT OF INSTRUMENTS.

THE site of a seismological station is probably determined in most cases by considerations of policy and finance which do not concern us here. But we may consider some conditions that appear desirable from a scientific point of view.

Seismographs are sensitive instruments and thus liable to be disturbed by artificial causes such as street traffic, so that the instruments ought to be installed at some distance from a town or railway line. But such local effects do not penetrate to a great distance, so that it is only a question of being a kilometre or so distant from such a source of disturbance. We remember that most of the European stations from which such important results have been obtained are at no great distance from busy centres of industry. The dominant features of a seismogram of a large tectonic earthquake are not determined by local conditions of the ground, but the smaller details of the seismograms may be modified greatly by the geological formation of the rocks in the vicinity of the station. Thus a site where the formation is known to be of fairly uniform character for a considerable area would be preferable to one where the rocks vary rapidly. A level plain also recommends itself, while a sharp ridge or sudden depression are to be avoided. If we remember, however, that the most frequent wave lengths experienced are from 30 to $70 \mathrm{~km}$. long, the presence of an isolated obstacle in the form of a hill is probably not a very serious matter.

There are few recording instruments of any kind that are not prejudicially affected by change of temperature, and thus uniformity of temperature in the room containing the seismographs is a highly desirable condition even from a general 
point of view, and becomes of vital importance if the movements of the pendulum zero are to be examined for diurnal tilting of the ground due to earth tides. An underground chamber may be used to conduce to such uniformity of temperature, and it has been found at Pulkowa that the disturbing effects of local wind are considerably reduced in an underground room.

The pendulums have to be carried on substantial piers which take up the earth movement. Here it is important to avoid the danger of making a pier which itself becomes a pendulum and so complicates the recorded motion. The pier ought therefore to be broad rather than high. Concrete I metre square imbedded in clay to a depth of I metre gives satisfactory results. In the case of a complete Galitzin installation the pier is enlarged so that all three pendulums may be carried on it.

The co-ordinates of the station latitude, longitude, and height above sea-level must be known or determined in some suitable way, and it then becomes important to determine the position of the geographical meridian. It is convenient to record the N.-S. and E.-W. components directly, and it is important to make the adjustment correct from time to time or to determine how far the pendulums have deviated from the true positions. We also require to know the initial direction of motion of the recording point, when the earth moves in a given direction. Thus it is convenient to arrange that a movement up the sheet corresponds to an earth movement to north or east, and it is useful to remember here that if a sudden movement of the earth occurs, say to north, the initial motion of the centre of gravity of the pendulum will appear to be towards the south. As confusion on this point has occasionally arisen by the notion of forces applied to the pendulum, it is well to recall that we are concerned not with forces, but with a prescribed motion given to the point of support in which the recording part of the apparatus participates, so that it is only the relative movement of the pendulum that is observed. It may be that the prescribed initial motion is complicated and not instantaneous, so that the record is then mixed up with 
instrumental terms. The initial kinematical result remains true, but whether we succeed in detecting the true apparent initial movement of the pendulum on the record is another matter.

Accurate time marks must be put on the records automatically, and the station thus requires a good clock and a knowledge of standard Greenwich Mean Time, so that the occurrence of events at different stations may be compared with an accuracy of one second. Absolute time is less important than the consideration that all stations should have the same time. The use of the wireless time signal promises the best solution of this problem.

A word with regard to photographic registration may not be out of place. If sharp traces are to be obtained only the highest quality of optical work is permissible. Mirrors must be optically plane and palladianized on the front surface, lenses must be properly corrected for spherical and chromatic aberration, and the use of thick plates of glass through which the light has to pass at a high angle must be avoided.

The number of seismographs that have at various times been devised is very large, but only a few of these have survived to practical use at the present time. We cannot attempt to discuss these obsolete forms, although a study of them will well repay anyone interested in the improvement of practical seismometry (for references see Milne, "Earthquakes").

We have already remarked that the rotations are not yet recorded although instruments for measuring tilting have been proposed. The bifilar pendulum of Darwin and Davison ("B. A. Reports," I 88I) and the klinograph of Schluiter ("Gött. Dissert.," I900) have not come into use, as they record other things besides tilting. Galitzin (Vorlesungen) has recently proposed to record tilting by the combination of two similar horizontal aperiodic pendulums at different heights working in opposition on a single galvanometer.

Thus if $\theta_{1}$ represents the motion of the lower pendulum we have

$$
\ddot{\theta_{1}}+2 \epsilon \theta_{1}+n^{2} \theta_{1}=-(\ddot{x}-g \psi) / l
$$

while if $\theta_{2}$ represents the motion of the precisely similar pendulum at a height $s$

$$
\ddot{\theta}_{2}+2 \epsilon \dot{\theta}_{2}+n^{2} \theta_{2}=-(\ddot{x}-g \psi+s \ddot{\psi}) / l
$$


hence

$$
\ddot{\theta}_{1}-\ddot{\theta}_{2}+2 \epsilon\left(\dot{\theta}_{1}-\dot{\theta}_{2}\right)+n^{2}\left(\theta_{1}-\theta_{2}\right)=s \ddot{\psi} / l
$$

so that the differential motion is quite independent of $x$ and depends only on $\psi$. Experiments at Pulkowa on an experimental table give very satisfactory results, but it remains to be seen how this arrangement does for continuous recording.

I am not aware that any experiments have been made with a view to recording rotation about the vertical, but the use of electromagnetic registration appears to offer a way of recording this on a large enough scale. Suppose that we have a heavy rod suspended by a vertical wire which passes through its centre of gravity so that the rod rests horizontally. If now the rod carries similar flat coils at its ends moving in strong magnetic fields, the coils being coupled through a galvanometer so as to assist each other when the rod rotates, the equation will then take the form

$$
\ddot{\theta}+2 \epsilon \dot{\theta}+n^{2} \theta=-\ddot{\chi}
$$

and the motion will be independent of $\ddot{x}$ and $\psi$.

We must remember that, however well we may be able to adjust apparatus to measure artificial rotations in the laboratory, for practical continuous recording of earth tilting we have to make sure that with slight secular changes of the constants the apparatus does not develop a tendency to record a part of the comparatively large values of $\ddot{x}$.

With regard to the recording of the linear displacements there still seems to be ample scope for the improvement of existing forms of apparatus. The ideal to aim at is the precise reproduction of the earth movement on a suitable scale of magnification. No instrument does this although some come nearer it than others. Thus consideration of the dimensions of a seismograph appears to me to merit more attention than has already been given. The following table shows the diversity of magnitudes in existing forms :-

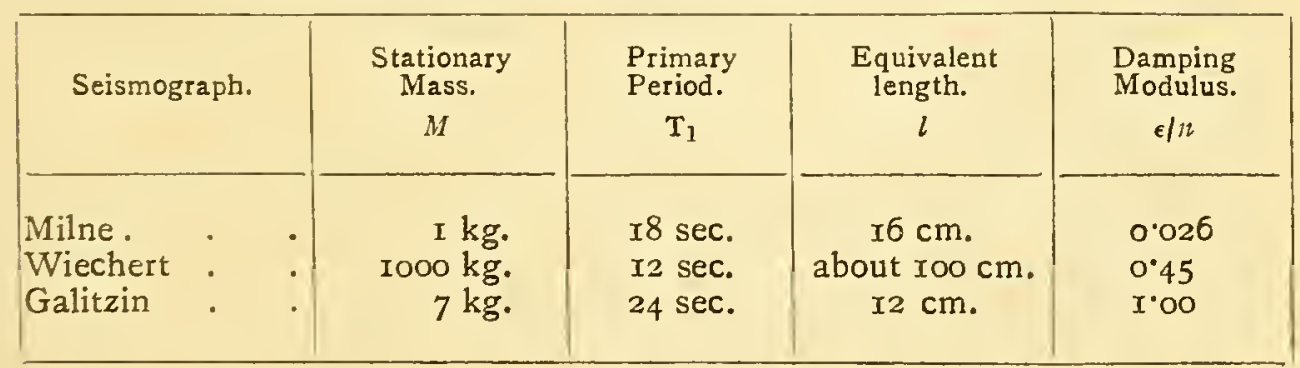


The statement sometimes made that the Omori Seismograph has a natural period of sixty seconds, I take to refer to the apparent period when friction of the recording system' is introduced. Personally I found the Omori became unstable with a natural period above $I 6^{s}$ and $I$ am convinced that there is no piece of physical apparatus of reasonable dimensions that could have a natural period of sixty seconds. Instability due to mechanical imperfection sets in long before this.

The differences in $M$ are very great and Wiechert even used I 7,000 kg. in his celebrated instrument at Göttingen. If we go back to the physical equation of motion of a pendulum, we see that except for any controlling spring action or -solid friction there is no point in using a large mass. On the contrary, if we admit the desirability of high damping we have everything to gain by using a small mass, as we then make $\epsilon$ larger without altering $n$ and $l$. There will be a practical limit to $M$ depending on very small spring action that might ordinarily be neglected, but I do not think the practical limit of small mass has yet been reached.

We have already commented on the advantage of high damping in removing the effect of instrumental vibration, and we must now consider more fully the question of aperiodicity. The mathematical advantage of ideal aperiodicity is to some extent discounted by the fact that it cannot be precisely maintained, and its practical advantage of freeing the record from instrumental periodicity might be also secured in an overdamped pendulum. But aperiodicity alone does not solve the problem of deducing the earth movement. If an aperiodic pendulum is given a sudden displacement it would still show I per cent of its initial displacement when $n t=6.64$ or if

$$
\mathrm{T}_{1}=\frac{2 \pi}{n}=24 \mathrm{sec} ., t=25 \mathrm{sec} \text {. }
$$

Now $t$ can only be reduced by increasing $\epsilon$ and we could then only retain aperiodicity by shortening the primary period. Further, if galvanometric registration is used, the record corresponding to a sudden displacement of the ground presents the appearance of a single wave.

Thus for certain types of movement such as occur in the 
earlier phases of a seismogram, instrumental terms have a pronounced influence in any case, but the interpretation of the record is greatly facilitated if we can depend on the rapid decay of the "free terms".

Under the influence of periodic waves the magnification is given by

$$
\frac{\mathrm{L}}{l} p^{2}\left\{\left(n^{2}-p^{2}\right)^{2}+4 \epsilon^{2} p^{2}\right\}^{-\frac{1}{2}}
$$

and in the case of an aperiodic pendulum this becomes

$$
\frac{\mathrm{L}}{l} \frac{p^{2}}{n^{2}+p^{2}} \text { or } \frac{\mathrm{L}}{l} \frac{\mathrm{T}_{1}{ }^{2}}{\mathrm{~T}_{1}{ }^{2}+\mathrm{T}^{2}} \text {. }
$$

Thus the magnification is dependent on the period of the impressed vibrations, and we can extend the range over which approximate uniformity is obtained only by an increase of the primary period $T_{1}$. But this means that if we use heavy damping we must be prepared to sacrifice true aperiodicity.

For rapid vibrations the magnification is $\mathrm{L} / l$ and for slow vibrations the magnification is

$$
\frac{L p^{2}}{\ln n^{2}} \text { or } \frac{L p^{2}}{g i} \text {. }
$$

We can thus increase the magnification for rapid vibrations by reducing $l$ and that for long waves by reducing $i$, and this might be done without any great change in $n$ or $2 \pi / T_{1}$ from the values at present attainable, say $T_{1}=$ twenty seconds. Now $l$ may be reduced by reducing the dimensions of the pendulum, and if $i$ is correspondingly reduced $T_{1}$ would not be altered. The reduction of dimensions would not greatly alter $\epsilon$, but the reduction of the mass would increase $\epsilon$ considerably.

The point I wish to put is this, that we have much to gain and little to lose by a substantial reduction in mass and length of the pendulums as at present used. To be definite it appears to be practically possible by the use of a fine quartz Zöllner suspension to make a pendulum in which $l$ is of the order I cm., M of the order I gram, which is highly damped ( $\epsilon$ of order say I), and which could be placed inside a vessel the size of an ordinary tumbler. With optical registration at a distance of 2 metres the magnification for rapid vibrations 
would be about 240 , or forty times that of the present Milne Seismograph.

Although no seismograph at present gives an exact reproduction of the earth movement in general, we ought not to regard the attainment of this as impracticable. The relation expressed by

$$
\ddot{y}+2 \epsilon \dot{y}+n^{2} y=-\lambda \ddot{x}
$$

is not the most general that may obtain between the impressed co-ordinate $x$ and the recorded co-ordinate $y$. The general form is

$$
\ddot{y}+2 \epsilon \dot{y}+n^{2} y=-\lambda\left(\ddot{x}+2 \epsilon_{1} \dot{x}+n_{1}^{2} x\right)
$$

and if it should prove possible to get a practical arrangement in which $n=n_{1}$ and $\epsilon=\epsilon_{1}$ the latter being great, we should then come very near to a precise reproduction of uniform magnification. 


\section{CHAPTER VI.}

THEORY OF A SOLID ISOTROPIC EARTH.

A COMPARISON of seismograms obtained at different stations suggests at once that we are concerned with mechanical effects propagated from the region in which the earthquake occurred.

We are thus led to inquire what is the nature of the effects propagated and to form a working theory as to the physical properties of the Earth, which will enable us to co-ordinate the observations.

At the present time the evidence in favour of a solid Earth is very great, but the alternative view that the interior of the Earth is fluid retarded for a considerable time the progress of seismological theory, which requires the Earth to possess the properties of an elastic solid.

As astronomical theory agrees with seismological in demanding a solid earth we accept this as a primary condition.

The simplest assumption we can make is that the physical properties of the Earth are uniform throughout, and although we shall find that seismology requires a modification of this assumption, yet many important features of a seismogram become intelligible on the basis of this simple hypothesis, and quantitatively the differences are not so great but that we may regard a uniform isotropic Earth as giving a good first approximation to the co-ordination of results. Accordingly it is instructive to begin by a consideration of the effects to be expected on this view, as it prepares us to make a first interpretation of a seismogram and to see on what lines the modification has to proceed.

The fundamental equations of motion of a uniform isotropic solid are so fully dealt with in treatises on elasticity (e.g. Love's 
"Theory of Elasticity") that the results are quoted here without proof.

If the independent variables are $x, y, z$, the Cartesian coordinates of a point, and $t$ the time, and the dependent variables are $u, v, w$, the components of displacement of a particle at $x, y, z$, then the equations are

$$
\begin{gathered}
\left(\rho \frac{\partial^{2}}{\partial t^{2}}-\mu \nabla^{2}\right)(u, v, w)=(\lambda+\mu)\left(\frac{\partial}{\partial x}, \frac{\partial}{\partial y}, \frac{\partial}{\partial z}\right) \theta \\
\theta=\frac{\partial u}{\partial x}+\frac{\partial v}{\partial y}+\frac{\partial w}{\partial z} \\
\rho=\text { the density }
\end{gathered}
$$

where

and $\lambda$ and $\mu$ are constants defining the elastic properties of the medium.

If $\theta \neq 0$ we get

$$
\rho \frac{\partial^{2}}{\partial t^{2}} \theta=(\lambda+2 \mu) \nabla^{2} \theta
$$

while if $\theta=0$ we have

with

$$
\begin{gathered}
\left(\rho \frac{\partial^{2}}{\partial t^{2}}-\mu \nabla^{2}\right)(u, v, w)=0 \\
\frac{\partial u}{\partial x}+\frac{\partial v}{\partial y}+\frac{\partial w}{\partial z}=0
\end{gathered}
$$

We thus find that the motion can be analysed into two types: (I) the longitudinal type $\theta \neq 0$ in which the velocity of propagation is $V_{1}=(\lambda+2 \mu)^{\frac{1}{2}} / \rho^{\frac{3}{2}}$ and the displacement is in the direction of propagation, and (2) the transversal type $\theta=0$ in which the velocity of propagation is $\mathrm{V}_{2}=\mu^{\frac{1}{2}} / \rho^{\frac{1}{2}}$ and the displacement is at right angles to the direction of propagation.

The components of stress at any point are in the usual notation

$$
\begin{aligned}
&\left(\mathrm{X}_{x}, \mathrm{Y}_{y}, \mathrm{Z}_{z}\right)=(\mathrm{I}, \mathrm{I}, \mathrm{I}) \lambda \theta+2 \mu\left(\frac{\partial}{\partial x}, \frac{\partial}{\partial y}, \frac{\partial}{\partial z}\right)(u, v, w) \\
& \mathrm{Y}_{z}=Z_{y}=\mu\left(\frac{\partial w}{\partial y}+\frac{\partial v}{\partial z}\right) \\
& \mathrm{Z}_{x}=\mathrm{X}_{z}=\mu\left(\frac{\partial u}{\partial z}+\frac{\partial w}{\partial x}\right) \\
& \mathrm{X}_{y}=\mathrm{Y}_{x}=\mu\left(\frac{\partial v}{\partial x}+\frac{\partial u}{\partial y}\right) .
\end{aligned}
$$

Although the effects of an earthquake observed at a distant station may persist even for several hours, we have cumulative 
evidence that the primary disturbance at the focus consists of a concentrated shock or limited series of shocks occurring within a very short time, a matter of some seconds. In any case we are certainly not concerned with unlimited trains of waves proceeding from the focus, so that our discussion must now proceed in the light of Stokes' "Dynamical Theory of Diffraction " (Collected Papers) in which he considers the effect of an arbitrary initial disturbance produced in the vicinity of a point.

In an unlimited medium the disturbance spreads in spherical shells from the origin. If the primary disturbance is of short duration, the effects observed at a point distant $r$ will be first a short disturbance at the time taken for the longitudinal waves of velocity $V_{1}$ to reach the point, then a period of quiescence followed by a second short disturbance when the transversal waves of velocity $\mathrm{V}_{2}\left(<\mathrm{V}_{1}\right)$ reach the point, after which the motion at $r$ ceases. The relative magnitudes of these effects depends not only on the distance $r$, but also on how the primary disturbance can be analysed into the two types, and in particular one or other may vanish. We have also to note that the effects are not the same at all points at distance $r$, but depend on the axis or axes of the constituents of the initial disturbance.

When we pass to the case of the Earth, we shall suppose in accordance with observation that the origin of disturbance is situated at a point comparatively near the surface of the earth. We may still expect that the seismogram obtained at a point on the earth's surface will, in general, be characterized by a pronounced movement corresponding to the arrival of the longitudinal disturbance, and by a pronounced movement when the transversal disturbance arrives, both of which have travelled by the brachistochronic path (in this case a straight line) from the focus to the station. These are accordingly indentified with the beginning of the first phase $\mathrm{P}$ and the second phase $\mathrm{S}$ of a seismogram.

From observations made at comparatively small distances $(<1000 \mathrm{~km}$.) from the focus, Zöppritz and Geiger find that $V_{1}=7.17 \mathrm{~km}$. per second, and $V_{2}=4^{\circ}$ or $\mathrm{km}$. per second, and 
these are adopted as the surface values. For greater distances we have to abandon the supposition that the velocities are constant throughout the earth, but this point we postpone to a later chapter and meanwhile retain the hypothesis of uniformity.

The boundary of the earth introduces many new features in the seismogram to be observed at a station, over and above those which we have mentioned and which will be referred to briefly as $\mathrm{P}$ and $\mathrm{S}$.

Following Huygens' principle, each point of the spherical disturbances $\left(V_{1}\right.$ and $\left.V_{2}\right)$ spreading out from the focus will, as it reaches the earth's surface, become a centre from which

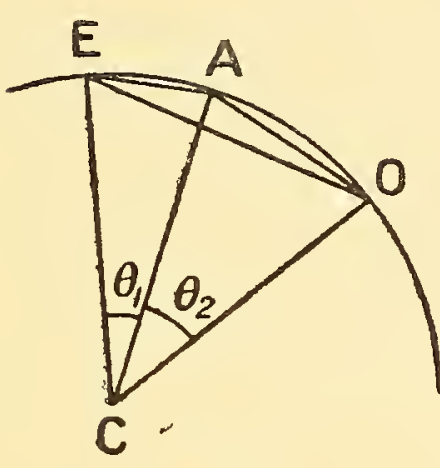

FIG. 9. spread two spherical disturbances $\left(V_{1}\right.$ and $V_{2}$ ), so that we have on the seismogram a whole series of diffraction effects in addition to $\mathrm{P}$ and $\mathrm{S}$.

Let $\mathrm{E}$ represent the earthquake focus supposed to be near the surface, $\mathrm{O}$ the station, and $\mathrm{C}$ the centre of the earth. Further, let the earth's radius be $R$ and the angular distance

$\mathrm{EO}$ be $\theta$. Then the arc

$$
\begin{aligned}
\mathrm{EO} & =\Delta=\mathrm{R} \theta \\
\text { and chord } \mathrm{EO} & =2 \mathrm{R} \sin \theta / 2 .
\end{aligned}
$$

The first effect at $\mathrm{O}$ is the beginning of the longitudinal phase $P$ at a time

$$
t_{1}=\frac{2 \mathrm{R}}{\mathrm{V}_{1}} \sin \frac{\theta}{2}
$$

Now consider the disturbance which travels as a longitudinal disturbance $V_{1}$ by the path EA and then as a diffracted longitudinal disturbance $V_{1}$ by the path $A O$. It reaches the station at a time

$$
t=\frac{2 \mathrm{R}}{\mathrm{V}_{1}}\left(\sin \frac{\theta_{1}}{2}+\sin \frac{\theta_{2}}{2}\right)=\frac{4 \mathrm{R}}{\mathrm{V}_{1}} \sin \frac{\theta}{4} \cos \left(\frac{\theta_{1}-\theta_{2}}{4}\right) .
$$

These disturbances start immediately after $\mathrm{P}$ and arrive at later and later instants for $\theta_{1}>$ or $<\theta_{2}$ until they culminate in the brachistochronic path of maximum time, which is also that of regular reflexion, when $\theta_{1}=\theta_{2}$. 
We may thus expect a pronounced effect at a time

$$
\frac{4 \mathrm{R}}{\mathrm{V}_{1}} \sin \frac{\theta}{4}
$$

It is a longitudinal effect and may be identified with Wiechert's first reflected effect $\mathrm{PR}_{1}$. In practice it is often more pronounced than $P$ in the case of distant earthquakes, and is then of considerable value in determining the position of the earthquake region. The argument may be extended to further subdivisions of the arc EO.

The second or transverse phase begins with $\mathrm{S}$ at a time

$$
t_{2}=\frac{2 \mathrm{R}}{\mathrm{V}_{2}} \sin \frac{\theta}{2}
$$

Next consider the longitudinal effect which travels by EA with velocity $V_{1}$ and is diffracted as a transversal effect along AO with velocity $\mathrm{V}_{2}$. The time of arrival is

$$
t=\frac{2 \mathrm{R} \sin \theta_{1} / 2}{\mathrm{~V}_{1}}+\frac{2 \mathrm{R} \sin \theta_{2} / 2}{\mathrm{~V}_{2}}
$$

For different positions of $\mathrm{A}$ these effects, which begin immediately after $\mathrm{P}$, arrive at later and later instants and culminate in the brachistochronic path of maximum time which is that of regular reflexion determined by

$$
\cos \theta_{1} / 2=\frac{\mathrm{V}_{1}}{\mathrm{~V}_{2}} \cos \theta_{2} / 2
$$

But here an interesting point arises. Since $\theta_{1}+\theta_{2}=\theta$ we get

$$
\tan \frac{\theta_{1}}{2}=\left(\frac{\mathrm{V}_{2}}{\mathrm{~V}_{1}}-\cos \frac{\theta}{2}\right) / \sin \theta / 2
$$

and thus we cannot get a real positive value of $\theta_{1}$ unless $\cos \theta / 2$ is $<\mathrm{V}_{2} / \mathrm{V}_{1}$. This implies that if $\theta$ is less than the value given by $\cos \theta / 2=V_{2} / V_{1}$ the diffracted effects continue up to $S$ without any pronounced movement, but if $\theta$ exceeds this critical value the diffracted effects may be expected to culminate in a maximum transversal effect at a time

$$
t_{3}=\frac{2 \mathrm{R}}{\mathrm{V}_{2}} \frac{\sin \theta / 2}{\cos \theta_{1} / 2}
$$

which is later than the arrival of $S$. This point is of real practical importance. With the values of $V_{1}$ and $V_{2}$ as given, 
we get $\theta=\mathrm{IIO}^{\circ}$ nearly or $\Delta=$ about $\mathrm{I} 2,000 \mathrm{~km}$. Now it has been observed that special difficulty attaches to the identification of $\mathrm{S}$ just when $\Delta$ is about $\mathrm{I} 2,000 \mathrm{~km}$. Thus with an earthquake in the northern Philippines which are about I I,Ooo $\mathrm{km}$. from this country $\mathrm{S}$ usually comes out very clearly, while in the case of an earthquake in the Caroline Islands about $\mathrm{I} 2,000 \mathrm{~km}$. from us $\mathrm{S}$ is most indistinct and the tendency is to put it rather late. The result we have obtained throws some light on the matter.

We may have a disturbance which starts as transversal with velocity $V_{2}$ along EA and then proceeds as longitudinal with velocity $V_{1}$ along AO. Here again we cannot expect any pronounced effect unless $\theta$ is greater than the value given by $\cos \theta / 2=\mathrm{V}_{2} / \mathrm{V}_{1}$.

Lastly we have the disturbances that traverse the whole path with velocity $V_{2}$. These start after $S$ and culminate in a maximum when

$$
t=\frac{4 \mathrm{R}}{\mathrm{V}_{2}} \sin \frac{\theta}{4}
$$

and this we may identify with Wiechert's $\mathrm{SR}_{1}$.

We must of course add to the cases indicated, the disturbances that travel to the station by the opposite side of the earth. They may be considered by the method already used, and we shall point out only the $\mathrm{PR}_{1}$ which reaches the station by the longer path. It arrives when

$$
t=\frac{4 \mathrm{R}}{\mathrm{V}_{1}} \cos \theta / 4
$$

and will thus be later than $\mathrm{S}$ unless

$$
\sin \theta / 4>\frac{V_{2}}{V_{1}} \text {. }
$$

The critical value is $\theta=$ about $140^{\circ}$ or $\Delta=$ about $\mathrm{I} 5,500 \mathrm{~km}$. Thus we have here another critical value tending to indistinctness of the second phase $\mathrm{S}$.

There appears to be no reason why we should not also have diffracted effects in which $\theta_{1}$ is negative.

Let us now consider the problem of regular reflexion when a disturbance of either type is incident at a point on the earth's surface. 
First-Plane longitudinal disturbance incident. The displacement $\xi_{1}, \eta_{1}, \zeta_{1}$, due to the incident disturbance may be written

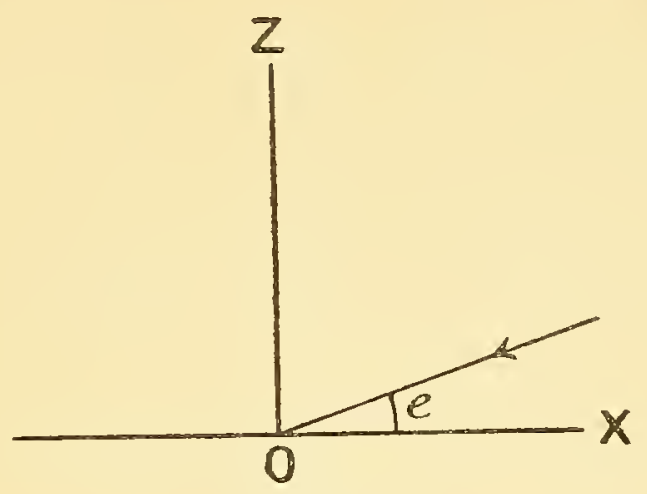

FIG. IO.

$$
\left(\xi_{1}, \eta_{1}, \zeta_{1}\right)=-\mathrm{A}(\cos e, \mathrm{o}, \sin e) f\left(t+\frac{x \cos e+z \sin e}{\mathrm{~V}_{1}}\right) .
$$

This gives rise to a reflected longitudinal disturbance expressed by

$$
\left(\xi_{2}, \eta_{2}, \zeta_{2}\right)=-\mathrm{A}_{2}(\cos , e, \mathrm{o},-\sin e) f\left(t+\frac{x \cos e-z \sin e}{\mathrm{~V}_{1}}\right)
$$

and a reflected transversal disturbance

$$
\left(\xi_{3}, \eta_{3}, \zeta_{3}\right)=\mathrm{A}_{3}\left(\sin e^{\prime}, \mathrm{o}, \cos e^{\prime}\right) f\left(t+\frac{x \cos e^{\prime}-z \sin e^{\prime}}{\mathrm{V}_{2}}\right) .
$$

As the surface must be free from traction we have

$$
Z_{z}=Z_{y}=Z_{x}=0 \text {. }
$$

This leads to the following relations

$$
\begin{aligned}
& \mathrm{A}-\mathrm{A}_{2}=\mu \mathrm{A}_{3} \cos 2 e^{\prime} / \sin 2 e \\
& \mathrm{~A}+\mathrm{A}_{2}=\frac{\mathrm{I}}{\mu} \mathrm{A}_{3} \sin 2 e^{\prime} / \cos 2 e^{\prime}
\end{aligned}
$$

where $\mu \cos e^{\prime}=\cos e$ and $\mu=V_{1} / V_{2}$

Thus the apparent direction of motion of the ground is given by $\zeta / \xi=\tan \bar{e}=-\cos 2 e^{\prime}$

Hence

$$
\cos e=\frac{\mathrm{V}_{1}}{\mathrm{~V}_{2}}\left(\frac{\mathrm{I}-\sin \tilde{e}}{2}\right)^{\frac{1}{2}} .
$$

This relation is of considerable practical importance.

We have to note that for $e=0$ the resulting motion of the ground is nil whatever A may be. It is thus impossible to have a longitudinal disturbance in which the direction of displacement is parallel to the surface propagated along the surface. 
Second-Incident transversal disturbance, displacement perpendicular to the plane of the paper.

In this case it is found that no longitudinal disturbance arises and that the incident effect is reflected as a transversal effect without change. The motion of the ground is entirely horizontal and equals twice that of the incident disturbance. In this case it is possible to have a tranversal disturbance propagated parallel to the surface, the displacement being at right angles to the direction of propagation and parallel to the surface.

Third-Incident transversal disturbance, displacement in the plane of the paper.

We assume as the incident disturbance

$$
\left(\xi_{1}, \eta_{1}, \zeta_{1}\right)=\mathrm{A}(-\sin e, 0, \cos e) f\left(t+\frac{x \cos e+z \sin e}{\mathrm{~V}_{2}}\right)
$$

which gives rise to the reflected transversal effect

$$
\left(\xi_{2}, \eta_{2}, \zeta_{2}\right)=\mathrm{A}_{2}(\sin e, 0, \cos e) f\left(t+\frac{x \cos e-z \sin e}{\mathrm{~V}_{2}}\right)
$$

and the reflected longitudinal effect

$$
\left(\xi_{3}, \eta_{3}, \zeta_{3}\right)=\mathrm{A}_{3}\left(-\cos e^{\prime}, \mathrm{O}, \sin e^{\prime}\right) f\left(t+\frac{x \cos e^{\prime}-z \sin e^{\prime}}{\mathrm{V}_{1}}\right) .
$$

Application of the surface condition gives

$$
\begin{aligned}
& A-A_{2}=-\mu A_{3} \cos 2 e / \sin 2 e \\
& A+A_{2}=-\frac{I}{\mu} A_{3} \sin 2 e^{\prime} / \cos 2 e
\end{aligned}
$$

where $\mu \cos e=\cos e^{\prime}$ and $\mu=V_{1} / V_{2}$

the apparent direction of motion of the ground is given by

$$
\zeta / \xi=\tan \bar{e}=\frac{2}{\mu} \sin e^{\prime} \cos e / \cos 2 e .
$$

This holds as long as $\cos e \ngtr \mathrm{I} / \mu$, but when $e$ is less than the value given by $\cos e=\mathrm{I} / \mu, e^{\prime}$ is imaginary, and complex values have to be assumed for $A_{2}$ and $A_{3}$. The result is a reflexion of transversal disturbance with a change of phase while there exists a type of longitudinal disturbance in which the amplitude diminishes rapidly away from the surface, but which cannot in any true sense be regarded as propagated as there is no real wave front.

It is important to note that at the critical angle the vertical 
motion of the ground is zero, and only horizontal motion in the plane of incidence remains. On the other hand when $e=45^{\circ}$ the horizontal motion is zero and only vertical motion remains. Further when $e=0$ the motion of the ground is zero whatever A may be, and thus transversal disturbance in which the displacement is perpendicular to the ground cannot be propagated parallel to the surface.

Our discussion, which has proceeded on elementary lines, is particularly useful in showing how difficulty arises in detecting $\mathrm{S}$ at considerable distances owing to interference with other maxima, or it may be the actual vanishing of the horizontal movement. It has indeed sometimes been asserted that $\mathrm{S}$ never reaches beyond a certain distance, and to explain this an impenetrable core of the earth has been assumed. We see that no such hypothesis is at all necessary to explain the observations.

A complete discussion which shall take account of the magnitude of the diffracted effects as well as of their time of arrival even for a simple type of initial disturbance would, I believe, be a valuable contribution to seismological theory, and in particular I should hope that it would throw some light on the origin of a class of waves we have still to consider.

We have observed that it is impossible to propagate along a plane boundary either longitudinal waves with displacement parallel to the surface or transversal waves with displacement perpendicular to the surface. But by combining two types in which the direction of the wave front is expressed by imaginary angles, Lord Rayleigh (Collected Papers) has shown that the surface conditions may be satisfied and that a system of waves, in which the amplitude diminishes exponentially from the surface, appears to advance parallel to the surface.

If Poisson's ratio for the material $=\mathrm{I} / 4$ or $\mathrm{V}_{1}{ }^{2}=3 \mathrm{~V}_{2}{ }^{2}$ which is very nearly the case for the earth's surface, Lord Rayleigh finds that we can have a system of waves in which the displacement $\xi$ parallel to the surface and in the direction of apparent propagation is given by

and the vertical motion is

$$
\xi=\left(e^{-r z}-5773 e^{-s z}\right) \sin (p t+f x)
$$


where

$$
\zeta=\left(\cdot 8475 e^{-1 z}-1 \cdot 4679 e^{-s z}\right) \cos (p t+f x)
$$

and

$$
r=8475 f \quad s=3933 f
$$

Thus at the surface

$$
p \mid f=\mathrm{V}=9194 \mathrm{~V}_{2} \text {. }
$$

$$
\begin{aligned}
& \xi_{0}=4227 \sin (p t+f x) \\
& \xi_{0}=-.6204 \cos (p t+f x)
\end{aligned}
$$

so that the vertical motion is $\mathrm{I} 47 \mathrm{t}$ times the horizontal motion and the apparent velocity of propagation is less than the velocity $V_{2}$. Similar waves are possible at any plane boundary of two media.

It has been sought to identify these waves with the long waves that make their appearance in a sesimogram after the second phase $\mathrm{S}$. We shall postpone the discussion to the next chapter, but meanwhile it is important to observe that we must not regard Rayleigh waves as propagated in the same sense as the longitudinal and transversal types in the medium. We do not know the conditions that determine a surface separating an undisturbed portion of the medium from a portion influenced by these waves, and since the equations require the whole medium to be in motion it is difficult to specify the manner in which they can be originated.

We have so far regarded the focus as being situated at a - point on the earth's surface. But the focus of a large tectonic

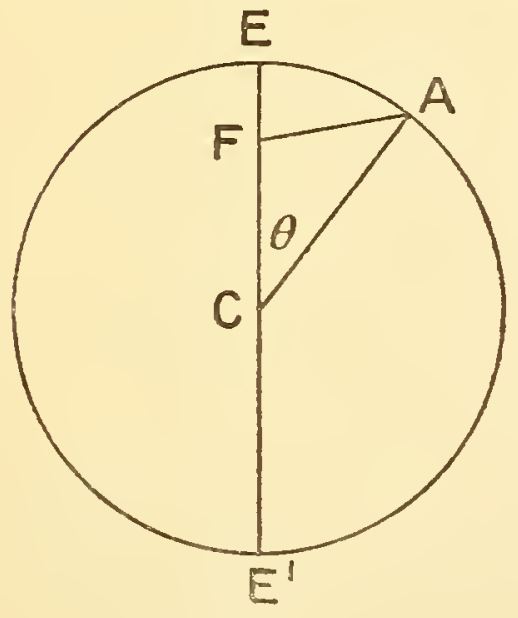

FIG. II. earthquake is probably situated at some depth of the order of $\mathrm{Io} \mathrm{km}$. Indeed it would seem to be the case that if the focus is very near the surface the effects are stifled within a very short distance, and that it requires a fair depth of the focus in order that the earth may be given, so to speak, a good shake which will be experienced at remote points.

A most important influence of a finite depth of focus is the manner in which it modifies the so-called angle of emergence $\bar{e}$-at the station. If $h=$ the depth of the focus we get 


$$
\cos e=\frac{\mathrm{R}-h}{\mathrm{R}} \sin \mathrm{AFC}
$$

so that instead of $e$ starting at o for $\theta=0$ and increasing $h$ to $\pi / 2$ for $\| \theta \pi, e$ begins at $\pi / 2$ for $\theta=0$, reaches a minimum when $\mathrm{AFC}=\pi / 2$, and then increases to $\pi / 2$ for $\theta=\pi$. The minimum is given by

$$
\cos e=\cos \theta=\frac{\mathrm{R}-h}{\mathrm{R}}, \text { or } \sin \theta / 2=\left(\frac{1}{2} \frac{h}{\mathrm{R}}\right)^{\frac{1}{2}} .
$$

This point is important in attempting to determine $h$ from observations. As an example if $h=10 \mathrm{~km}$., $\mathrm{R}=6370 \mathrm{~km}$., we get $\theta=3^{\circ} \mathrm{I} 2^{\prime}, \Delta=356 \mathrm{~km}$, and the corresponding apparent angle of emergence $\bar{e}=22^{\circ}$. But for $\Delta=1000 \mathrm{~km}$. the error in $e$ made by supposing $F$ to coincide with $\mathrm{E}$ would only be about $\frac{1}{2}^{\circ}$. 


\section{CHAPTER VII.}

\section{INTERPRETATION OF SEISMOGRAMS AND THE INTERIOR OF THE EARTH.}

IT may be remarked of most seismograms that, on first acquaintance, it is difficult to see the wood for the trees. Only by experience and study is it possible to disentangle those effects that are characteristic and essential from those that are accidental. Not only so, but we must keep in view that any seismogram is influenced by the particular instrument from which it is obtained. A record from an undamped instrument is for instance dominated throughout by instrumental periodicity. Heavily damped instruments, on the other hand, agree wonderfully well in presenting the same general features, and it is chiefly as regards relative magnitude of effect in different parts of the seismogram that they differ. The speed of registration also plays an important part, as movements that are resolved with high speeds get crushed together at lower speeds.

It is unfortunate that general statements with regard to the character of seismic waves have obtained credence, which are really dependent on one particular instrument. I am thus diffident about giving a general description of a seismogram that may convey a false impression, but as some description must be given it may be well to state that $I$ have in view heavily damped seismographs in general, and in particular Galitzin's aperiodic pendulums with galvanometric registration at a high speed. I would add that anyone who desires to work at any of the theoretical problems awaiting solution will do well to study actual seismograms for himself and not accept descriptions made by other people.

We shall suppose that records of the horizontal motion $(X, Y)$ and of the vertical motion $(Z)$ are available, and in 
practice it is desirable that the fundamental constants of all three instruments should be precisely the same.

The first phase (undae primae) is initiated by $\mathrm{P}$ either as a sharp impulse (impetus) or rapid succession of impulses, or by a more gradual development (emersio). This lasts a few seconds, and is interpreted as the arrival of longitudinal waves. In many cases $\mathrm{P}$ is more pronounced in $\mathrm{Z}$ than in (XY). $\mathrm{P}$ is succeeded by a series of smaller movements of a very irregular character, with turning points sharply marked, at intervals of a few seconds. There is in general a marked absence of periodicity or motion of a sinusoidal nature. We do, however, sometimes find minute movements with a period of about I second, and I have seen an instance (earthquake in Yap) where $P$ started with a few waves of small amplitude of a distinctly sinusoidal nature. Such cases are, however, rare.

During the first phase we have some outstanding sharp movements. If these happen to be the P's of subsequent shocks they will be confirmed by the later part of the seismogram. They may, however, be the reflected effects $P R$, etc., corresponding to $\mathrm{P}$. I have already mentioned that with earthquakes in the Philippines $\mathrm{PR}_{1}$, which arrives here about four minutes after $P$, is usually much larger than $P$.

After the first phase, which lasts for a time depending on the distance from focus to station, the seismogram changes its type. There is as a rule a large movement denoted by $\mathrm{S}$ which initiates the second phase (undae secundae). Its incidence is less sharply marked than $\mathrm{P}$ and it is sometimes very indistinct. It is clearer in (XY) than in $\mathrm{Z}$. S is interpreted as the arrival of the transversal waves. Following $S$ the movements are again very irregular. They are larger than those occurring between $\mathrm{P}$ and $\mathrm{S}$, and occur at longer intervals. The turning-points are rounded, and occasionally give a suggestion of sinusoidal movement. During this phase we may have outstanding movements which may be the S's of subsequent shocks or the reflexions of $\mathrm{P}$ and $\mathrm{S}$. For distances $>$ I $1000 \mathrm{~km}$. it becomes difficult to say precisely when the second phase starts, and we have explained in the preceding chapter how this probably arises. 
The second phase lasts for a time depending on the distance, and then the whole appearance of the seismogram changes and assumes a strongly periodic and sinusoidal character. The point at which the change takes place is only rarely sharply marked and is not characterized by a large movement such as we have with $\mathrm{P}$ and $\mathrm{S}$. This phase (undae longae) is initiated by L. For distances not less than $2000 \mathrm{~km}$. the general appearance of this phase is marked by first a few waves of period about 20 seconds, gradually increasing in amplitude and looking as if they had been drawn with a shaking hand, then a rapid development of extremely smooth waves of rather shorter period which reach a maximum amplitude, subside, pass through a succession of maxima before merging into the tail of the earthquake or Coda.

For short distances, however, this description does not hold good. L succeeds S very quickly, shorter periods of about I 2 seconds prevail, and the duration of the whole phase becomes very short.

These remarks apply as a whole to $(X Y)$ and $Z$; but, as a rule, the development of this phase in $Z$ comes rather later than in $(X Y)$.

Following the maximal or long wave phase we have the Coda. The amplitudes are now small and the movements are somewhat irregular and lacking in smoothness. Still the motion here is on the whole periodic and sinusoidal (about I 2 seconds).

If the earthquake is a very large one, we may after about $2 \frac{1}{2}$ hours observe the arrival of long waves that have travelled by the opposite side of the earth. In this way Galitzin has found from the records of the great Messina earthquake of December, I908, that the long waves travel round the earth with a surface velocity of $3.53 \mathrm{~km}$. per second, which agrees well with the theoretical value for Rayleigh waves, viz. $0.9 \mathrm{I} 9 \times 4^{\circ} \mathrm{OI}=3.69 \mathrm{~km}$. per second.

The view that $\mathrm{P}$ and $\mathrm{S}$ represent the arrival of the longitudinal and transversal waves that have travelled by brachistochronic paths from the focus to the station may be accepted without much question. The difficulty that attaches to the 
interpretation of the first and second phases is that of the origin of the irregular movements that follow on $\mathrm{P}$ and $\mathrm{S}$. These may in some measure arise from subsidiary shocks either at the primary focus or at other points, and I have pointed out that in a uniform earth we have a diffraction effect due to the surface. This in itself is, however, insufficient, and the facts obtain an obvious explanation in the multiple diffraction of the primary disturbance that must go on in the heterogeneous mass of rock that constitutes the earth's crust. There will thus be not only one principal, but also many subsidiary brachistochronic paths from the focus to the station.

The suggestion that dispersion analogous to optical dispersion may be called in to explain the asserted oscillatory movement in the first and second phases may be dismissed as not required, since heavily damped seismographs show that there is no general oscillation to explain, but only a highly irregular succession of impulses. The influence of dispersion is shown in the rounding of turning-points, so that it is only a slightly modifying influence and not a determining cause.

This argument is not affected by the minute vibrations of period about I second that sometimes appear after $\mathrm{P}$ on both Wiechert's and Galitzin's instruments. They are only shown when the earthquake is very great or the station sufficiently near the focus, and are thus accidental and not essential. Wiechert's suggestion (see Wiechert and Zöppritz " Ueber Erdbeben Wellen Gött. Nach.," I 907) that they represent a natural vibration of a layer of rock seems to be the only explanation available.

We have next to consider the long waves. We have already remarked that they are found by measurement to travel round the earth's surface with a general speed agreeing closely with that of Rayleigh waves. But the long wave phase is a complex phenomenon, and the fact that the waves are strongly periodic (mainly 12 -second and 20-second periods) presents considerable difficulty when we remember that the primary disturbance is an impulse.

With regard to the long wave phase, it has been asserted that the first portion consists of waves in which the displace- 
ment is entirely horizontal and at right angles to the direction of propagation, and that there follows the maximum movement in which there is horizontal movement in the direction of propagation along with vertical motion. This is only very roughly true. The seismogram reproduced, Plate I I, is a case in which the first portion of the long wave phase gives horizontal motion in the direction of propagation, while in the following maximal phase the horizontal motion is at right angles to the direction of propagation. What shall we say of cases where horizontal motion transverse to the direction of propagation is associated with pronounced vertical motion, or where horizontal motion in the direction of propagation occurs with little or no vertical motion?

No combination of transverse waves of purely horizontal displacement (velocity $\mathrm{V}_{2}$ ) and of Rayleigh waves (velocity $\cdot 92 V_{2}$ ) will explain these facts, which, it appears to me, can only be met by supposing that the long wave phase is complicated by effects arising from reflexion backwards and forwards between the Earth's surface and a layer of discontinuity at some depth.

Wiechert ("Ueber Erdbebenwellen," 1.c.) introduced the hypothesis of such a crust resting on a sheet of plastic material (magma). So far as such a crust provides by its natural vibration a means of explaining the dominant period of the long waves (say 20 seconds) we may agree; although the argument that the thickness of the layer is half the wave length of the dominant waves, and thus about $35 \mathrm{~km}$., hardly applies to Rayleigh waves; $40 \mathrm{~km}$., however, as the half wave length of purely transversal waves travelling across the layer would give the 20 seconds period, and also about I 2 seconds for longitudinal waves travelling across the layer. But the assumption of a plastic sheet, which would hardly be accepted on astromonical grounds, would not serve to contain the long waves within the layer without at the same time confining the first and second phase movements, which we have to admit penetrate the whole Earth.

At present we know nothing as to whether these long waves diminish in amplitude as the depth increases, nor does it appear to me necessary to suppose that they do not pene- 
trate beneath the crust. What we do know is that there is a shell of radiation spreading from the focus, within which there is disturbance and beyond which there is none.

In this connexion it is worth while to remember that the long waves in a seismogram suggest an importance out of all proportion to their physical effect. For example in the Galitzin Seismograph (primary period $24^{s}$ ) we should have to divide the apparent amplitude of a vibration $20^{s}$ period by about $S$ in order to compare with the apparent amplitude of a vibration of $\mathrm{I}^{s}$ period, and if further we remember that to compare the accelerating effects we should have to divide again by 400 , we find that the long waves dwindle very much in their physical importance.

This entirely agrees with Wiechert's remark that the longwave phase, interesting as it is, is a residual phenomenon. Neverthless the elucidation of the Long-wave phase and the Coda is highly important on account of the information it promises to afford as to the crust of the Earth, and here it seems probable that seismic dispersion may play a very important part.

We shall next suppose that the times of incidence of $\mathrm{P}, \mathrm{S}$, and $\mathrm{L}$ have been determined at the station for a well-defined earthquake, and that similar determinations have been made at a number of stations distributed over the earth. Further, we shall suppose that by one or other of the methods to be described in the next chapter, the position of the focus and the time of occurrence has been ascertained. We are then in a position to set out on a diagram the time taken for P, S, and $\mathrm{L}$ to travel from the focus as a function of $\Delta$ and $h$. The curve so obtained may be called a time curve (Laufzeit kurve). For theoretical purposes it is, however, convenient to correct the curve to what we should have got had $h$ been 0 , and we then obtain a curve expressed by $\mathrm{T}=f(\Delta)$. The general character of the mean results so obtained by Zöppritz and Geiger from several well-defined earthquakes (Gött. Nach., 1907) are shown in Plate 5, and the values obtained by interpolation are given in the table, p. 54 . 


\begin{tabular}{|c|c|c|c|c|c|c|c|}
\hline \multirow[b]{2}{*}{ in kilometres. } & \multirow[b]{2}{*}{$\underset{\text { in seconds. }}{\mathrm{P}}$} & \multirow[b]{2}{*}{$\stackrel{S}{S}$ in seconds. } & \multirow{2}{*}{\multicolumn{2}{|c|}{$\theta / 2}}$. & \multicolumn{3}{|c|}{ For $\mathrm{P}$. } \\
\hline & & & & & $\begin{array}{c}e \\
\text { from } \\
\text { time curve. }\end{array}$ & $\begin{array}{c}\bar{e} \\
\text { computed. }\end{array}$ & $\begin{array}{c}\bar{e} \\
\text { observed a } \\
\text { Pulkowa. }\end{array}$ \\
\hline 0 & 0 & 0 & $0^{\circ}$ & $o^{\prime}$ & $0^{\circ}$ & $22^{\circ}$ & \\
\hline 500 & 69 & I 24 & $2^{\circ}$ & I5' & II & 23 & \\
\hline I, OOO & 136 & 244 & & & $2 I$ & 27 & \\
\hline $\mathrm{I}, 500$ & I99 & $35^{6}$ & & 45 & 30 & 32 & \\
\hline 2,000 & 257 & 460 & 9 & 0 & 37 & 37 & \\
\hline 2,500 & 3 IO & 555 & II I & $I_{4}$ & 44 & 42 & $48^{\circ}$ \\
\hline 3,000 & $35^{8}$ & $64 \mathrm{I}$ & 132 & 29 & 49 & 47 & 44 \\
\hline 3,500 & 402 & 719 & I5 & 44 & 53 & $5^{2}$ & 43 \\
\hline 4,000 & $44^{2}$ & 789 & I7 5 & 59 & 57 & 54 & 42 \\
\hline 4,500 & 478 & 854 & 20 & $\mathrm{I}_{4}$ & 60 & $5^{8}$ & 43 \\
\hline 5,000 & $5^{12}$ & $9 \mathrm{I} 3$ & 22 & 29 & 63 & 60 & 44 \\
\hline 5,500 & 542 & $97 \mathrm{I}$ & 244 & 44 & 65 & 62 & 46 \\
\hline 6,000 & 572 & $\mathbf{I}, 028$ & 265 & 59 & 65 & 62 & 48 \\
\hline 6,500 & 601 & $\mathrm{I}, 084$ & 29 I & $I_{4}$ & 65 & 63 & $5 I$ \\
\hline 7,000 & $63 I$ & $\mathrm{I}, \mathrm{I}_{4} \mathrm{O}$ & $3^{I}=$ & 29 & 65 & 63 & 54 \\
\hline 7,500 & 660 & I,I94 & 334 & 43 & 66 & 63 & $5^{8}$ \\
\hline 8,000 & 688 & $\mathrm{I}, 249$ & 35 & 58 & 66 & 64 & 62 \\
\hline 8,500 & 716 & $I, 30 I$ & $3^{8}$ & I3 & 67 & 64 & 65 \\
\hline 9,000 & 743 & $\mathrm{I}, 354$ & 402 & 28 & 67 & 65 & 67 \\
\hline 9,500 & 769 & $\mathrm{I}, 404$ & $4^{2} 4$ & 43 & 68 & 66 & 68 \\
\hline Io,ooo & 795 & $\mathrm{I}, 453$ & 445 & 58 & 69 & 67 & 70 \\
\hline Io, 500 & 820 & $I, 500$ & 47 & I3 & 70 & 67 & $7 I$ \\
\hline II, OOO & 844 & $\mathrm{I}, 545$ & 492 & 28 & 70 & 68 & 72 \\
\hline II, 500 & 867 & $\mathrm{I}, 588$ & 5 I & 43 & $7 I$ & 69 & $7^{2}$ \\
\hline I 2,000 & 888 & $I, 629$ & 53 & 58 & 72 & 70 & 73 \\
\hline I2, 500 & 909 & $\mathrm{I}, 668$ & $5^{6}$ & I2 & 73 & $7^{I}$ & 73 \\
\hline 13,000 & 929 & 1,705 & 58 & 27 & 74 & $7^{2}$ & 74 \\
\hline
\end{tabular}

Let $\mathrm{EA}$ and $\mathrm{EB}$, fig. I2, represent neighbouring paths, then

$$
\cos e=\frac{\mathrm{BC}}{\mathrm{AB}}=\mathrm{V} \frac{d \mathrm{~T}}{d \Delta}
$$

where $\mathrm{V}$ is the corresponding velocity of the wave at the surface. This importarit result, which applies to both $\mathrm{P}$ and $\mathrm{S}$

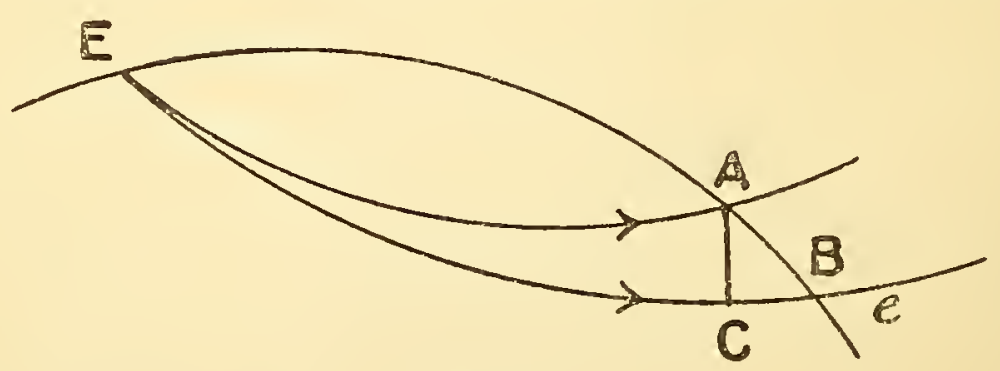

FIG. I2.

whatever be the path, is of course meaningless as applied to L. Since $V_{1}$ and $V_{2}$ are known we may from the time curves 


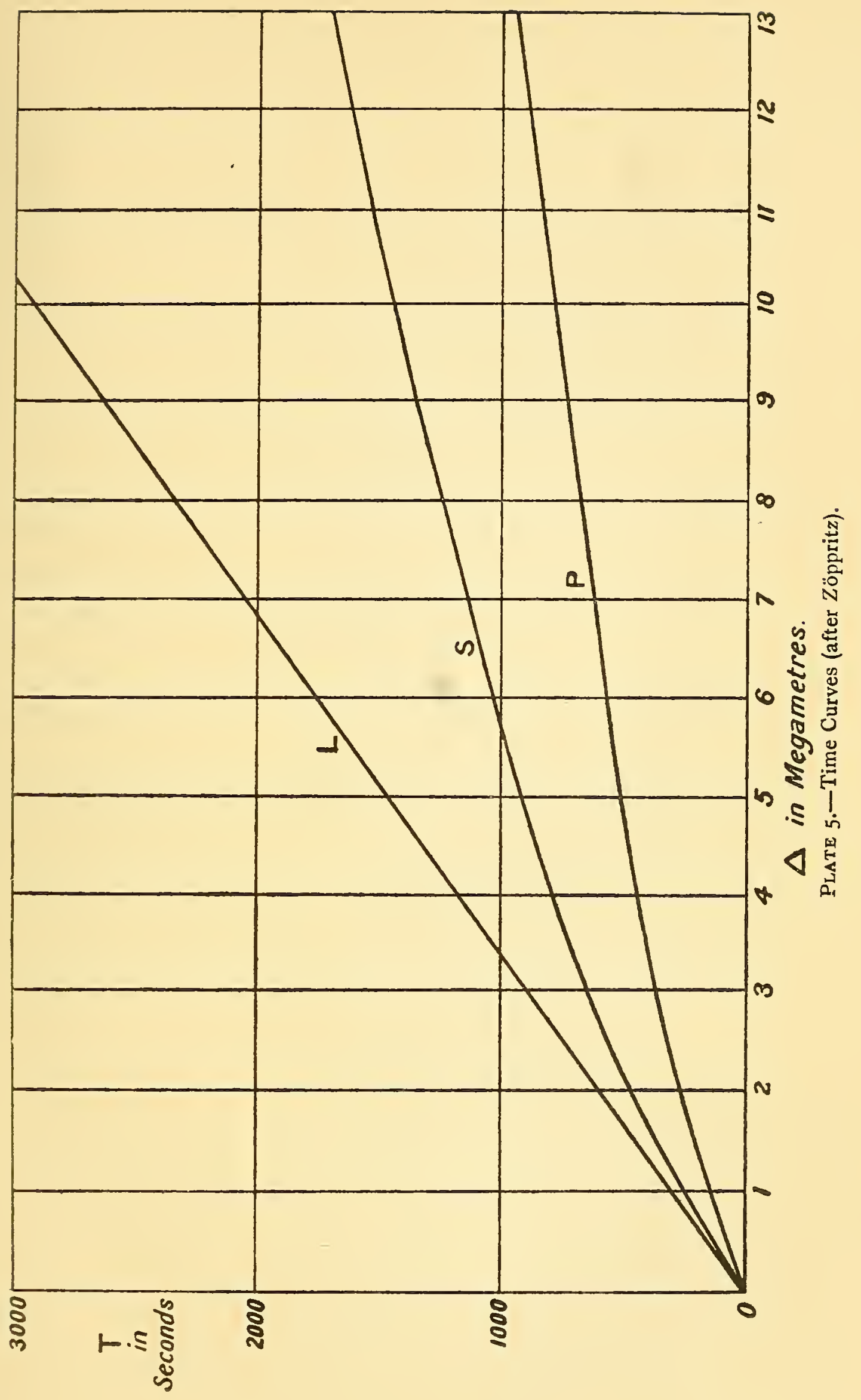



determine the corresponding angle $e$. For the longitudinal effect $\mathrm{P}$ we have

and we also have

$$
\cos e=\mathrm{V}_{1} \frac{d \mathrm{~T}_{1}}{d \Delta}
$$

$$
\cos e=\frac{\mathrm{V}_{1}}{\mathrm{~V}_{2}}\left(\frac{\mathrm{I}-\sin \bar{e}}{2}\right)^{\frac{1}{2}}
$$

where $\bar{e}$ is the apparent angle of emergence.

Now if the rays travel in a straight line from $\mathrm{E}$ to $\mathrm{A}$ the angle of emergence $\epsilon$ would be simply $\Delta / 2 \mathrm{R}=\theta / 2$.

The table, page 54 , shows at once that as we proceed to increasing distances the value of $e$ obtained from the time curve is much greater than the corresponding value of $\theta / 2$. Thus the rays dip more deeply into the earth than does the straight line from focus to station. The rays must on the whole be concave towards the surface, and we have now to abandon the hypothesis that the earth is uniform, and instead to assume that the velocity of propagation depends on the depth. Accordingly the next step is to suppose that the earth is made up of concentric uniform spherical shells, but that the velocity $v$ varies as a function of $r$ the radius of the shell. On this hypothesis the brachistochronic paths are still plane curves in planes containing the focus, Earth's centre, and the station, but are now curved, each curve being characterized by the wellknown equation $p / v=c$ (a constant) where $p$ is the perpendicular from the centre of the Earth on the tangent to the curve at any point. From the values at the surface we get

$$
p / v=c=\frac{\mathrm{R} \cos e}{v_{o}}=\mathrm{R} \frac{d \mathrm{~T}}{d \Delta}=\frac{d \mathrm{~T}}{d \theta} .
$$

Now the path is symmetrical, so that if the greatest depth for the ray is $h_{m}$, the velocity at that depth is given by $\left(\mathrm{R}-h_{m}\right) / c$. If we put $r / v=\eta$ we find that $\Delta$ and $\mathrm{T}$ are expressed as integrals, viz. :-

where

$$
\begin{aligned}
\Delta / \mathrm{R}=\theta & =2 c \int_{c}^{b}\left(\eta^{2}-c^{2}\right) \frac{-\frac{1}{2}}{d \eta} \log r d \eta \\
\mathrm{T} & =2 \int_{c}^{b}\left(\eta^{2}-c^{2}\right)^{-\frac{1}{2}} \eta^{2} \frac{d}{d \eta} \log r d \eta
\end{aligned}
$$

$$
b=\mathrm{R} / v_{0} \text {. }
$$


If the law of variation of $v$ with $r$ is known we could evaluate the integrals. We do not, however, know this law, and the problem before us is whether, from the graphical representation of $\mathrm{T}$ as a function of $\Delta$ or $\theta$ from observations, we may determine $v$ as a function of $r$.

The analytical solution is expressed by

$$
\frac{d}{d \eta} \log r=-\frac{\mathrm{I}}{\pi} \frac{\delta}{\delta \eta} \int_{\eta}^{b}\left(c^{2}-\eta^{2}\right)^{-\frac{1}{2}} \theta d c
$$

(cf. Bateman, "Phil. Mag.," I 910$)$, and

$$
\eta^{2} \frac{\delta}{\delta \eta} \log r=-\frac{\mathrm{I}}{\pi} \frac{\delta}{\delta \eta} \int_{\eta}^{b}\left(c^{2}-\eta^{2}\right)^{-\frac{1}{2}} c \mathrm{~T} d c
$$

so that if $\theta$ and $\mathrm{T}$ can be expressed as functions of $c$ or $\frac{d \mathrm{~T}}{d \theta}$ we should get $r$ as a function of $\eta$ and hence the velocity at any depth. Now the observations give $\mathrm{T}$ as a function of $\Delta$, so that theoretically the problem is solved. But as a matter of fact time curves are still very inaccurate and do not justify a very minute analysis at present.

One must proceed by a comparatively rough graphical process, and the obvious suggestion would be to take successive ranges within which $\theta$ does not vary much with $c$.

Wiechert, who first attacked the problem, divided the Earth into finite layers within each of which the radius of curvature of the path might be taken as constant, and on this basis Wiechert, Zöppritz, and Geiger (l.c.) analysed the time curves for $\mathrm{P}$ and $\mathrm{S}$. The results of the investigation which are set out in the table, page $6 \mathrm{I}$, show that from $\Delta=0$ to $\Delta=5000 \mathrm{~km} ., h_{m}$ increases from o to about $1500 \mathrm{~km}$., while $\mathrm{V}_{1}$ and $\mathrm{V}_{2}$ continually increase as $h_{m}$ increases. As $\Delta$ increases to $6000 \mathrm{~km} . h_{m}$ increases very little. Beyond this $h_{m}$ again increases until for $\Delta=\mathrm{I} 3,000 \mathrm{~km} . h_{m}$ attains a value rather over $3000 \mathrm{~km}$. But from $h_{m}=1500$ to $3000 \mathrm{~km}$. both $\mathrm{V}_{1}$ and $\mathrm{V}_{2}$ remain constant. It is specially interesting that Poisson's ratio $\sigma$ remains practically constant.

The variation of velocity with depth may not, however, be continuous, but we may have surfaces at which the velocity undergoes a sudden change. Such a surface of discontinuity 


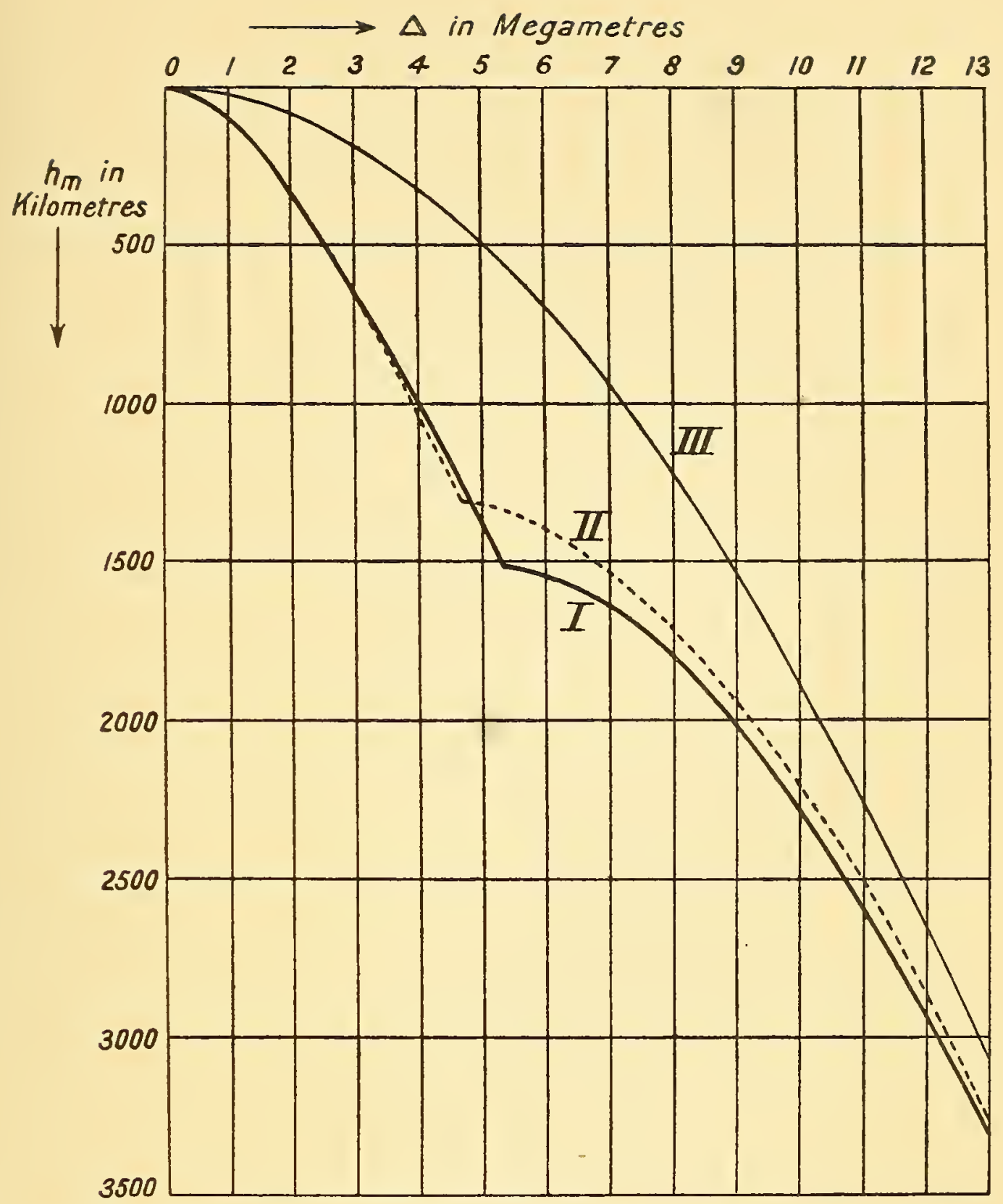

Plate 6.-Showing maximum depth of seismic rays as function of epicentral distance (after Zöppritz).

Plate 6 shows the maximum-depth $\left(h_{m}\right)$ attained as a function of the epicentral distance $\Delta$, for

I. The first phase $P$ as observed.

II. The second phase $\mathrm{S}$ as observed.

III. Theoretical straight rays with constant speed. 



\begin{tabular}{|c|c|c|c|c|}
\hline$\underset{\text { about }}{\Delta}$ & $h_{m}$ & $V_{1}$ & $V_{2}$ & $\sigma$ \\
\hline $\begin{array}{r}\mathrm{km} . \\
0 \\
\mathrm{r}, 000 \\
1,500 \\
1,800 \\
2,200 \\
2,500 \\
2,800 \\
3,200 \\
3,500 \\
3,700 \\
4,000 \\
4,300 \\
4,500 \\
4,800 \\
5,000 \\
5,300 \\
13,000\end{array}$ & $\begin{array}{r}\mathrm{km} . \\
0 \\
100 \\
200 \\
300 \\
400 \\
500 \\
600 \\
700 \\
800 \\
900 \\
\mathrm{I}, 000 \\
\mathrm{I}, 100 \\
\mathrm{r}, 200 \\
\mathrm{I}, 300 \\
\mathrm{r}, 400 \\
\mathrm{I}, 500 \\
3,300\end{array}$ & 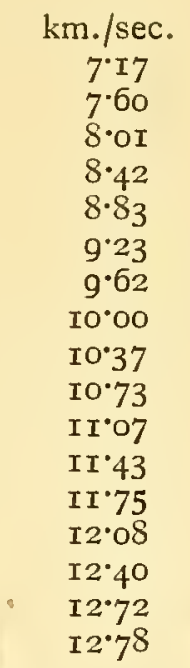 & $\begin{array}{c}\mathrm{km} . / \mathrm{sec} . \\
4^{\circ} \mathrm{OI} \\
4^{\circ} \cdot 24 \\
4^{\circ} 47 \\
4^{\cdot} 70 \\
4^{\circ} \cdot 93 \\
5^{\cdot} \cdot 15 \\
5^{\circ} 37 \\
5^{\circ} \cdot 59 \\
5 \cdot 80 \\
6 \cdot 00 \\
6 \cdot 21 \\
6 \cdot 41 \\
6 \cdot 60 \\
6 \cdot 80 \\
6 \cdot 87 \\
6 \cdot 87 \\
6 \cdot 87\end{array}$ & $\begin{array}{l}0.272 \\
0.273 \\
0.272 \\
0.272 \\
0.274 \\
0.272 \\
0.274 \\
0.273 \\
0.272 \\
0.271 \\
0.270 \\
0.270 \\
0.269 \\
0.268\end{array}$ \\
\hline
\end{tabular}

leads to singularities in the time curve. In particular Wiechert shows that if there is a sudden increase of velocity, there will be a corresponding point on the time curve at which the slope changes suddenly. It would then really consist of two portions cutting at a definite angle and there would be a certain range within which the seismograms would show two sharp impulses. If on the other hand there is a sudden reduction of the velocity there will be a gap in the time curve corresponding to a range of distance not reached by the waves.

In this way Wiechert in a recent investigation (Inter. Seis. Assoc. Manchester, I9I I) concludes that there are such surfaces of discontinuity situated at depths of 1200,1650 , and $2450 \mathrm{~km}$.; but I am not aware that any numbers have been published showing what change this makes in the table of velocities derived from his former investigation. He further concludes that for depths greater than $3000 \mathrm{~km}$. the velocities diminish gradually (see Geiger and Gutenberg, Gött. Nach., I9I 2).

Interesting as Wiechert's results are, they must be regarded as indicating the manner in which Seismology may be expected to throw light on the nature of the interior of Earth, rather than as results of great accuracy. Very slight changes in the slope of the time curve would lead to very considerable changes in the inferences; and in this respect it appears to me that we still require an analytical method which depends on the original 
time curve itself and not on the still less accurate curve expressing $\Delta$ as a function of slope $d \mathrm{~T} / d \Delta$. Different investigators give smoothed time curves which differ sufficiently to lead to very different conclusions as to the interior of the Earth. Moreover, we have seen that a smoothed curve may really involve a quite wrong method of procedure.

The primary curve itself is subject to many sources of error. Apart from actual errors of the time that do unfortunately exist at seismological stations, we have to remember that the marking of the exact instants at which $\mathrm{P}$ and $\mathrm{S}$ occur is a matter of personal judgment, and depends also on the particular instrument used and the sharpness of the impulses.

The first portion of the curve depends on the elimination of the effect of finite depth of the focus, and as that is a very difficult matter, I should doubt if it is often successfully accomplished. Again for distances much beyond $10,000 \mathrm{~km} . \mathrm{S}$ is often extremely indistinct. There are probable theoretical reasons for this as we have pointed out, but meanwhile it introduces uncertainty. Beyond $\mathrm{I} 3,000 \mathrm{~km}$. data are very meagre, and the determination of the incidence of $\mathrm{P}$ becomes increasingly difficult on account of the smallness of the horizontal movement.

Thus there is room for progress both on the theoretical and the experimental side, but the growing activity of seismologists is a good augury for the successful improvement of time curves even to the semicircumference of the Earth. 


\section{CHAPTER VIII.}

DETERMINATION OF EPICENTRE AND FOCUS.

THE first question that arises when a seismogram indicates the occurrence of an earthquake is-where did the earthquake occur?

We have hitherto regarded the earthquake as occurring at a point called the focus. Strictly the primary shock may have extended throughout a considerable region, so that in speaking of the focus we assume some average point from which the maximum effect appeared to proceed. Again we have seen that the focus may be at some depth and not at a point on the surface. For distances over $1000 \mathrm{~km}$., however, it is quite accurate enough to regard the shock as occurring at a point on the surface known as the epicentre. Several definitions of epicentre, based on different physical ideas, may be given. It may, for example, be defined as the surface point first affected by the shock, or the surface point where maximum effect is produced. For our immediate purpose it is sufficient to define the epicentre as the extremity of the Earth's radius that passes through the focus. Until quite recently the method available for obtaining the epicentre was empirical, and based on the time curves for $\mathrm{P}$ and $\mathrm{S}$ as a function of the epicentral distance $\Delta$, obtained from observations of former earthquakes with well-defined epicentres. The most accurate of these are the curves obtained by Zöppritz. We shall return to the manner in which the primary time curves are to be obtained and meanwhile suppose that the table of values of $\mathrm{S}-\mathrm{P}$ in seconds for each $10 \mathrm{~km}$. as interpolated by Zeissig is available (published by the Imp. Acad. of Sciences, St. Petersburg).

If then $\mathrm{P}$ and $\mathrm{S}$ are clearly defined on the record the interval 
$\mathrm{S}-\mathrm{P}$ is known, and the corresponding distance $\Delta$ of the epicentre from the station is determined. The result is free from any absolute error of time at the station. In many cases, however, $\mathrm{P}$ is so small that its incidence cannot be accurately assigned, and then one may get an estimate of the distance from S-PR, or L-S, but these are much less accurate and ought only to be used as a check.

When $\Delta$ is determined thus for three suitably selected stations the position of the epicentre is determined uniquely as the common point of intersection of three small circles on the sphere. Needless to say the circles do not precisely intersect at a point in practice, so that the epicentre is given only within certain limits. The co-ordinates of latitude and longitude may of course be obtained by computation or graphically on a stereographic projection.

It was pointed out by Galitzin that if the first impulse represents the arrival of a longitudinal effect in the plane containing epicentre, station, and Earth's centre, the ratio of the magnitudes of the displacements to north and to east must give the tangent of the azimuth of this plane, so that the distance and direction of the epicentre can be determined by observations at a single station. This principle has been subjected to rigorous examination first at Pulkowa and later at Eskdalemuir, and the results show quite conclusively that, provided the first impulse is sufficiently clear and large, the epicentre can be determined in this way with great accuracy. There is a possible ambiguity of $180^{\circ}$ in the azimuth determined in this way from the horizontal seismograms alone, for the first impulse may be a condensation or a rarefaction. The vertical component seismograph, however, removes the ambiguity, for if the impulse is a condensation the corresponding vertical movement is up, while for a rarefaction the vertical movement is down. There are indications that the first impulse may appear as a rarefaction at one station and as a condensation at another. This might be expected on Stokes' dynamical theory of diffraction, and if it proves correct, it suggests a means of finding the axis of the primary impulse; and this would be a valuable addition to seismological know- 
ledge. When the distance $\Delta$ and the azimuth $a$ have been determined at a station we may calculate the co-ordinates of the epicentre by means of the formulæ

$$
\sin \phi_{\mathrm{E}}=\cos \Delta \sin \phi_{\mathrm{S}}+\sin \Delta \cos \phi_{\mathrm{S}} \cos a
$$

and

$$
\cos \left(\lambda_{E}-\lambda_{S}\right)=\frac{\cos \Delta-\sin \phi \sin \phi_{E}}{\cos \phi_{S} \cos \phi_{E}}
$$

where $\phi_{S}, \lambda_{S}$ are the latitude and longitude of the station and $\phi_{E}, \lambda_{E}$ are the latitude and longitude of the epicentre.

As an illustration of the accuracy obtained by the use of Galitzin's seismographs, compare the independent determinations of the epicentre of the Monastir earthquake of I 8 February, I9I I, made at Pulkowa and Eskdalemuir.

For Pulkowa $\phi_{\mathrm{S}}=59^{\circ} 46^{\prime} \mathrm{N} \quad \lambda_{\mathrm{S}}=30^{\circ} \mathrm{I} 9^{\prime} \mathrm{E}$ and the seismogram gave $\Delta=2260 \mathrm{~km}$. $\equiv 20^{\circ} 19^{\prime}$ and $a=22^{\circ} 53^{\prime}$ West of South. Hence for the epicentre

$$
\phi_{\mathrm{E}}=40.5^{\circ} \mathrm{N} \quad \lambda_{\mathrm{E}}=20 \cdot \mathrm{I}^{\circ} \mathrm{E} \text {. }
$$

For Eskdalemuir $\phi_{\mathrm{S}}=55^{\circ} 19^{\prime} \mathrm{N} \quad \lambda_{\mathrm{S}}=3^{\circ} \mathrm{I} 2^{\prime} \mathrm{W}$ and the seismogram gave $\Delta=2360 \mathrm{~km}$. $\equiv 2 \mathrm{I}^{\circ} \mathrm{I}^{\prime}$ and $a=55^{\circ} 56^{\prime}$ East of South. Hence for the epicentre

$$
\phi_{E}=40.6^{\circ} \mathrm{N} \quad \lambda_{E}=20.3^{\circ} \mathrm{E} .
$$

As long as the first impulse is really sharp no trouble arises; but with a small and gradual start, it is sometimes difficult to identify the corresponding movements on the horizontal and vertical seismograms, owing to a phase difference of the maximum displacement. Thus instruments with the same fundamental constants are required to remove this source of error of judgment.

It is clear that if the azimuths have been accurately determined at two stations the epicentre can be determined from these alone without reference to the determinations of distance (see Galitzin and IWalker, "Nature," August, 1912).

The preceding example gives in this way

$$
\phi_{\mathrm{E}}=40^{\circ} 4^{\circ} \mathrm{N} \quad \lambda_{\mathrm{E}}=20^{\circ} 3^{\circ} \mathrm{E} \text {. }
$$

for the epicentre, while the deduced distances from Pulkowa and Eskdalemuir are then $20^{\circ} \mathrm{I} 8^{\prime}$ and $21^{\circ} 26^{\prime}$ respectively. 
The three values for the epicentre do not differ by more than $20 \mathrm{~km}$.

The advantages of this method are that it is quite independent of (I) the time at the two stations, and (2) the determination of $S$, and thus free from any error that attaches to the empirical time curves. It should thus prove of great value in improving the empirical time curves, more especially for short distances where the influence of finite depth of focus is considerable. For this reason I consider that an instrument which would give the azimuth directly would be of great service even if the remaining part of the seismogram had to be sacrificed.

We have now to consider how the primary time curves are to be obtained.

We shall suppose that we have available the times of incidence of $\mathrm{P}$ and $\mathrm{S}$ at a number of stations. Before these can be arranged we require to know the position of the epicentre so that the distances $\Delta$ can be computed. In some cases (e.g. the great Messina earthquake, I908) the epicentre is known with considerable accuracy from local knowledge. But, in many cases such information is not available or cannot be relied on, and then some other method must be used.

We have seen that an extension of Galitzin's method of azimuths may give the epicentre directly. So far it has not been used in the preparation of time curves, but there is little doubt that it is the most satisfactory method we can have.

When observations of $\mathrm{P}$ have been obtained at several stations known to be not very far from the epicentre, we may however get a fairly good determination of the position of the epicentre by a method used by Zöppritz (Gött. Nach., I907, 1.c.). If for instance $P$ occurs at precisely the same instant at three stations not too far from the epicentre, the epicentre would be the unique point which is equidistant from the three stations. If the times differ we may proceed as follows: Let $A, B$, and $C$ be the stations and let $X$ be the epicentre; we then have the equations

$$
\mathrm{XA}=v_{11}(y), \quad \mathrm{XB}=v_{0}(y+p), \quad \mathrm{XC}=v_{0}(y+q)
$$

where $p$ and $q$ are the observed time intervals in seconds 
between $\mathrm{B}$ and $\mathrm{A}$, and $\mathrm{C}$ and $\mathrm{A}, v_{0}$ the velocity of propagation of the disturbance, and $y$ the unknown time from epicentre to A. We may then by trial construct the circles of radii proportional to $y, y+p, y+q$ with centres at $\mathrm{A}, \mathrm{B}, \mathrm{C}$ which intersect in a point, and we then get the position of the epicentre and also the time $y$ from $\mathrm{A}$ to $\mathrm{X}$. The above equations are approximate and do not take account of the depth of focus. But is we shall show in a little if the distances are within from 200 to $400 \mathrm{~km}$., the error introduced in the times is less than half a second even for a focus $40 \mathrm{~km}$. deep, and the observed times are not accurate to this extent. The time $y$ is then the time from $\mathrm{A}$ to the focus or to the epicentre, to less than half a second, but we must be careful to observe that the time from focus to epicentre is not zero. For the formulæ become inaccurate beyond the range given.

Having obtained the epicentre we may now set out the curves giving $\mathrm{P}$ and $\mathrm{S}$ as a function of the distance $\Delta$, and if we accept the time of occurrence at the focus given by deducting the time $y$ from the time at $\mathrm{A}$, we complete our time curve giving the interval of time from focus to station as a function of the arc from epicentre to station. We may not, however, exterpolate the curve to points quite close to the epicentre, until we know the depth of the focus.

The curves we have obtained are still time curves depending on the depth of focus. There is a range of several hundred kilometres within which the influence of depth is extremely small, but for shorter distances the influence of depth is considerable and again for greater distances the error may amount to a few seconds.

The curve cannot be freed from the effect of depth and so prepared for theoretical investigation unless we know the depth of focus or have observations sufficiently near the epicentre to determine it. Zöppritz (I.c.) proposed the following method of correcting the time curves when the depth $h$ has been obtained. Assuming that the path (fig. I 3 ) is symmetrical we may prolong the path SF backwards to meet the earth's surface at $\mathrm{O}$, and the angle $\mathrm{EOF}=e$ is equal to the angle of emergence at the station. Thus $\mathrm{OE}=\mathrm{EF} \cot e=h \cot e$ and the time to 
traverse OF would be $\mathrm{OF} / v_{0}=h \operatorname{cosec} e / v_{0}$ where $h$ is the depth of the focus and $v_{0}$ the velocity of the disturbance at the surface. Thus for great distances we may pass to the corrected

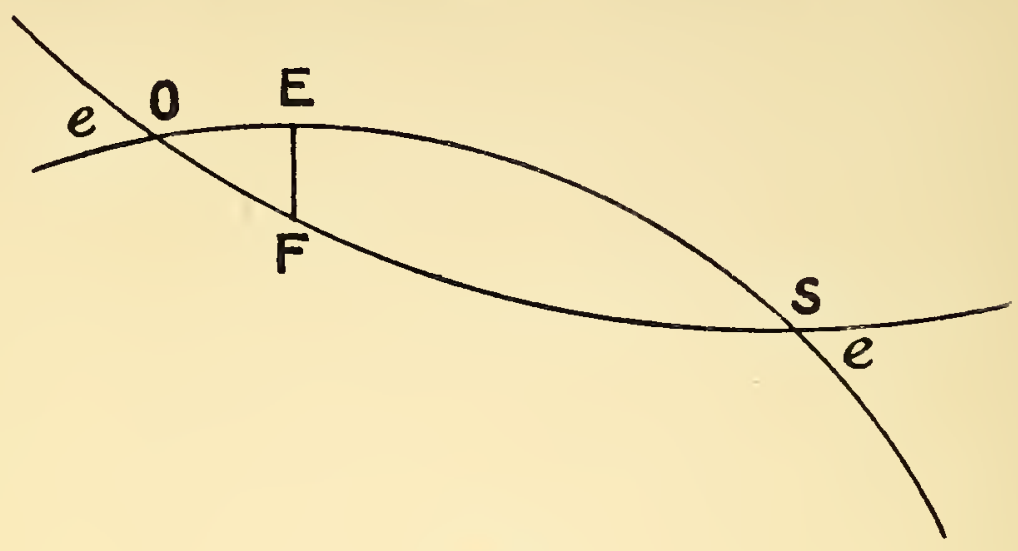

FIG. I3.

curve by applying to the original point $(t, \Delta)$, the corrections $\delta$, and $\delta \Delta$ where $\delta t=h \operatorname{cosec} e / v_{0}, \delta \Delta=h \cot e$. The corrections would, of course, differ for the $\mathrm{P}$ and $\mathrm{S}$ curves and $e$ would be determined from the corresponding curve.

This procedure is probably accurate enough for distances $>1,000 \mathrm{~km}$., but entirely breaks down as we get close to the epicentre. In any case no correction can be attempted until $h$ is known. Thus we may now consider how, if at all, $h$ can be obtained by observation.

It seems evident that only observations not far from the epicentre would be of much use for this purpose, but what I think one is hardly prepared for is the extreme closeness to the epicentre required, if we are to depend on the times of arrival of $\mathrm{P}$ for the determination of $h$.

It is not often that data are available which make any attempt to determine the depth of focus worth while, but the occurrence of an earthquake in South Germany on 16 November, I9I I, tempted several investigators to see what could be made out as to the depth. Galitzin (Nach. d. Seis. Comm. Petersburg, $\mathrm{Bd}$. V. L3, I9I2) went into the problem very carefully, but it is to be feared that the data finally proved to be too unsatisfactory to justify an elaborate analysis.

Galitzin first attempts to take account of the influence of depth on the velocity of propagation of the longitudinal 
disturbance. He assumes as an approximate law for small depths

$$
\nu^{2}=\left(\frac{v_{0}}{v}\right)^{2}=I+c(x-I)
$$

where

$$
x=\left(\mathrm{I}-\frac{h}{\mathrm{R}}\right)^{2},
$$

and $v_{0}$ is the velocity at the surface, $v$ the velocity at depth $h$, and $\mathrm{R}$ the earth's radius. Now Zöppritz' results give

$$
\begin{aligned}
& v_{0}=7.17 \mathrm{~km} . / \mathrm{sec} \\
& v_{100}=7.60 \mathrm{~km} . / \mathrm{sec}
\end{aligned}
$$

and hence $c=3.529$ while $\mathrm{R}=6370 \mathrm{~km}$.

\begin{tabular}{|c|c|c|c|c|c|}
\hline \multirow{2}{*}{$\begin{array}{c}\text { Distance } \\
\Delta \mathrm{km} .\end{array}$} & \multicolumn{3}{|c|}{ Time from focus to station in secs. } & \multicolumn{2}{|c|}{ Differences. } \\
\hline & $h=\mathrm{I} \mathrm{km}$. & $h=10 \mathrm{~km}$ & $h=40 \mathrm{~km}$ & $\mathrm{~T}_{10}-\mathrm{T}_{1}$ & $T_{10}-T_{1}$. \\
\hline & S. & s. & s. & S. & S. \\
\hline 0 & $0 \cdot 13$ & $I^{\circ} 43$ & $5 \cdot 52$ & $+1 \cdot 30$ & +539 \\
\hline 50 & $7 \cdot 35$ & $6 \cdot 99$ & $8 \cdot 80$ & $-0 \cdot 34$ & $+I^{\circ} 47$ \\
\hline IOO & $13 \cdot 83$ & I3.96 & $I_{4} \cdot 80$ & $+0 \cdot I_{3}$ & $+0 \cdot 97$ \\
\hline 150 & $20 \cdot 80$ & $2 \mathrm{I} \cdot \mathrm{I} 2$ & $2 I \cdot 32$ & +0.32 & +0.52 \\
\hline 200 & $27 \cdot 67$ & $27^{\circ} 57$ & $28^{\circ} 00$ & - OPIO & +0.33 \\
\hline 250 & $34^{\cdot 80}$ & $34^{\circ} 65$ & $34^{\circ} 7^{6}$ & $-0 \cdot 15$ & -0.04 \\
\hline 300 & $4 I \cdot 74$ & $4 I \cdot 56$ & $4 I \cdot 5 I$ & $-0 \cdot 18$ & -0.23 \\
\hline $35^{\circ}$ & $4^{8 \cdot 7 I}$ & $48 \cdot 58$ & $48 \cdot 42$ & $-0 . I_{3}$ & -0.29 \\
\hline 400 & $55^{\circ} 25$ & $55^{\circ} 44$ & $55^{\circ} 05$ & + O.Ig & -0.20 \\
\hline $45^{\circ}$ & $62 \cdot 44$ & $62 \cdot 29$ & $6 I \cdot 80$ & - $0^{*} \mathrm{I}_{5}$ & $-0 \cdot 64$ \\
\hline 500 & $69 \cdot 43$ & $69^{\circ} I_{4}$ & $68 \cdot 55$ & -0.29 & -0.88 \\
\hline
\end{tabular}

Integral expressions for the distance $\Delta$ and the time $\mathrm{T}$ from focus to station are then obtained and used to compute the following among other tables.

The columns of differences suggest that some error of computation has crept into the numbers.

The table on the following page is obtained on the simple hypothesis that the velocity is constant for any depth here considered and equal to $7 \cdot 17 \mathrm{~km}$. per second.

Several points are suggested by a comparison of these tables. We notice that the point of inflexion on the time curve is so ill defined that it is useless for estimating $h$. Further, anywhere between 200 and $400 \mathrm{~km}$. is quite useless to attempt to discriminate between the two tables or for any value of $h$ 
up to $40 \mathrm{~km}$. by means of observations which are only given to the nearest second. Only at $500 \mathrm{~km}$. and then only for $40 \mathrm{~km}$. depth do the two values differ by I second, and as a matter of fact we can hardly suppose the value of $v_{0}$ to be so accurately known as to give much security.

\begin{tabular}{|c|c|c|c|c|c|}
\hline \multirow{2}{*}{$\begin{array}{c}\text { Distance } \\
\Delta .\end{array}$} & \multicolumn{3}{|c|}{ Time from focus to station in secs. } & \multicolumn{2}{|c|}{ Differences. } \\
\hline & $h=\mathrm{I} \mathrm{km}$ & $h=10 \mathrm{~km}$. & $h=40 \mathrm{~km}$. & $\mathrm{T}_{10}-\mathrm{T}_{1}$. & $T_{40}-T_{1}$. \\
\hline & s. & s. & s. & & \\
\hline 0 & O. I4 & I.39 & $5^{\circ} 5^{8}$ & $I \cdot 25$ & $5^{\circ} 44$ \\
\hline 50 & $6 \cdot 98$ & $7^{\circ} \mathrm{II}$ & $8 \cdot 92$ & 0.13 & I. 94 \\
\hline IOO & I3.95 & $\mathrm{I}_{4} \cdot \mathrm{OI}$ & $I_{4} \cdot 99$ & $0 \cdot 06$ & $\mathrm{I} \cdot \mathrm{O}_{4}$ \\
\hline I5O & $20^{\circ} 9 \mathrm{I}$ & $20 \cdot 94$ & $2 \mathrm{I} \cdot 57$ & 0.03 & 0.66 \\
\hline 200 & $27 \cdot 88$ & $27^{\circ} 90$ & $28 \cdot 35$ & 0.02 & 0.47 \\
\hline 250 & $34 \cdot 8 \mathrm{I}$ & $34 \cdot 8 \mathrm{I}$ & $35 \cdot 14$ & 0.00 & 0.33 \\
\hline 300 & $4 I \cdot 83$ & $4 \mathrm{I} \cdot 8_{3}$ & $42 \cdot 08$ & 0.00 & 0.25 \\
\hline 350 & $48 \cdot 8 \mathrm{I}$ & $48 \cdot 79$ & $4^{8 \cdot 98}$ & -0.02 & 0.17 \\
\hline 400 & $55^{\circ} 76$ & $55^{\circ} 73$ & $55 \cdot 86$ & -0.03 & $0 \cdot 10$ \\
\hline 450 & $62 \cdot 78$ & $62 \cdot 75$ & $62 \cdot 84$ & -0.03 & 0.06 \\
\hline 500 & $69 \cdot 75$ & $69 * 72$ & $69 \cdot 76$ & -0.03 & O॰OI \\
\hline
\end{tabular}

We may, however, conclude that on either hypothesis the observations between 200 and $400 \mathrm{~km}$. should give us the actual time of occurrence of the shock at the focus to less than $\frac{1}{2}$ second as practically independent of $h$ for $h<40 \mathrm{~km}$. and that is an important point gained. Next, to get the depth we must use only the observations for $\Delta<200 \mathrm{~km}$. and even then it is really only the observations for $\Delta<50 \mathrm{~km}$. that ought to count heavily. Here also it is impossible to discriminate practically between the two hypotheses, so that the simpler one should have the preference.

Turning now to the actual data in the table on the opposite page, we note that the distances were computed from the epicentre determined by noting that the times at Zürich and Strassburg were the same, as were also the times at Aachen and Göttingen. The co-ordinates so obtained were

$$
\phi_{0}=48^{\circ} \mathrm{I} 9^{\prime} \mathrm{N} \text { and } \lambda_{0}=9^{\circ} 23^{\prime} \mathrm{E} \text {. }
$$

Galitzin, from the time at Karlsruhe, Strassburg, and Zürich, finds the time at the focus to be 2 I hours, 25 minutes, 52.5 seconds, and his conclusion is that the depth was $9.5 \mathrm{~km}$. with a probable error $\pm 3.8 \mathrm{~km}$. The data, however, show dis- 
crepancies of as much as 2 seconds. These may be quite real, for it is not unlikely that the velocity may differ sufficiently in different directions to account for this.

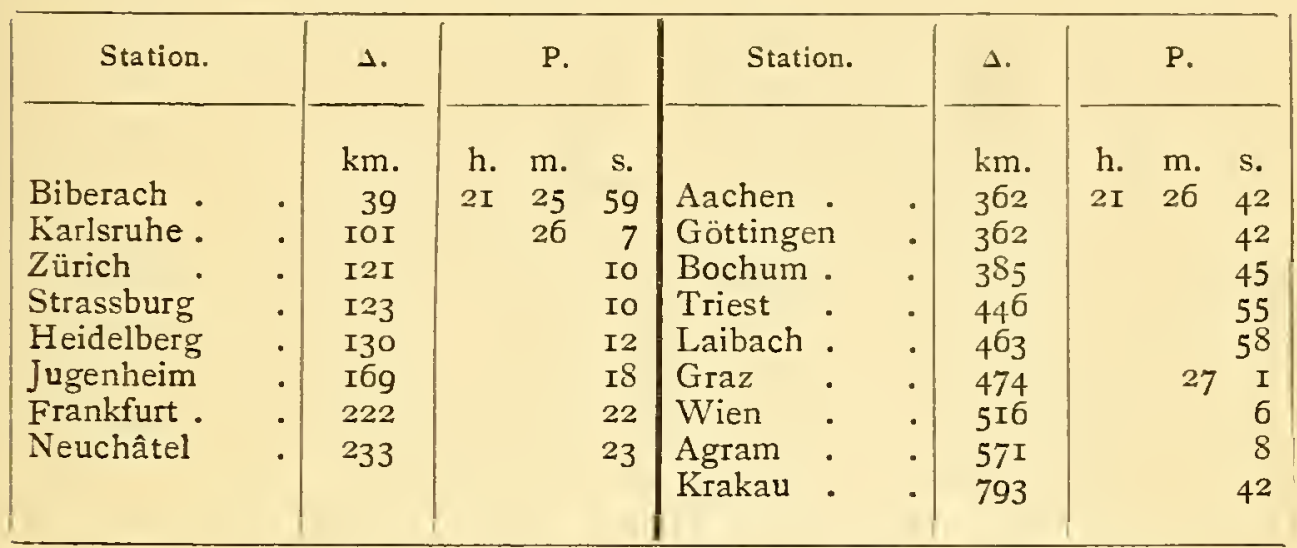

From the simpler theoretical table we get the following times to the nearest second.

\begin{tabular}{|c|c|c|c|c|c|c|}
\hline & \multicolumn{3}{|c|}{ T. } & \multicolumn{3}{|c|}{$T_{0}$ from the data. } \\
\hline & $h=\mathrm{I} . \mathrm{km}$. & $h=\mathrm{lo} . \mathrm{km}$ & $h=40 . \mathrm{km}$. & $h=\mathrm{I} . \mathrm{km}$ & $h=$ Io. $\mathrm{km}$. & $h=40 . \mathrm{km}$. \\
\hline $\mathrm{km}$. & s. & s. & s. & h. m. s. & h. m. s. & h. m. s. \\
\hline $\begin{array}{r}39 \\
\text { IOT }\end{array}$ & $\begin{array}{r}6 \\
I_{4}\end{array}$ & $\begin{array}{r}6 \\
14\end{array}$ & $\begin{array}{r}8 \\
I 5\end{array}$ & $\begin{array}{lll}2 & 25 & 53 \\
53\end{array}$ & 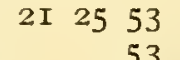 & $\begin{array}{lll}2 I & 25 & 5 \mathrm{I} \\
& & \end{array}$ \\
\hline I 22 & $\begin{array}{l}4 \\
17\end{array}$ & $\begin{array}{l}14 \\
17\end{array}$ & I8 & $\begin{array}{l}53 \\
53\end{array}$ & $\begin{array}{l}53 \\
53\end{array}$ & $\begin{array}{l}52 \\
52\end{array}$ \\
\hline 130 & I8 & I3 & Ig & 54 & 54 & 53 \\
\hline وكَ & 24 & 24 & $24^{\circ} 5$ & 54 & 54 & 53.5 \\
\hline
\end{tabular}

The conclusion is that $h$ was not as great as $40 \mathrm{~km}$. and that $10 \mathrm{~km}$. is better, but on the data we can hardly say that $h$ might not have been zero.

What seems to be clear is that unless the times were known to $O^{\cdot} \mathrm{I}$ second, only observations at less than $50 \mathrm{~km}$ would be of value to settle the matter. From a human point of view one hopes that no such case will ever occur, and the problem of finding the depth of the focus is more likely to be solved by direct observation of the emergence angle with horizontal and vertical seismographs combined. 


\section{CHAPTER IX.}

SEISMIC EFFECTS OTHER THAN THOSE DUE TO EARTHQUAKES.

DR. MiLne once remarked to me that a seismogram always has something to show worth knowing even if there is no earthquake. Those who have had the great privilege of visiting the observatory at Shide and seeing Dr. Milne's wonderful album of seismograms will appreciate how true the remark is, and how thoroughly Milne has devoted himself to anything that can throw light on the subject which he has so conspicuously adorned.

We must pass over the spurious effects on a seismograph produced by the presence of the observer, the shutting of doors, and that bane of the experimentalist, the ubiquitous spider. They are mentioned here, only to point out that the practical seismologist must be able to recognize such effects when they occur.

It was long ago recognized by Milne that a seismograph frequently shows minute vibrations continuing for many hours. and that they could not be accounted for by earthquakes or local traffic.

These effects were called by him "Tremors" and although they occur always with high local winds, they also appear when it is quite calm.

On the Milne seismograms the tremors present the same appearance on calm or on gusty days. But with heavily damped seismographs, using larger magnification and higher speed of registration, it is found that there are two types of tremors or microseisms as they are now called. They are shown to special advantage on Galitzin seismograms.

In the first class, which occurs on windy and calm days alike, the movements are very smooth and regular, and the 



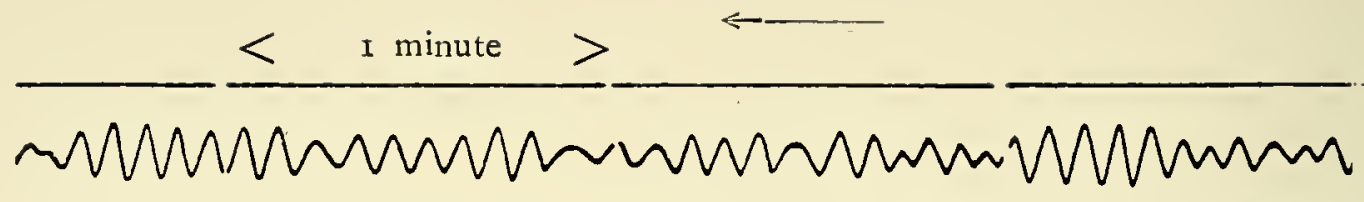

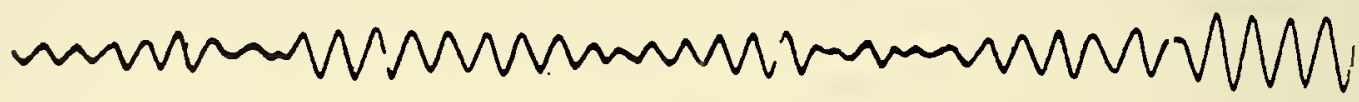

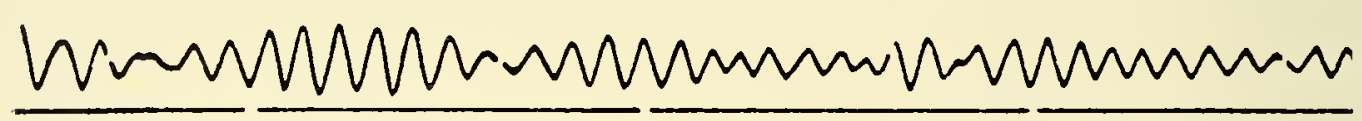

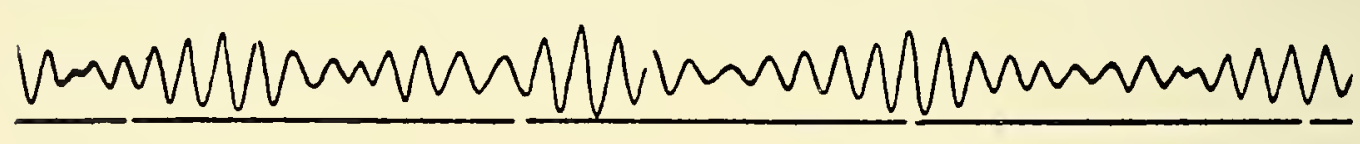

WWWWm-Mnmms

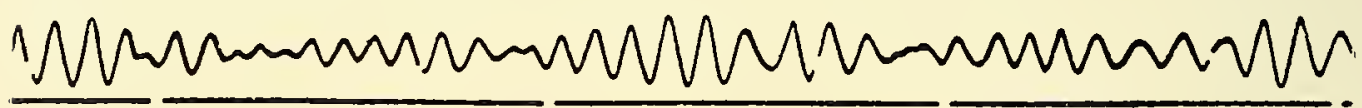

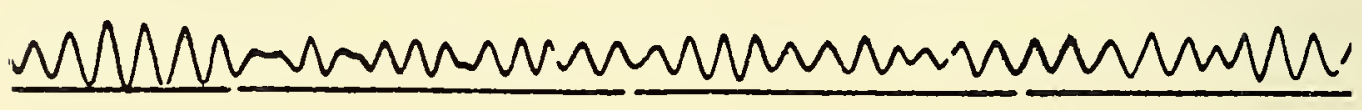

MMMmmmanmm

Plate 7A.-Portion of Record at Pulkowa, September I8, I9ro, showing microseismic movement

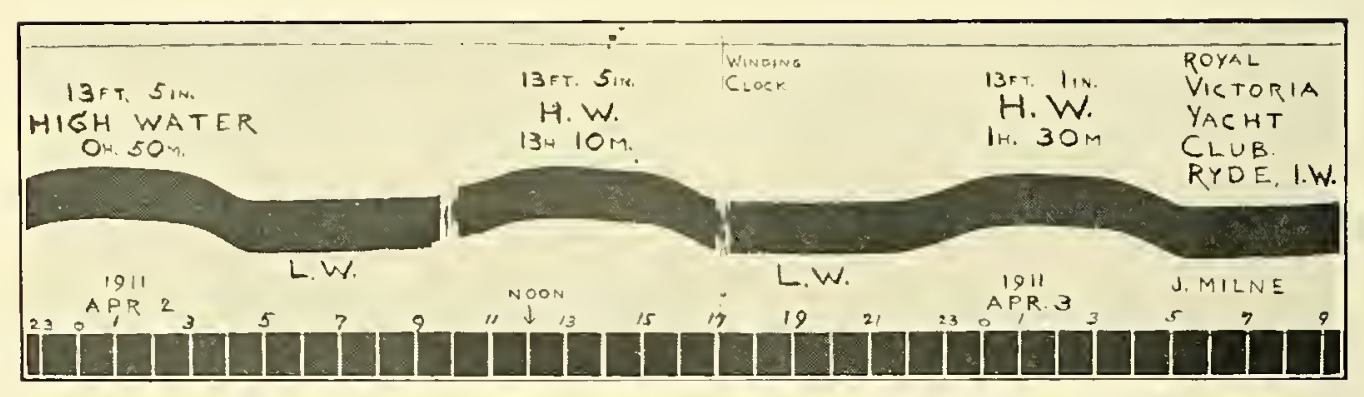

Plate 7B.-Specimen Record (reduced) showing Tilt produced by Tidal loading.

Original scale $\mathrm{I} \mathrm{mm}=\mathrm{O}^{\prime \prime \prime} \mathrm{I} 7 \mathrm{~T}$ ilt, and $\mathrm{to} \mathrm{ft}$. Tide gives $5 \mathrm{~mm}$. deflexion. 
periods range from about 4 seconds to over 8 seconds. The periods are not mixed up, for the same period will persist for many hours. In the second class the periods range from about $\mathrm{I} 2$ seconds to about 30 seconds. The movements are irregular, look like badly drawn sinusoidal curves, and the periods occur indiscriminately. These occur only on windy days.

There seems to be little doubt that the second class is due to the gusts of local wind setting the ground and buildings into movement, for they start with the wind and cease as soon as the wind subsides. In my own experience the movements are not very pronounced until the speed of the wind is about 20 miles per hour, and I should say that the movements tend to become more regular and of shorter period as the wind increases in speed. It has been found at Pulkowa that the amplitude is much reduced in an underground room, and that it is an advantage to prevent direct access of air to the sides of the piers.

Microseisms of the first type present an interesting problem for solution. They are observed at quite inland stations and at considerable depths as well as at stations near the coast. A systematic comparison of observations has been undertaken by the International Association of Seismology, but results are not yet available. The main features, are, however, fairly definite. The longer periods are associated generally with larger amplitude. The longer period movements ( 8 seconds) come out strongly in stormy weather, but persist for many hours after all local wind has ceased, and then the period and amplitude usually gradually diminish until a normal period of from 4 to 5 seconds prevails it may be for several days. Again the microseismic movement of this type is more pronounced in winter than in summer. Indeed there are often occasions in summer where the movement becomes imperceptible and this is rarely the case in winter.

Plate $7 \mathrm{~A}$ is a reproduction of a portion of a specimen record obtained at Pulkowa. It shows clearly a feature usually to be observed, that the amplitude rises to a maximum and then subsides, the maxima being at intervals of about $\mathrm{I}$ minute. 
The following table gives the average amplitude and period observed at Eskdalemuir on the Galitzin horizontal seismograms.

\begin{tabular}{|c|c|c|c|c|c|}
\hline $\begin{array}{c}\text { I9II } \\
\text { Month. }\end{array}$ & $\begin{array}{l}\text { Period } \\
\text { seconds. }\end{array}$ & $\begin{array}{l}\text { Amplitude of } \\
\text { Earth Movement } \\
\text { in Microns } \mu \text {. }\end{array}$ & $\begin{array}{l}\text { IgII } \\
\text { Month. }\end{array}$ & $\begin{array}{l}\text { Period } \\
\text { seconds. }\end{array}$ & $\begin{array}{l}\text { Amplitude of } \\
\text { Earth Movement } \\
\text { in Microns } \mu \text {. }\end{array}$ \\
\hline Jan. & $6 \cdot 6$ & $2 \cdot 2$ & July & $4 \cdot 3$ & 0.3 \\
\hline Feb. & $6 \cdot 0$ & $2 \cdot I$ & Aug. & $4^{.2}$ & 0.3 \\
\hline Mar. & $5 \cdot 5$ & $I \cdot 2$ & Sept. & 5.5 & 0.6 \\
\hline Apr. & $5 \cdot 5$ & 0.7 & Oct. & $4^{\circ} 9$ & 0.5 \\
\hline May & $5 \cdot 2$ & 0.6 & Nov. & 5.4 & $I \cdot 8$ \\
\hline June & 4.5 & 0.4 & Dec. & $5 \cdot 3$ & $\mathrm{I} \cdot 8$ \\
\hline
\end{tabular}

Average for year : Period $=5^{\cdot 2}$ seconds; Amp. $=\mathrm{I} \cdot 0 \mu$.

The vertical movement is quite as pronounced as the horizontal movement, and this suggests that we are dealing with Rayleigh waves propagated over large continental areas.

The general phenomena and the periods presented by these microseismic movements correspond so closely with what one observes of the sea waves on the coast, that one can hardly doubt that the two things are closely connected. Dr. Schuster has devised and set up an apparatus near Newcastle for obtaining a continuous register of the sea waves, but detailed results are not yet available for comparison with the movements shown by seismographs.

It has been suggested that the land effects are due to the actual breaking of waves on the coast, but this can hardly be maintained as an explanation of effects observed so far inland as central Europe or central Canada. It seems more probable that, and it is at least worth while investigating theoretically whether, the motion observed far inland is due to Rayleigh waves set up at the bottom of the sea by water waves set up and maintained over large ocean areas by the wind. To take a simple example: we know that a travelling wind sets up a train of waves following after it. On deep water, such as midAtlantic, we should get a period of 5 seconds, wave length $40 \mathrm{~m}$., with a wind velocity of $8 \mathrm{~m}$. per second, or about 20 miles per hour; while a period of Io seconds, wave length I $60 \mathrm{~m}$., requires a wind velocity of $16 \mathrm{~m}$. per second. Such waves advancing 
into shallower water would maintain their period but diminish in wave length and speed; while the amplitude of movement at a depth equal to the wave length would be I/500 of the surface amplitude. This would seem to provide adequate margin for explaining an observed earth amplitude of $\mathrm{I}$ micron $=$ oO I $\mathrm{mm}$. even at a considerable distance from the area of origin.

The case of waves set up by wind in an ocean of moderate depth, such as the North Sea (average depth about $100 \mathrm{~m}$.) is more complex, but is soluble on the lines indicated by Lamb ("Hydrodynamics") and seems to merit investigation with a view to explaining the microseismic movement observed in Western Europe.

Dr. Klotz of Ottawa, who has studied the effects observed there by means of a Bosch Seismograph, is of opinion that the largest effects are associated with cyclonic areas in the North Atlantic, and he suggests that the microseismic movement may appear in West Europe before the cyclone arrives. If this should prove to be the case it would be a most valuable addition to meteorological knowledge.

In Chapter I. we have observed that a pendulum, whether of simple or of horizontal type, indicates by its relative motion not only horizontal acceleration applied to the pier, but also tilting. It also indicates accelerating effect applied to the mass in a horizontal direction. If these effects are applied very slowly, the inertia and frictional terms in the equation of motion have no influence and the pendulum simply shows a gradual change of its zero position. The equation is now of the form

$$
n^{2} \theta=(\ddot{x}-g \psi) / / \text { or } i \theta=\frac{\mathrm{I}}{g}(\ddot{x}-g \psi)
$$

wherein $\theta$ and $\ddot{x}$ are measured to the right and $\psi$ is measured in the anti-clockwise direction.

The changes of zero are shown by all mechanical pendulums, but it must be remembered that here the electromagnetic method of registration is of no avail, since the zero position of the galvanometer is not dependent on the zero position of the pendulum itself. 
It is perhaps needless to remark that the zero of a pendulum is continually changing. Such changes may be merely instrumental or due to local temperature change. As such they are of little scientific interest, and are rather a serious nuisance, and every care should be taken to remove such sources of change. Careful examination shows, however, that part of the change of zero is regular and of considerable scientific importance. The most marked effect in point of magnitude is that which occurs on the seismograms of the Milne pendulum, e.g. at Ryde, Isle of Wight, which show in a manner visible to the eye a regular sinusoidal movement of the zero agreeing precisely with the rise and fall of the Channel tides. There seems little doubt that the rock strata bend under the influence of this periodic alteration of load in the Channel basin. Dr. Milne has kindly sent me the specimen record, Plate $7 \mathrm{~B}$.

Such visible effects are not however shown at inland stations, and it is only by careful analysis of results extending over long intervals that the existence of periodic movement in the pendulum zero can be detected. The effects, although small, derive importance from their association with earth tides and the theory of the physical properties of the Earth.

The acceleration of gravity $g$ at any point of the Earth's surface is not exactly constant either in magnitude or direction, but on account of the attraction of the Sun or Moon it undergoes small changes. The potential of these additional forces at any point is expressed by a solid spherical harmonic of order 2 and may be written

$$
W_{2}=\frac{3}{2} m g \frac{a^{2} r^{2}}{c^{3}}\left(\cos ^{2} \theta^{\prime}-\frac{1}{3}\right)
$$

wherein $m$ is the mass of the Sun or Moon,

$c$ is the distance of the Sun (or Moon) from the

Earth's centre,

$g$ the normal acceleration of gravity,

$a$ the mean radius of the Earth,

$r$ the geocentric radius to the point, and

$\theta^{\prime}$ the geocentric zenith distance of the Sun or Moon.

The solar effect is about half that of the Moon.

If now $x$ is any direction on the Earth's surface perpendi- 
cular to the original direction of $g$, the potential $\mathrm{W}_{2}$ will give rise to an accelerating force

$$
\ddot{x}=\frac{\partial W_{2}}{\partial x}
$$

which is operative in deflecting the pendulum. But this is not the whole matter. The Earth yields to the disturbing potential $W_{2}$ and, in accordance with a well-established principle, the surface is deformed by an earth tide of amount $h \mathrm{~W}_{2} / g$ and the deformation of both earth and sea produces an extra potential $k \mathrm{~W}_{2}$. Thus the additional force operative on the pendulum mass becomes

$$
(I+k) \frac{\partial W_{2}}{\partial x}
$$

instead of

$$
\frac{\partial W_{2}}{\partial x} \text {. }
$$

The tide $h W_{2} / g$, however, produces a tilt in the platform

$$
\psi=\frac{h}{g} \frac{\partial W_{2}}{\partial x}
$$

so that the recorded displacement of the pendulum zero will appear to be proportional to

instead of

$$
(\mathrm{I}-h+k) \frac{\partial \mathrm{W}_{2}}{\partial x}
$$

$$
\frac{\partial W_{2}}{\partial x}
$$

as it would be if the Earth did not yield.

In a similar way it appears that the oceanic tide becomes $(\mathrm{I}-h+k) \mathrm{W}_{2} / g$ in place of $\mathrm{W}_{2} / g$.

When the potential $W_{2}$ is expressed in terms of the latitude and longitude and the co-ordinates of Sun or Moon, we obtain a number of terms representing the component tides. Chief among these are the approximately semi-diurnal lunar and solar terms, and for reasons that do not appear quite adequate, attention has until recently been concentrated on the corresponding terms in the pendulum zero movement.

The experiments begun by Zöllner and Rebeur-Paschwitz have been repeated by others, and the most recent observations 
are those by Hecker, Orloff, and Schweydar. Hecker's results are to be found in "Publications of the Royal Prussian Geodetic Institute," No. 32, I907, and No. 49, I9I I.

His observations were made at Potsdam with RebeurPaschwitz pendulums at a depth of $25 \mathrm{~m}$. so as to secure constant temperature.

The azimuth of Pendulum I was $42^{\circ} \mathrm{E}$ of $\mathrm{N}$, and of Pendulum II $48^{\circ} \mathrm{W}$ of $\mathrm{N}$.

The semi-diurnal disturbing potential may be written

$$
\mathrm{M}_{2}=\frac{3}{4} \frac{a^{2} r^{2}}{c^{3}} m g\left(\mathrm{I}-\frac{5}{2} e^{2}\right) \cos ^{2} \frac{w}{2} \cos ^{2} \phi \cos 2\left(t+\lambda-t_{0}\right)
$$

where $\phi$ and $\lambda$ are the latitude and longitude, $e$ and $w$ the eccentricity and inclination of the orbit, and $t$ is the lunar or solar time, as the case may be, referred to some convenient origin.

It is usual to express the observations not in terms of the force

$$
\frac{\partial M_{2}}{\partial x}
$$

but in terms of the apparent angular change of the direction of gravity, viz. :-

$$
\frac{\mathrm{I}}{g} \frac{\partial \mathrm{M}_{2}}{\partial x}
$$

Hecker's latest results are as follows :-

Solar Effect.

Pendulum I.

Pendulum II.

$\begin{array}{ccc}\text { Theoretical } \frac{\mathrm{I}}{g} \frac{\partial \mathrm{M}_{2}}{\partial x} . & \mathrm{o}^{\prime \prime} \cdot 00399 \cos \left(2 t-305^{\circ} 5^{\circ}\right) & \mathrm{o}^{\prime \prime} \cdot 00389 \cos \left(2 t-48 \cdot 7^{\top}\right) \\ \text { Observed. } & \mathrm{o}^{\prime \prime} \cdot 00353 \cos \left(2 t-255^{\circ} 8^{\circ}\right) & \mathrm{o}^{\prime \prime} \cdot 00448 \cos \left(2 t-36 \cdot 6^{\circ}\right)\end{array}$

Lunar Effect.

Pendulum I.

Pendulum II.

Theoretical $\frac{\mathrm{I}}{\mathrm{g}} \frac{\partial \mathrm{M}_{2}}{\partial x} \cdot \quad \mathrm{o}^{\prime \prime} \cdot 00922 \cos \left(2 t-305^{\circ} 5^{\circ}\right) \quad \mathrm{o}^{\prime \prime} \cdot 00900 \cos \left(2 t-4^{\circ} \cdot 7^{\circ}\right)$

Observed. $\mathrm{o}^{\prime \prime} \cdot 00560 \cos \left(2 t-293^{\circ} 6^{\circ}\right) \quad \mathrm{o}^{\prime \prime} \cdot 00490 \cos \left(2 t-59^{\circ} 7^{\circ}\right)$

If the lunar effects are computed for the geographical directions we get

Lunar Effect.

N.-S.

E. -W.

Theoretical.

$0^{\prime \prime} \cdot 00788 \cos \left(2 t-180^{\circ}\right) \quad 0^{\prime \prime} \cdot 00999 \cos \left(2 t-270^{\circ}\right)$

Observed.

$\mathrm{O}^{\prime \prime} \cdot 00355 \cos \left(2 t-175^{\circ}\right) \quad \mathrm{O}^{\prime \prime \prime} 00665 \cos \left(2 t-270^{\circ} 2^{\circ}\right)$ 
It is evident at 'once that these results are not concordant either in phase or amplitude, as each term gives a different value for what is presumably the ratio' $(\mathrm{I}-h+k)$. Before proceeding farther we may remark that the general sensitiveness of the record was about $\mathrm{I} \mathrm{mm}$. $\equiv \mathrm{O}^{\prime \prime} \cdot \mathrm{O} 4$ and that much larger zero movements occurred than those expressed by the above terms. Thus it is open to doubt whether these discrepancies have any real significance, and whether the apparatus is really capable of giving more than the general order of magnitude of the effect.

The ratio for $(\mathrm{I}-h+k)$ given by Hecker's results are for the lunar terms.

0.68 for the $\mathrm{E}-\mathrm{W}$ component.

0.43 for the $\mathrm{N}-\mathrm{S}$ component.

Orloff ("Veröff d. Dorpater Sternwarte," I 9 I r) observing at Dorpat with Zöllner pendulums in the geographical directions obtained

0.68 for the E-W component.

0.59 for the $\mathrm{N}-\mathrm{S}$ component.

His apparatus was about four times as sensitive as Hecker's and the individual results show better concordance than those of Hecker.

It may be said that observers on the whole have obtained something like $2 / 3$ for the value of $(\mathrm{I}-h+k)$ from pendulum observations of this particular lunar term, and this is also the value obtained by Darwin from his analysis of the fortnightly oceanic tides in the Indian Ocean. This apparent agreement seems at first to suggest a simplification of the theory of the values of $h$ and $k$, and that they might be calculated on an equilibrium theory of the tides and so lead to a fairly accurate determination of the Earth's rigidity. But Schweydar's recent investigations show that this is not so, and that theoretically the matter is one of great complexity.

We turn for a little to the theoretical side which we owe mainly to Lord Kelvin. The matter was one of life-long interest to him, and the investigations (Thomson and Tait, "Natural Philosophy") form the basis of most subsequent 
calculations. The quantities $h$ and $k$ are not independent, but are related and dependent on the physical properties of the Earth as a whole. The simplest assumption that can be made is to regard the Earth as a uniform sphere which is incompressible, but possesses rigidity $\mu$, and further that the tides may be computed on an equilibrium theory. We then find that

$$
k=\frac{3}{5} h, \text { and } h=\frac{5}{2} /\left(\mathrm{I}+\frac{\mathrm{I} 9 \mu}{2 g \rho a}\right) .
$$

Thus if we accept the experimental value $h-k=\mathrm{I} / 3$ we get $h=5 / 6$ and $k=\mathrm{I} / 2$ while $\mu=7^{\circ} \mathrm{I} \times \mathrm{IO}^{11}$ dynes per sq. $\mathrm{cm}$. This value of $\mu$ which is nearly that of steel, formed the ground of Kelvin's estimate of the Earth's rigidity. Darwin, however, did not accept this, but regarded the observed reduction of the fortnightly tides as due to the difference between the dynamical and the equilibrium theory (cf. Lamb, "Hydrodynamics").

The preceding result, however, conflicts with data derived from the free period of precessional nutation of the Earth as derived from astronomical observations. Larmor ("Proc. R. S.," Vol. 82, p. 89, I909) shows that

$$
k=\left(\mathrm{I}-\frac{\mathrm{T}_{0}}{\mathrm{~T}}\right)\left(\frac{2 \varepsilon g}{\omega^{2} a}-\mathrm{I}\right)
$$

where $T_{0}$ is the theoretical Eulerian period 306 days,

$T$ the observed Chandler's period 428 days,

$\omega$ the angular velocity of rotation of the Earth.

and $\epsilon$ is the ellipticity of the ocean surface. Thus since $\omega^{2} a / g=\mathrm{I} / 289$ and $\epsilon$ has practically the same value, we get $k=0.28$, and this with $h-k=0.33$ gives $h=0.6 \mathrm{I}$ which does not satisfy the relation $k=\frac{3}{5} h$ and leads to a higher estimate of the Earth's rigidity.

Schweydar ("Veröff. Kön. Preuss. Geod. Instit.," No. 54, I 9I2) investigates the reason for the discrepancies. He takes account of the oceanic tides, and further introduces Wiechert's assumption that the solid part of the Earth consists of a shell of density 3.2 and thickness I $500 \mathrm{~km}$., and a nucleus of density $8 \cdot 2$. It would perhaps have been an advantage to have 
treated the two separately. His main point is, however, that while the corporeal tides may be computed at their equilibrium values, the oceanic tides must be considered dynamically. The differences of Hecker's results in the $\mathrm{N}-\mathrm{S}$ and $\mathrm{E}-\mathrm{W}$ directions are attributed to the unequal action of the oceanic tides in different directions, while $h$ and $k$ are substantially changed from what they would be on the simple theory, by terms depending on the oceanic tides. On certain assumptions with regard to the depth of the ocean he finds that the general rigidity of the earth may be from two to three times that of steel, and that the results obtained from the semi-diurnal lunar terms may thus be brought into accordance with the astronomical data.

He concludes that the semi-diurnal lunar terms indicated by seismographs are not of much real value in determining the value of the earth's rigidity.

We may remark in passing that somewhat similar numerical results would follow by taking account of the Earth's compressibility for one of the most important points obtained by Love ("Problems of Geophysics") is that the compressibility would substantially increase the estimated value of $h$ without much affecting $k$, so that the experimental values when corrected for compressibility would lead to improved concordance and to higher values of the rigidity.

Schweydar's next step is to argue that the nearly diurnal lunar declination tide due to the potential

$\mathrm{O}_{2}=\frac{3}{4} m g \frac{a^{2} r^{2}}{c^{3}}\left(\mathrm{I}-\frac{5}{2} e^{2}\right) \sin w \cos ^{2} \frac{1}{2} w \sin 2 \phi \cos \left(t+\lambda-t_{0}\right)$

is better adapted to give the value of $h-k$, because on a certain assumption as regards the depth of the ocean (which is not the same as that made in the discussion of the semi-diurnal term) the effect of the oceanic tides may be neglected.

He gives the following results obtained at Freiberg i.S. with pendulums in azimuth $35^{\circ} \mathrm{E}$ of $\mathrm{N}$ and $55^{\circ} \mathrm{E}$ of $\mathrm{S}$.

Pendulum I.

Observed. $0^{\prime \prime} \cdot 004 \mathrm{I} 2 \cos \left(t-273^{\circ}\right)$. Computed. $\mathrm{o}^{\prime \prime} \cdot 00493\left(\cos t-280^{\circ}\right)$. Pendulum II.

Observed. $0^{\prime \prime} \cdot 003$ I8 $\cos \left(t-248^{\circ}\right)$. Computed. $0^{\prime \prime} \cdot 00363(\cos t-249)$. 
This leads to $(\mathrm{I}-h+k)=0.85$ or $h-k=0.15$ and along with $k=\frac{3}{5} h$ this gives $h=0.38$ leading to a general rigidity about three times that of steel. This, however, neglects the influence of compressibility.

As a whole the position with regard to earth tides as indicated by movements of seismograph zero is rather unsatisfactory. The doubt that may very reasonably be entertained as to purely instrumental sources of error in the observations, renders theoretical discussion somewhat futile until we know exactly what the facts are.

It is gratifying to know that the International Seismological Association has in view experiments with horizontal pendulums at different points of the Earth, which ought to throw much light on the phenomena. It would also be useful if other means of experimenting could be devised. A solid surface undergoes, as we have seen, tilting of amount

$$
\frac{\mathrm{I}}{g} h \frac{\partial \mathrm{W}_{2}}{\partial x} \text {. }
$$

This must result in an apparent change of position of any star. But the effect is so small that it is hardly likely to be detected by astronomical means. On the other hand a liquid surface undergoes tilting of amount

$$
\frac{\mathrm{I}}{g}(\mathrm{I}+k) \frac{\partial \mathrm{W}_{2}}{\partial x} \text {. }
$$

The differential tilting between a liquid surface and a solid surface beneath it is

$$
\frac{\mathrm{I}}{g}(\mathrm{I}-h+k) \frac{\partial \mathrm{W}_{2}}{\partial x} .
$$

The suggestion I wish to make is that such an arrangement would show interference fringes parallel to the line of no resultant tilt, and that the direction would thus change in the course of the day. It might be practicable in this way to study the operation of the variable tilt

$$
\frac{\mathrm{I}}{g}(\mathrm{I}-h+k) \frac{\partial \mathrm{W}_{2}}{\partial x}
$$




\section{CHAPTER X.}

\section{STATISTICAL.}

PERHAPS one of the most striking features revealed by the systematic observations of earthquakes is the rarge number detected by seismographs as compared with those earthquakes which obtain notoriety in the public press. This is owing to the fact that a large number of earthquakes are of but small intensity, while of the large earthquakes or megaseisms the majority fortunately occur at the bottom of the sea or in unpopulated regions without causing loss of human life.

Earthquakes whether large or small are of interest to the seismologist.

The number recorded at any given station depends on the position of the station, as well as the sensitiveness of the instruments. As illustrating the number recorded in a nonseismic region I give the numbers recorded at Eskdalemuir on the Galitzin Seismographs in I9I I.

$\begin{array}{cccccccccccr}\text { Jan. } & \text { Feb. } & \text { Mar. } & \text { Apr. } & \text { May } & \text { June } & \text { Jul. } & \text { Aug. } & \text { Sept. } & \text { Oct. } & \text { Nov. } & \text { Dec. } \\ \text { I6 } & \text { 10 } & 8 & \text { I9 } & \text { 19 } & 20 & 24 & 23 & 28 & 32 & \text { 16 } & 20\end{array}$

The total for the year is 235. Most of these were small, but sixteen at least deserved to be called megaseismic. In particular the earthquake of 3 January which occurred in Turkestan $\left(4 \mathrm{I}^{\circ} \mathrm{N} \quad 77^{\circ} \mathrm{E}\right.$ ) was so violent that the seismographs at Pulkowa were broken, and even at Eskdalemuir the needle of one of the galvanometers was thrown out of action.

I ought perhaps to say that none of the above earthquakes were of local character. I was never able at 'Eskdalemuir to detect any indication of earthquakes reported to have taken place in Perthshire, and even the Glasgow earthquake of December, I9I0, which caused considerable public excitement 
there, produced no perceptible effect on the Eskdalemuir seismographs.

It is now the custom for observatories to exchange bulletins, and for many years Milne has published ("British Assoc. Reports") annual tables of data from all sources. An annual table is now also issued under the auspices of the International Association of Seismology (Strassburg). The importance of such bulletins and tables can hardly be overrated.

They enable one to confirm or correct inferences and greatly extend our knowledge of the number of earthquakes which occur at all points of the earth. Milne estimates that the annual output from all sources is nearly 60,000 earthquakes.

It has long been noted that the seismograms obtained at a given station show an extraordinary similarity for separate earthquakes that occur in the same region of the Earth. In some cases the seismograms might almost be superposed. This is a matter deserving careful investigation as it points not perhaps so much to a difference of the properties of the interior of Earth in different directions, as to a characteristic origin of the earthquakes occurring in one and the same region.

While there is a general agreement that an earthquake is caused by a rupture of the rocks within the earth's crust, we have no very definite knowledge as to the primary cause of the rupture. It is not unnatural to look for such a cause in the tidal stresses of solar and lunar origin. In particular we might look for a preponderance of the number of earthquakes at the times of syzygy of Sun, Earth, and Moon. Such investigations have been made but do not appear to result in clear evidence of such an association (Milne, "Earthquakes," p. 250). Another way of dealing with the occurrence of earthquakes, and which is well known in connexion with the analysis of meteorological and magnetic data, is to express the observations by a Fourier series in terms of the time, either solar or lunar. Such investigations have been made by Knott ("Proc. R.S.," I 897) and by Davison ("Phil. Trans. A.," Vol. I84, I 893). These have been critically examined by Schuster ("Proc. R.S.," I 897).

A question arises as to what should be included in the data submitted to analysis. It is known that a large earthquake is 
followed by a large number of minor shocks, and the point is whether these minor shocks should be treated as separate quakes or regarded as part of the primary shock. Again, ought there to be a classification according to intensity? I should doubt if agreement of opinion could be reached $a$ priori. It seems to me to rest with the investigator to decide whether he shall classify and group or not, but it then rests with him to show that he reaches a conclusion which is a real contribution to knowledge.

There is a growing doubt whether a Fourier analysis of an observational quantity is really the best way of expressing results with a view to physical explanation of the cause, but however that may be, we must agree with Schuster that there is a right and a wrong way of making the Fourier analysis, and that the right way is to take the data as they stand and not to apply any preliminary smoothing process. It appears to me that if a smoothing process was permissible it would, carried to excess, be an argument for never making observations at all.

It is not sufficient to compute the Fourier co-efficients. We have to show that any term so obtained is substantially greater than what might be expected as the result of fortuitous occurrence. The criterion given by Schuster is as follows :-

"If a number $n$ of disconnected events occur within an interval of time $\mathrm{T}$, all times being equally probable for each event, and if the frequency of occurrence of these events is expressed in a series of the form

$$
a\left\{\mathrm{I}+\rho_{1} \cos 2 \pi \frac{\left(t-t_{1}\right)}{\mathrm{T}}+\ldots \rho_{m} \cos 2 m \pi \frac{(t-t)}{\mathrm{T}}\right\}
$$

the probability that any of the quantities $\rho$ has a value lying between $\rho$ and $\rho+\delta \rho$ is

$$
\frac{n}{2} \rho \delta \rho e^{-n \rho^{2} / 4}
$$

and the 'expectancy' for $\rho$ is

$$
\sqrt{\pi / n} \text {." }
$$

On this basis Schuster finds that the lunar terms obtained by Knott must be discarded, but on the other hand the annua! 
periodicity with a maximum in winter and the diurnal periodicity with a maximum about noon obtained by Davison from earthquake statistics may be regarded as fairly well established.

Although the small table at the beginning of this chapter is too limited to justify any general conclusion it will serve to illustrate the application of Schuster's method.

I find that the Fourier expression is given with sufficient accuracy by

$$
\mathrm{N}=2 \mathrm{O}\left(\mathrm{I}+0_{4} 4 \cos \overline{t+\mathrm{I} 20^{\circ}}+\mathrm{O}^{\circ} \mathrm{I} \cos \overline{2 t+\mathrm{I} 20^{\circ}}\right)
$$

where $t$ is the time reckoned from I January at the rate of $30^{\circ}$ per month.

The expentancy is $\sqrt{\pi / 235}$ or 0.12 , and we should thus argue that the semi-annual term is worthless while the annual term with its maximum at the end of August is important.

The practical application of Fourier analysis to observational quantities is really very simple, and since it does not usually find a place in physical textbooks, a few remarks about it may not be out of place here.

If the observed quantity $f$ is to be expressed by means of a Fourier series

$$
f=\frac{1}{2} a_{0}+\sum_{n=1}^{n=\infty}\left(a_{1} \cos n \theta+b_{1} \sin n \theta\right)
$$

between the limits o and $\mathrm{T}$ where $\theta=2 \pi t / \mathrm{T}$, we have

$$
\begin{aligned}
a_{0} \mathrm{~T} & =\int_{0}^{\mathrm{T}} f(t) d t \\
\frac{1}{2} a_{n} \mathrm{~T} & =\int_{0}^{\mathrm{T}} f(t) \cos \frac{2 \pi n t}{\mathrm{~T}} d t \\
\frac{1}{2} b_{n} \mathrm{~T} & =\int_{0}^{\mathrm{T}} f(t) \sin \frac{2 \pi n t}{\mathrm{~T}} d t .
\end{aligned}
$$

If $f(0)=f(T)$ then no difficulty occurs; but if, as generally happens with observed quantities, $f(0) \neq f(\mathrm{~T})$ then the function $f$ is not strictly periodic in time $\mathrm{T}$, and this at once sets a limit. The series represents the function $f$ between the limits but not at the limits, for the series then gives $\frac{1}{2}\{f(0)+f(\mathrm{~T})\}$ at the limits.

This difficulty is often dealt with in practice by assuming that the difference $f(0)-f(T)$ is incident linearly, during the interval $\mathrm{T}$ and it is subtracted from $f$ before analysing. This 
merely confuses the issue, and it is less objectionable to take the function $f$ as observed and to remember that in so far as $f(\mathrm{o})$ differs from $f(\mathrm{~T})$ the representation is incomplete.

The data are, however, usually presented in the form of hourly values in solar time or lunar time according to the source we have reason to suspect as contributing to the effect. If then $f_{0}, f_{1} \ldots f_{24}$ represent the values of $f$ for the various hours the formula become

$$
\begin{gathered}
24 a_{0}=\frac{1}{2}\left(f_{0}+f_{24}\right)+\sum_{m=\mathrm{I}}^{m=23} f_{m} \\
\mathrm{I} 2 a_{n}=\frac{1}{2}\left(f_{0}+f_{24}\right)+\sum_{m=\mathrm{I}}^{m=23} f_{m} \cos \left(m n \mathrm{I} 5^{\circ}\right) \\
\text { I } 2 b_{n}=\sum_{m=\mathrm{I}}^{m=23} f_{m} \sin \left(m n \mathrm{I} 5^{\circ}\right) .
\end{gathered}
$$

The numerical process is simple since the terms collect into groups with the same numerical coefficients.

In these expressions $f_{n n}$ may be the actual value at the hour $m$ or the mean for an hour centering at $m$. Neither is strictly correct for an infinite Fourier series although the former is correct for a limited series, ending with $n=24$. Here again the representation is incomplete when $f_{0} \neq f_{24}$.

Unless the quantity $f$ varies in a very regular manner, one day's observations would not be sufficient, and the hourly values are then averaged for say a month. A similar process would then be applied to the coefficients so obtained to determine their annual periodicity.

This method, however, fails unless the day or the year are real periods of the phenomena; and may, as we have seen, give a false impression of periodicity unless Schuster's criterion can be applied.

The only general method of detecting periodicity is due to Schuster. I quote from his paper ("Proc. R. S.," l.c.). "Let $y$ be a function of $t$, such that its values are regulated by some law of probability, not necessarily the exponential one, but acting in such a manner that if a large number of values of $t$ be chosen at random there will always be a definite fraction of that number depending on $t_{1}$ only, which lie between $t_{1}$ and $t_{1}+T$, where $T$ is any given time interval. 
"Writing

$$
\mathrm{A}=\int_{t_{1}}^{t^{1}+\mathrm{T}} y^{\prime} \cos k t d t \text { and } \mathrm{B}=\int_{t_{1}}^{t_{1}+\mathrm{T}} y \sin k t d t
$$

and forming $\mathrm{R}=\left(\mathrm{A}^{2}+\mathrm{B}^{2}\right)^{\frac{2}{2}}$

the quantity $R$ will, with increasing values of $T$, fluctuate about some mean value, which increases proportionally to $T^{\frac{1}{2}}$, provided $\mathrm{T}$ is taken sufficiently large.

"If this theorem is taken in conjunction with the two following well-known propositions:-

"( I) If $y=\cos k t, \mathrm{R}$ will, apart from periodical terms increase proportionally to $\mathrm{T}$.

"(2) If $y=\cos \lambda t, \lambda$ being different from $k$, the quantity $\mathrm{R}$ will fluctuate about a constant value,

it is seen that we have means at our disposal to separate any true periodicity of a variable from among its irregular changes, provided we can extend the time limits sufficiently."

The method of applying this will be found in "Camb. Phil. Trans.," Vol. I8, I900.

I have referred to this problem specially, because statistics about earthquakes are rapidly increasing in number and accuracy, and the search for periodicity will again be taken up. It is desirable that the search should proceed on the lines indicated by Schuster.

I understand that by application of this method, Prof. Turner ("Brit. Assoc.," I9I2) finds evidence of a 452 day period of earthquake activity. The result is interesting as it is so near the Chandler period of precessional nutation, and here we may fitly close the volume with a quotation from Milne ("Earthquakes," 6th edition, 1913, p. 377): "Speaking generally, so far as I know, neither tidal, barometric, thermometric, solar, lunar, or other epigene influences beyond those mentioned, show a relationship to the periodicity or frequency of megaseismic activity. Their frequency is apparently governed by activities of hypogene origin." 


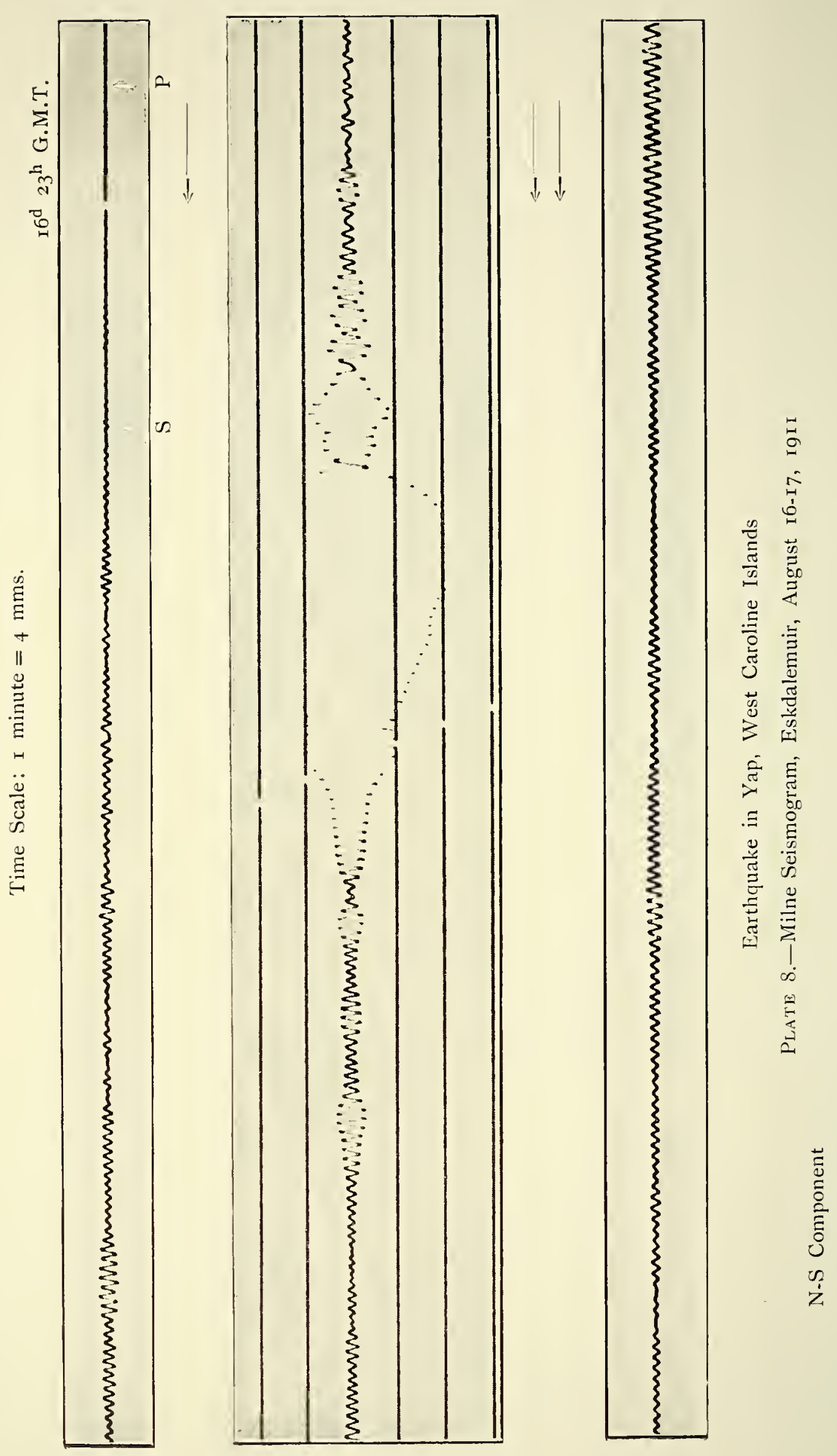


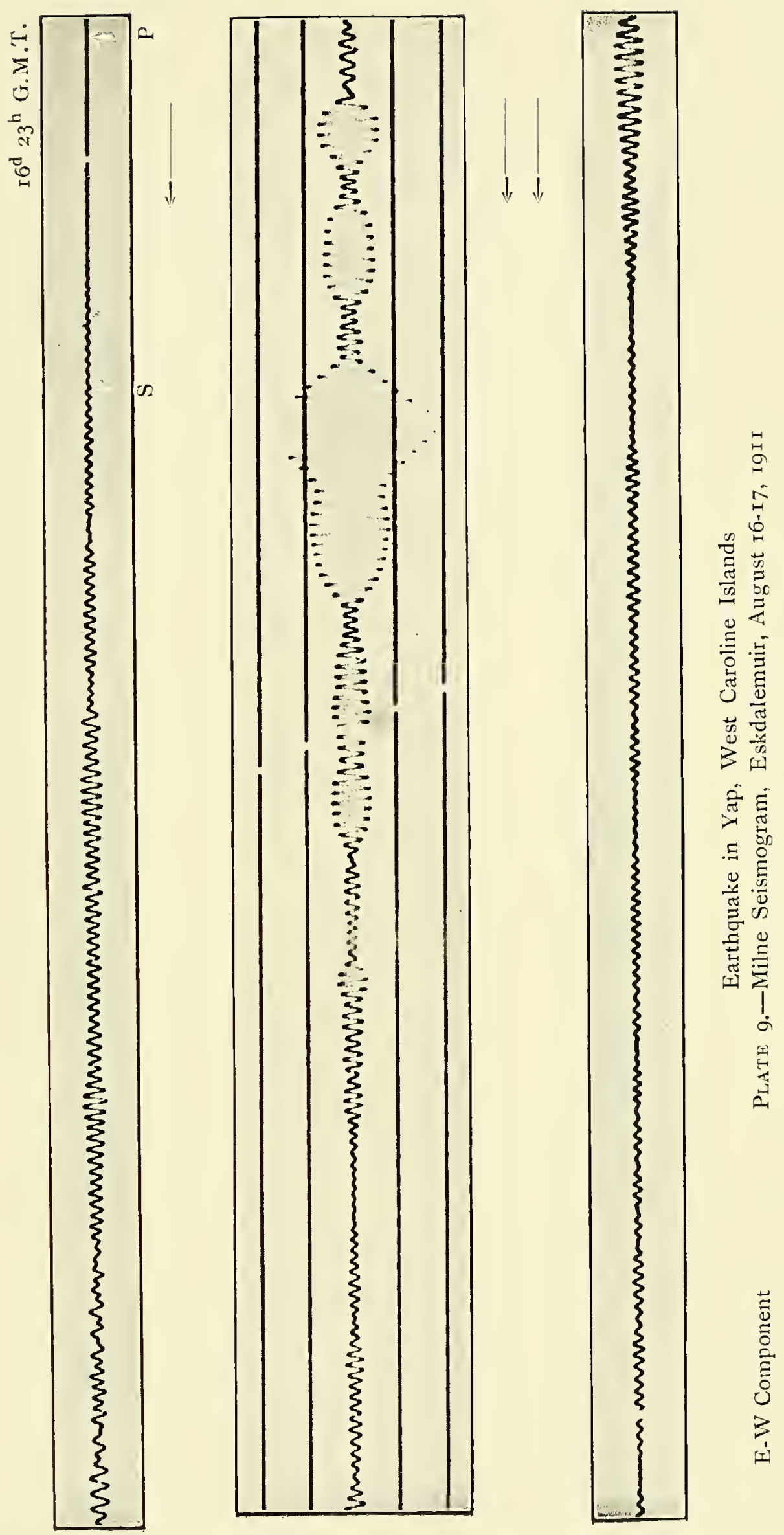





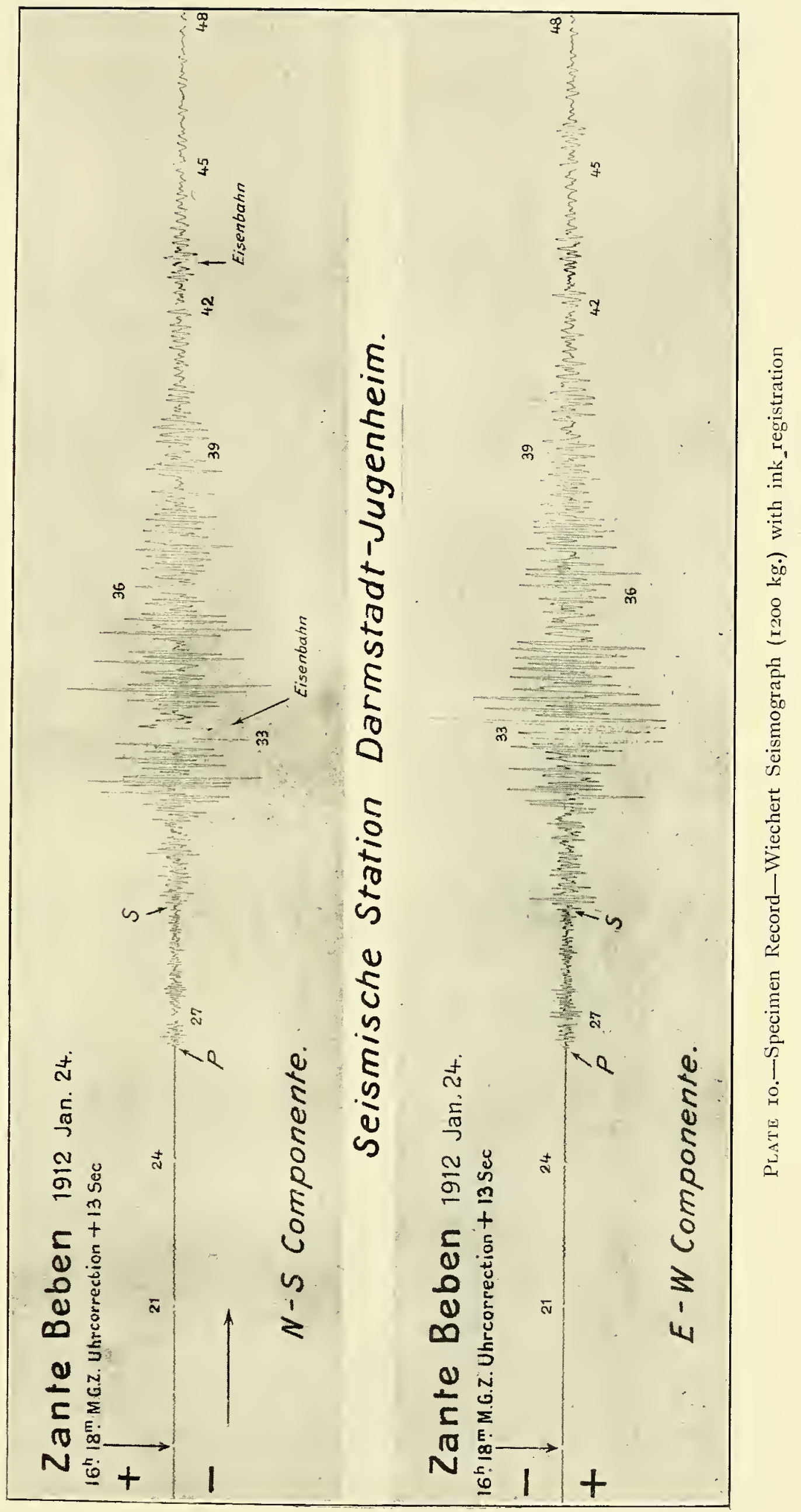








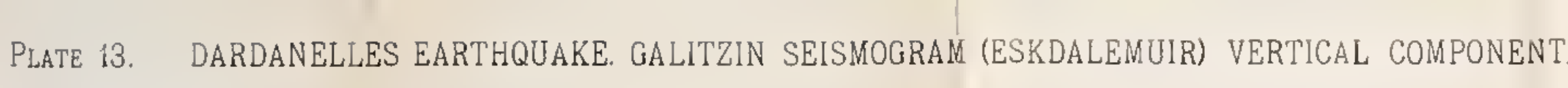

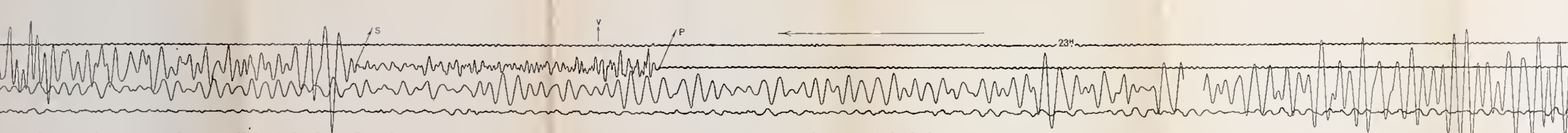

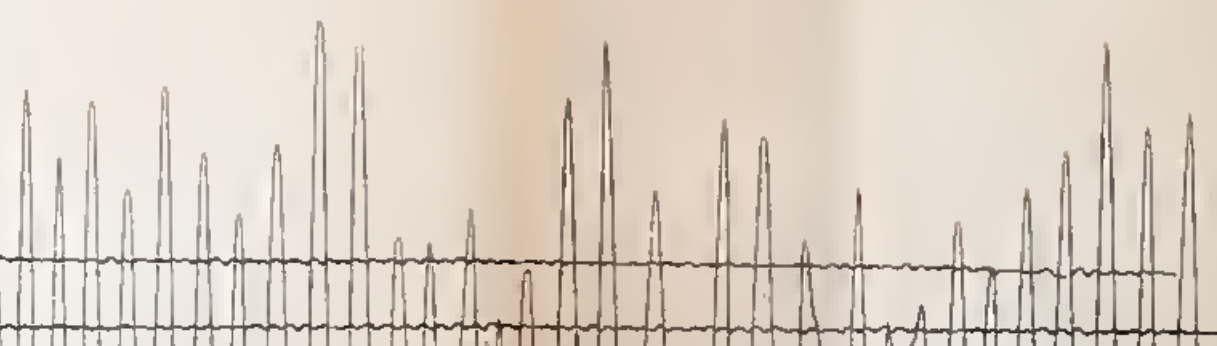



40

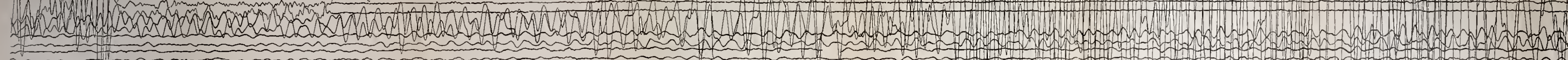
1 (n) 
SMITHSONIAN INSTITUTION LIBRARIES

39088 00225604 8

nmah QE634.W3X

Modern seismology. 